\title{
Post-stroke depression : the need for a biopsychosocial approach
}

Citation for published version (APA):

Aben, I. J. J. (2004). Post-stroke depression : the need for a biopsychosocial approach. [Doctoral Thesis, Maastricht University]. Universiteit Maastricht. https://doi.org/10.26481/dis.20041015ia

Document status and date:

Published: 01/01/2004

DOI:

10.26481/dis.20041015ia

Document Version:

Publisher's PDF, also known as Version of record

\section{Please check the document version of this publication:}

- A submitted manuscript is the version of the article upon submission and before peer-review. There can be important differences between the submitted version and the official published version of record.

People interested in the research are advised to contact the author for the final version of the publication, or visit the DOI to the publisher's website.

- The final author version and the galley proof are versions of the publication after peer review.

- The final published version features the final layout of the paper including the volume, issue and page numbers.

Link to publication

\footnotetext{
General rights rights.

- You may freely distribute the URL identifying the publication in the public portal. please follow below link for the End User Agreement:

www.umlib.nl/taverne-license

Take down policy

If you believe that this document breaches copyright please contact us at:

repository@maastrichtuniversity.nl

providing details and we will investigate your claim.
}

Copyright and moral rights for the publications made accessible in the public portal are retained by the authors and/or other copyright owners and it is a condition of accessing publications that users recognise and abide by the legal requirements associated with these

- Users may download and print one copy of any publication from the public portal for the purpose of private study or research.

- You may not further distribute the material or use it for any profit-making activity or commercial gain

If the publication is distributed under the terms of Article $25 \mathrm{fa}$ of the Dutch Copyright Act, indicated by the "Taverne" license above, 


\section{POST-STROKE DEPRESSION}

THE NEED FOR A BIOPSYCHOSOCIAL APPROACH 
De aarde gaat voort, zichzelf desondanks; zo moe -

alles aan me is moe.

\section{Waraku}

Cover drawing: by Paul Jorritsma, from 'Met de blik op oneindig, berichten uit het Printed by: bejaardenhuis'; Arnhem, Ampersand BV, 1998.

ISBN 90-5278-424-8

(C) 2004 IJJ Aben, Maastricht

All rights are reserved, whether the whole or part of the material is concerned. No part of this publication may be reproduced, stored in a retrieval system, or transmitted in any form or by any means, electronic, mechanical, photocopying, recording, or otherwise, without written permission from the copyright owner. 


\title{
POST-STROKE DEPRESSION
}

\author{
THE NEED FOR A BIOPSYCHOSOCIAL APPROACH
}

\section{PROEFSCHRIFT}

Ter verktrijging van de gxaad van doctor aan de Universiteit Maastricht, op gezag van de Rector Magnificus,

Prof. mr. G.P.M.F. Mols

volgens het besluit van het College van Decanen, in het openbaar te verdedigen

op vrijdag 15 oktober 2004 om 12:00 uur

door

Ivo Juliën Joseph Aben

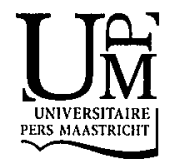




\section{Promotor}

Prof. dr. F.R.J.Verhey

\section{COPROMOTOR}

Dr. A. Honig

\section{BEOORDELINGSCOMMISSIE}

Prof. dr. J. van Os (voorzitter)

Prof. dr. Th. Heeren (Universiteit Utrecht)

Prof. dr. C. Jonker (Vrije Universiteit Amsterdam)

Prof. dr. M. Limburg

Prof. dr. H. Merckelbach

The research described in this thesis was carried out at the Maastricht Brain and Behaviour Institute and both the department of Neurology and Psychiatry \& Neuropsychology, Maastricht University, Maastricht, the Netherlands, in collaboration with the University Hospital Maastricht.

Financial support for the publication of this thesis has been kindly provided by Janssen-Cilag BV, Bristol-Myers Squibb BV, Eli Lilly Nederland BV, and AstraZeneca BV. 


\section{CONTENTS}

\section{Chapter 1}

Introduction

CHAPTER 2

Research into the specificity of depression after stroke: a review of an unresolved issue

\section{CHAPTER 3}

Validity of the Beck Depression Inventory, Hospital Anxiety and Depression Scale, SCL-90, and Hamilton Depression Rating Scale as screening instruments for depression in stroke patients

\section{CHAPTER 4}

A comparative study into the one year cumulative incidence of depression after stroke and inyocardial infarction

\section{Chapter 5}

Personality and vulnerability to depression in stroke patients: a 1-year prospective follow-up study

\section{Chapter 6}

Focal or generalised vascular brain damage and vulnerability to depression after stroke: a 1 -year prospective follow-up study

\section{CHAPTER 7}

Risk factors for post-stroke depression: a two-step Cox regression analysis

\section{CHAPTER 8}

Recognition and treatment of post-stroke depression in general practise

CHAPTER 9

General discussion

REFERENCES

DANKWOORD 



\section{CHAPTER 1}

Introduction

Al roeiend gaan wij door nevels heen, daarachter

ligt breeduit de zee.

Shiki 


\section{INTRODUCTION}

\section{DEFINING THE PROBLEM}

From a Health Care perspective, stroke is a major health problem in the West. According to the Dutch Heart Foundation, every year about 30,000 people suffer a stroke in the Netherlands and, at present about 120,000 people are estimated to live with the consequences of stroke. Of those stroke survivors, about $70 \%$ have cognitive, emotional and/or behavioural problems, while about $40 \%$ experience disabilities in every day functioning. This makes stroke the most important cause of handicap in the Netherlands, which costs society about two billion euro a year. ${ }^{1}$

In recognition of the enormity of the medical problem of stroke, specialised stroke units and services have been developed throughout the country and their numbers are rapidly increasing. In 1999, there were 59 recognised stroke services, while another 80 health care facilities were considering starting a stroke service in their region. ${ }^{2}$

Depression has been found to be the most frequent psychiatric disorder in stroke patients. Not only does it cause serious suffering in the patient but evidence also suggests that it has a negative effect on stroke rehabilitation outcome, which subsequently contributes to higher health care consumption and costs. Furthermore, post-stroke depression (PSD) has been shown to have a negative effect on life expectancy. Despite the clinical importance of PSD, it stays frequently unrecognised and untreated. ${ }^{3}$

To improve the quality of life of stroke survivors, PSD should be diagnosed at an early stage to allow treatment interventions. Knowledge about the epidemiology of PSD may help to reach this goal by gaining insight in the incidence, phenomenology and risk factor profile.

From a scientific point of view, damage to circumscriptive parts of the brain has prompted neurobehavioral researchers throughout history to learn more about the topographic organisation of human behaviour. ${ }^{4}$ Stroke is the commonest cause of such focal brain damage and it was recognised by Gainotti (1969) that some stroke patients showed intriguing specific psychiatric phenomena such as indifferent and catastrophic reactions. ${ }^{5}$ As stated above, disturbed mood, especially depression, has been shown to be the most frequent behavioural disorder after stroke. ${ }^{6}$ It may not be surprising that theories have been developed about the cause of this, so-called post-stroke depression in relation to the location of the stroke lesion. Gainotti described differential effects on emotional behaviour after right- versus left hemisphere lesions and built a theory on the lateralised organisation of emotions. ${ }^{7,8}$ Robinson was the first to attribute the majority of cases of PSD to left frontal strokes.? This initial finding has stimulated research in this field to a great extent during the last two decades. However, the lesion location hypothesis of PSD has 
not found unequivocal support through research findings and, therefore, remains controversial. ${ }^{10}$

Seen from a different perspective, stroke can be considered an unexpected stroke of misfortune, which, like every other negative event in life, challenges a person to adapt to his situation adequately in order to maintain or restore his balance in life. Stroke can have a devastating impact on a person's life. Physically, one may be confronted with a sudden loss of basic abilities such as walking or talking and a variety of other neurological handicaps. "These may be accompanied by aspecific complaints, such as lack of energy and loss of appetite. Psychologically, severe cognitive problems such as memory, speech or attention deficits may arise. Moreover, emotionally one may experience less stability or even pathological phenomena such as pathological crying. On a social level, the patient may lose one's usual social role, such as being a caring parent or spouse, or a productive and respected colleague. In severe cases, patients may permanently become dependant on others.

From this point of view, it is easy to understand that PSD occurs with high frequency. However, research has predominantly focused on biological explanations (especially the lesion location hypothesis) for the development of post-stroke depression.

This thesis attempts to present the case for more emphasis on the integration of a biopsychosocial model of PSD alongside the current predominantly biological approach.

\section{POST-STROKE PSYCHOPATHOLOGY}

Up to $70 \%$ of stroke patients suffer from cognitive, emotional, or behavioural problems. ${ }^{12}$ As stated above, some of these problems are believed to be direct consequences of damage to brain tissue that is involved in the organisation of mental functioning. Although there is a growing tendency to explain brain behaviour relationships in terms of disrupted neuronal networks, the traditional localisation view is still evident for certain cognitive functions. For instance, aphasia can be closely related to damage of the speech centres of Broca or Wernicke, and phenomena as anosognosia (i.e. loss of the ability to recognise one's own illness), apraxia (i.e. loss of the ability to carry out familiar, purposeful movements in the absence of paralysis or other motor or sensory impairment), or acalculy (i.e. loss of the ability to calculate) can result from damage to the left parietal lobe. ${ }^{11}$

Several other phenomena of disturbed behaviour have been suggested to be specific consequences of brain lesions. The catastrophic and indifferent reaction, emotional lability (better referred to as emotionalism), and aprosody are examples of such phenomena. ${ }^{6}$ The catastrophic reaction concerns an acute refusal reaction 
with strong emotions of anger or despair in response to confrontation with a task that has become difficult due to the consequences of stroke. The indifferent reaction, on the contrary, refers to an indifferent attitude towards one's disabilities and handicaps, while being aware of them. This is notably different from anosognosia in which the patient does not recognise that he or she is disabled. In emotionalism, a patient has less control over when they begin to start crying or laughing. This may happen without a subjective experience of feeling sad or happy, and the patient cannot control his or her emotional response as he or she used to in social situations. Aprosody is the inability to either produce or comprehend the affective components of speech or gesture. In expressive aprosody, a patient is unable to intone affect into his or her speech by variations in pitch, thythm, and stress of pronunciation. In receptive forms, one cannot understand the prosodic information communicated by others anymore Although aprosody is associated with right-hemisphere damage, no clear evidence of specific lesion locations has been found to explain the different forms of this phenomenon.

Other psychopathological conditions are not exclusive sequels of stroke but do also occur in patients without detectable brain damage, such as anxiety disorders, affective disorders and dementia. Nevertheless, these conditions have also been studied in relation to vascular brain damage. Vascular dementia is, after Alzheimer's disease the most common type of dementia and is believed to result rather from one strategic localised infarct (e.g. thalamic) or from multiple small ones (multi infarct dementia). ${ }^{13-15}$ Generalised Anxiety Disorder (GAD) has also been suggested to be specifically induced by brain damage. Studies suggested that GAD occurs in $25-30 \%$ of stroke patients and that GAD with comorbid depression is associated with left-hemisphere lesions while GAD alone is associated with tighthemisphere lesions. ${ }^{16,17}$

\section{PATHOGENIC THEORIES Of PSD}

Last but not least, affective disorders have received much attention in this type of neuropsychiatric research since Robinson first presented his lateralisation findings concerning PSD. The lateralisation phenomenon was explained by the idea that negative emotions are processed in the left hemisphere, whereas positive emotions are processed in the right hemisphere. ${ }^{7}$ According to this model, mania has been proposed to be more frequent in right hemisphere lesions, whereas depression has been related to the left hemisphere. ${ }^{18}$ Alternatively, some evidence suggested lateralised effects of stroke on the availability of serotonin receptors. Stroke in the right hemisphere was shown to induce upregulation of serotonin receptors in the injured hemisphere while this failed to occur after left sided stroke. Moreover, a negative correlation was demonstrated between the severity of depression and the ratio between ipsilateral to contralateral serotonin receptor density. ${ }^{19}$ 
Furthermore, PSD was shown to occur more frequently in stroke patients in whom the frontal region of the brain was damaged. The association between PSD and frontally located lesions was suggested to be explained by the extent to which these lesions damage neurotransmitter systems involved in mood regulation. The serotonergic and noradrenergic neural pathways that connect the brainstem with almost the entire cortex are made up of long axons which are directed towards the frontal lobe and, from there, bend round and spread to all other brain areas. So, the more a stroke lesion is located to the frontal pole the more these serotonergic or noradrenergic pathways would be disrupted. ${ }^{20}$

Recently, Vataja et al reported that PSD patients had a higher number and latger volume of infarcts affecting the prefrontosubcortical circuits, especially the caudate, pallidum, and genu of the internal capsule, with left-sided predominance. This suggests that damage to connections between the limbic system and the forebrain, which are involved in emotional behaviour, make stroke patients more vulnerable to depression. ${ }^{21}$

A number of studies from independent research groups were able to replicate the lesion location findings but, unfortunately, the opposite was also true: at present, three meta-analyses that systematically evaluated all available evidence on this topic, concluded that, overall, there is no support for left hemisphere strokes to be more prone to induce depression than right hemisphere lesions. ${ }^{22-24}$

In connection with the concept of vascular dementia, the role of (generalised) vascular brain damage in the pathogenesis of depression in the elderly caught the attention of researchers. Krishnan and co-workers observed that elderly depressed patients often had no personal and family history of depression but they shared a history of cardiovascular disease as well as signs of vascular damage on MRI scans. Subsequently, they introduced the term vascular depression. Krishnan et al. showed that vascular depression (as defined by a diagnosis of depression in combination with the presence of white matter hyperintensities on an MRI scan) is related to older age with late age at onset of depression, and non-psychotic subtype of depression. ${ }^{25} \mathrm{~A}$ relationship between vascular damage measured as subcortical and periventricular white matter lesions on MRI scans and depressive symptomatology has also been established in community based research. ${ }^{26}$. Thus, in addition to focal brain damage, generalised vascular damage may be an alternative neurobiological factor that is involved in the pathogenesis of PSD.

\section{RATIONALE OF THIS THESIS}

Despite the numerous studies that have been published concerning PSD, many questions about the occurence, phenomenology, and pathogenesis of PSD are still unanswered due to inconsistent research findings. Most probably, the explanation for this lies in a number of methodological difficulties that PSD researchers are 
facing. How can PSD be diagnosed reliably, especially in patients with disabilities such as aphasia or anosognosia? How does one differentiate PSD from 'physiological' emotional distress which is part of one's functional coping mechanisms? Is depression that occurs a few weeks after stroke comparable to depression after 1 or 2 years? These and other methodological difficulties will be described in more detail in chapter 2.

Another explanation for the contradictory research findings may hide in the relative neglect of psychological and social factors which might contribute to the development of PSD and might even interact with the biological vulnerability caused by damage to specific brain areas involved in the regulation of emotions. The strong influence of the lesion location hypothesis of Robinson on PSD research during the last decades has discredited the plausibility of the reactive depression hypothesis. In fact, combining the two models may result in a more holistic model that explains the huge variance in the findings that research has yielded.

As a consequence of the uncertainties discussed above, there is still debate on how frequently PSD occurs, which symptoms are most discriminative in recognizing the syndrome, and which factors play a role in its development.

It is important to gain insight in these topics, not only because it is a great burden to the patient and his or her relatives but also because PSD has been found to prolong hospital admissions, negatively interfere with rehabilitation programmes and shorten life expectancy. ${ }^{27,} 28$ This costs our Dutch society millions of euros a year and this will probably rise as a consequence of the aging population and the increase in stroke survival.

This thesis is an attempt to contribute to our insight into post-stroke depression by studying its incidence and a number of potential risk factors. In doing so, it is tried to overcome some of the methodological difficulties in order to improve the reliability of our findings as compared to earlier studies on PSD. This has been done by selecting patients from a hospital with a regional care function, measuring depression with a structured clinical interview and using a sensitive screening instrument during a 1-year follow up period. Furthermore, several potential risk factors were studied enabling multivariate analysis strategies (including survival analysis) to study the biological and psychological theories of PSD in one single cohort. During the study, the quality of data collection was monitored carefully in order to maximize the completeness of the follow up and to enable evaluation of the effects of participation refusal and attrition during follow-up. The quality of depression screening was evaluated after a one-month period. 


\section{AIMS AND OUTLINE OF THIS THESIS}

The central issue of this thesis is to study the interaction between biological, psychological, and social factors in patients with PSD. In doing so, the relative contribution is clarified, and the nature of this interaction is explored.

\section{What is currently known about PSD?}

In a review article, we explored the methodological difficulties that may underlie the inconsistencies in research findings about PSD (chapter 2). Furthermore, the wide range in incidence and prevalence estimates of PSD was reviewed against the background of some major differences in design and methods between studies. Furthermore, evidence for stroke-specific risk factors of PSD is discussed in an attempt to gain insight into the question if PSD is a specific neuropsychiatric disorder that is pathogenically distinct from 'functional' depression.

What is the validity and reliability of commonly used instruments for the diagnosis of depression in a population of stroke patients?

Although a diagnosis of depression should ideally be made on the basis of a standardised diagnostic interview, earlier studies on PSD and community based studies involving large numbers of patients often used self-rating scales for depression to dichotomise patients into diagnostic groups. In. our study we used such self-rating scales as a screening tool for post- stroke depression in order to detect patients at increased risk of PSD (chapter 3). In the case of a positive screening outcome, the patient was further examined using the depression section of the Structured Clinical Interview for DSM-IV (SCID-d).

In this chapter, the accuracy of this procedure, i.e. the predictive validity of these rating scales are analysed using data collected 1 month post-stroke. A diagnosis of PSD (major or minor depression) according to the SCID-d was considered as gold standard.

What is the cumulative one-year incidence of depression after stroke compared to that following myocardial infarction?

Parallel to our cohort of 190 first-ever stroke patients, a cohort of 206 first-ever myocardial infarct patients was studied by our research group using the same methods and prospective design (chapter 4). We considered this MI cohort an appropriate control group, since MI, similar to stroke, is an acute, life-threatening and potentially disabling disease with a vascular aetiology.

The comparison of the incidence rates between both patient groups was meant to challenge the assumption that the reported high incidence of PSD reflects its specific, stroke-related pathogenesis. We argued that this presumption is only 
tenable if the incidence of PSD is indeed higher than that of post-MI depression while controlling for general (aspecific) risk factors such as age, sex, and level of handicap. Combining data from both cohorts, we tested this hypothesis using Cox regression survival analysis. Furthermore, the observed incidence rates of both major and minor depression are described as well as the distribution in time-ofonset of depression.

\section{What is the influence of premorbid personality on the incidence of PSD?}

Five different dimensions of personality are studied as vulnerability factors for PSD: neuroticism, extraversion, openness (to new experiences), conscientiousness, and agreeableness (chapter 5). In depressive disorder, personality characteristics have been intensively studied. Most convincing evidence has been found for neuroticism as a risk factor for depression, while introversion (as opposed to extraversion) has also repeatedly been associated with vulnerability to depression. In line with our hypothesized biopsychosocial model of PSD, we hypothesized that neuroticism increases one's susceptibility to depression after stroke. In addition, recent literature suggests that a personality profile which combines a high level of neuroticism with a low level of extraversion (referred to as type-D personality) predicts a worse outcome in cardiovascular disease patients. Therefore, we further hypothesized an interaction effect between neuroticism and introversion (i.e. low extraversion) on the development of PSD. Both these hypotheses were tested in our cohort of 190 stroke patients, in which personality dimensions were measured after one month using the NEO-FFI, a 60 -item self-rating questionnaire based on the Five Factor Model of personality.

\section{What is the contribution of both focal and generalized vascular brain damage?}

In chapter 6 , both the lesion location hypothesis and the vascular depression hypotheses are evaluated. We attempted to test these two hypotheses in the same cohort, and addressed the question of which of them would be the most likely. As characteristics of focal cerebral damage three variables were selected: side of the lesion (left vs. right hemisphere), involvement of the anterior region of the brain, and territorial vs. lacunar infarcts. As characteristics of generalised cerebrovascular damage, we looked at presence or absence of leukoaraiosis and/or silent cerebral infarcts. Data were collected from CT and MR scans made in the (sub)acute phase of stroke.

In chapter 7 , a re-analysis of the data is presented in which the effects of stroke specific risk-factors for PSD are studied for their additional contribution to the pathogenesis of PSD if they are added to a basic model of non-stroke specific risk 
factors consisting of sex, age, level of handicap, personal history of depression, family history of psychiatric disorders, and somatic comorbidity.

\section{Is PSD adequately recognized and treated in general practice?}

One recent Dutch study reported good correlation between depression scores and general practitioners' judgement about depression in stroke patients. This finding opposed several different reports about poor recognition of depression in somatically compromised patients. Therefore, we interviewed GPs from a subset of 58 stroke patients to see how often PSD was diagnosed by the GPs compared to the outcome from the SCID interviews of our follow-up study. Furthermore, it was attempted to determine which factors were related to misdiagnosis. Moreover, inventory was made of antidepressant treatment either by pharmacotherapy or follow-up consultation (chapter 8).

In chapter 9, the findings presented in this thesis are discussed in a broader perspective. The strengths and weaknesses of the used study design are evaluated and it is considered how these affect the conclusions drawn from the study. It is attempted to integrate the main results of our study with what is currently known about PSD and to explore their implications for both future research and clinical practice. 


\section{CHAPTER 2}

Research into the specificity of depression after stroke: a review of an unresolved issue

This chapter was published as:

Aben I, Verhey F, Honig A, Lodder J, Lousberg R, and Maes M. Research into the specificity of depression after stroke: a review on an unresolved issue. Progress in Neuropsychopharmacology é Biological Psychiatry (2001), 25:671-689 


\section{ABSTRACT}

1. Two decades of research have failed to generate consistent insight into the specificity of post-stroke depression (PSD). This is, at least in part, caused by methodological difficulties.

2. Differences in symptom profiles between PSD and 'normal' depression have been described. However, no specific or unequivocal clinical picture has been established so far.

3. Prevalence rates of PSD varied largely between studies. In community-based studies using standardised diagnostic instruments for depression, relatively low prevalence rates have been reported compared to inpatient or rehabilitation studies. PSD occurs most frequently in the first few months after stroke, while a new incidence peak may occur 2-3 years after stroke.

4. Two systematic reviews on the relationship between lesion location and depression did not support the claim that left hemisphere lesions are a risk factor for PSD. A new concept of vascular depression has been proposed, which relates to depression in the elderly with acute or chronic damage to the cerebral vascular system.

5. Future efforts should aim at increasing the uniformity of study designs, assessment tools should be further improved for use in cognitively impaired patients and appropriate control groups should be defined to study the characteristic features of PSD. 


\section{INTRODUCTION}

Cognitive, emotional and behavioural consequences of stroke have long been recognised. Some of these are specifically provoked by the brain damage itself, such as aphasia or anosognosia. However, in the case of PSD, there has been debate as to whether biological mechanisms are responsible for its development or whether it is mainly a psychological sequel of stroke.

It is thought that stroke may damage cerebral regions or pathways involved in the regulation of emotions. This point of view has been most strongly advanced by Robinson and his co-workers, who were the first to describe a relationship between PSD and the site of the lesion. ${ }^{29}$ On the other hand, it has been argued that depression is also common in the course of other non-cerebral diseases and that, in the elderly, depression is mainly related to the severity of disability and handicap. ${ }^{30}$ Insight into this issue is clinically relevant, as it may shed light on the pathogenesis of PSD, possibly leading to new therapeutic strategies and thus improving the poststroke course and prognosis. Depressed stroke patients profit less from rehabilitation programs 31,32 and have an increased risk of dying within a few months or years after their stroke. ${ }^{27,28}$

During the last two decades, many studies have been published concerning the frequency, phenomenology, course and risk factors of PSD. However, a number of critical reviews have illustrated inconsistent findings on almost all of these aspects. ${ }^{22}, 33-38$ Several sources of bias may explain these inconsistent results. A lack of uniformity in stroke populations, diagnostic criteria, duration of follow up and instruments used to measure depression, hamper the comparability of research findings.

The purpose of this review is to highlight the methodological difficulties complicating research on PSD. The specificity of the relation between stroke and depression is discussed including frequency, phenomenology and pathophysiology of PSD. Finally, recommendations for future research are proposed.

\section{METHODOLOGICAL DIFFICULTIES}

\section{Defining The STRoke Population}

In most studies on PSD, stroke has been regarded as a rather uniform concept, although it constitutes a heterogeneous group of disorders. Aetiology, pathogenesis, symptomatology, course and outcome vary widely among patients. ${ }^{39}$, ${ }^{40}$ Furthermore, the medical care offered to stroke patients largely depends on the nature and severity of the consequences of stroke. Also, the organisation of the care facilities differ between and even within countries. ${ }^{41,}{ }^{42}$ Therefore, selection 
bias occurs if patients are recruited from a specific subpopulation receiving a particular kind of care facility.

Accurate data on the epidemiology of PSD can only be obtained from unselected samples of accurately diagnosed stroke patients. In PSD literature there are no commonly accepted or recognised standards for diagnosing stroke consistently. ${ }^{34,43}$ In addition to misdiagnoses, patient selection criteria have varied widely between studies. ${ }^{34}$ Some authors used restrictive selection criteria to include a homogenous patient group, while others preferred broader criteria. Characteristics, which are sometimes used for patient selection, are the type of stroke (haemorrhage vs. infarction), the site and size of the stroke, a prior history of stroke or other intracerebral diseases, a prior history of depression or substance abuse and cognitive functioning.

As a result of these factors that contribute to the heterogeneity of stroke samples, the generalisation of research findings in stroke patients is to be questioned.

\section{DEFINING PSD}

There is no consensus concerning the diagnostic criteria of PSD. ${ }^{33,44}$ The validity of PSD, if defined by traditional criteria from the DSM-III ${ }^{+5}$ or later versions, can be questioned for several reasons.

The diagnostic classification system of the DSM defines psychiatric syndromes irrespective of their aetiology. However, in case of mental disorders concurring with a somatic disease, this non-etiological basis is not consistently followed. It is unclear why the presence of a presupposed etiological factor should exclude the diagnosis of major depressive disorder. In the case of PSD, the DSM allows for a diagnosis of 'organic mood syndrome, depressed type' (DSM-III-R) ${ }^{46}$ or 'mood disorder due to stroke, with depressive features' or 'with a major depressive-like episode' (DSM-IV). ${ }^{47}$ In research, PSD was mostly defined as either major or minor depression according to the Research Diagnostic Criteria. ${ }^{48}$ This was done because the concept of organic mood syndrome lacked the syndromal specificity of affective disorders and prevented discrimination between different types of depression. ${ }^{49}$

The symptom criteria for major and minor depression, however, have been determined for relatively young depressed patients who are otherwise healthy. The inclusion criteria are strict and have a high threshold in order to optimise diagnostic agreement and reproducibility, while consequently ignoring patients with less severe symptoms. Moreover, applying the syndrome definitions of major and minor depression presupposes that PSD is characterised by the same symptoms.

Although specific symptoms of the DSM criteria may be especially frequent after stroke (e.g. somatic or cognitive) and other relatively rare (e.g. feelings of guilt or thoughts of suicide), it would be a more valuable approach to make a broader 
inventory of psychiatric complaints. This would allow PSD to elicit particular symptoms of its own, which are not part of the standard diagnostic criteria. To study characteristic symptoms of PSD properly, one need not only to compare its profile with that of non-depressed stroke patients but also with that of depressed patients without suffering stroke but with another, chronically invalidating, somatic disease.

A problem is that many symptom criteria of the DSM involve unspecific somatic complaints, which also commonly occur in non-depressed stroke patients. ${ }^{50-52}$ Two approaches have been suggested to get round this problem. Endicott (1984) proposed to exclude the somatic symptoms from the criteria for depression in the medically ill. ${ }^{53}$ Spitzer et al. (1992) and Robins et al. (1981) suggested counting somatic symptoms towards the diagnosis of depression if judged "not to be due primarily to the medical illness". $.5,55$

Similar to somatic symptoms, cognitive complaints may be directly related to stroke. ${ }^{56}$ Clinically, it may be difficult to differentiate cognitive from depressive oçcurrences of stroke. For instance, anhedonia may be regarded as part of a depressive syndrome but also as a lack of initiative as part of a subcortical cognitive syndrome. Slowness of speech and/or thinking may be a sign of depressive psychomotor retardation as well as a direct result of a deficit in cognitive processing. It is questionable whether these phenomena are intrinsically distinct from each other or if they are in fact identical features with different labels..$^{57}$

In addition, depression may be masked by symptoms such as aphasia, anosognosia, denial or aprosody. ${ }^{58,}{ }^{59}$ Stroke symptoms may also mimic depression. ${ }^{33}$, 35 For instance, poor facial expression, motor or speech retardation, loss of initiative, emotionalism and catastrophic or indifferent reactions may lead to false-positive diagnoses.

In absence of an unequivocal concept of PSD, its diagnosis on the basis of a welldefined set of symptoms is still problematic. We believe that more efforts should be undertaken to describe the full range of both depressive and other (neuro)psychiatric symptoms in the course of stroke and to find out which symptom clusters have diagnostic validity in PSD. To be able to differentiate between depression and other sequels of stroke, standardised instruments should be developed to measure these different disorders.

\section{INSTRUMENTS TO ASSESS PSD}

As no accurate biological markers of depression exist, the assessment of depression depends on both history taking and observational : judgement.' Frequently, standardised instruments, such as the PSE, are used which allow for DSM diagnosis of depression. However, several authors applied DSM criteria of depression without using a standardised interview. ${ }^{60-60}$ Many studies measured 
severity of depressive symptoms (rather than depressive disorders) using self-rating scales for depression, such as the Zung-SDS, ${ }^{67}$ the CES-D, ${ }^{68}$ or the BDI. ${ }^{9}, 69-78$ Attempts to diagnose depression on the bases of such scales have been proven to be inaccurate. $31,79-85$ The flaws of the above assessment procedures have led to the development of some specialised instruments. ${ }^{38,58}$

Gordon et al. (1991) introduced the Structured Assessment of Depression in Brain Damaged Individuals (SADBD). This instrument was designed to optimise the reliability of self-report assessment. Questions were put in a factual style requiring only yes or no responses and if necessary, visual cue cards with simple words were supplemented. Despite these valuable adaptations to cognitively impaired patients, the SADBD has not become a widely adopted instrument. Its use is limited by the lack of graded response options and it is not suitable in patients with severe comprehension impairments. 58,86

Instead of relying on a patient's self-report, scales have been developed which are based on clinical observations by trained examiners. An example of such a scale is the Stroke Aphasia Depression Questionnaire, but this has neither been sufficiently validated nor has it been incorporated in many study designs since its introduction in $1998 .{ }^{87}$ The PDRS was designed in an attempt to measure a broader range of emotional, affective and vegetative disturbances after stroke. 88 It consists of 10 different sections, each covering a specific aspect of mood-related symptoms, including catastrophic reactions and hyperemotionalism. Since the PDRS relies not only on observations but also on history taking, its strength does not lie in overcoming reliability problems but in accounting for specific symptom profiles.

A complementary method to increase the diagnostic accuracy of PSD can be sought in the judgement of a patient's closest relative. This approach was originally used in demented patients and adopted by stroke researchers. An example of such an instrument is the Neuropsychology Behaviour and Affect Profile, designed to assess personality changes (e.g. indifference, inappropriateness, and depression) after brain damage. ${ }^{89}$ However, the usefulness of this approach has received too little attention to judge its value in the field of PSD.

To summarise, the assessment of PSD is complicated by multiple difficulties that have insufficiently been managed in most research done so far. Several attempts have been made to refine diagnostic methods using different strategies, in order to increase the reliability of diagnosis and to implement specific clinical presentations of PSD. Ongoing efforts into the improvement of such instruments could significantly improve the diagnostic accuracy of PSD.

\section{START AND DURATION OF FOLLOW-UP}

Different studies used different lengths of follow-up. This clearly influences the outcome of frequency measures. It has even been suggested that, depending on the 
onset of depression after stroke, distinction should be made between early and late onset of depression. This assumption was based on differences in associated factors between these two.

In early onset PSD (within the first month after stroke), functional, physical, and cognitive impairment as well as social functioning were less strongly associated with the severity of depression than in depression that occurred only six months after stroke. ${ }^{90,91}$ However, these findings could not be replicated. ${ }^{92}$

If early onset PSD is distinct from late onset PSD, phenomenology and pathophysiology should be studied separately in both conditions.

\section{PHENOMENOLOGY}

Lipsey et al. (1986) compared post-stroke major depression with 'functional' depression (without a known medical cause) using both the PSE and HAM-D. They found that both patients groups appeared to have almost identical symptom profiles. Only symptoms of slowness were found more frequently in PSD than in 'functional' depression (69\% vs. 30\%), whereas lack of interest and concentration were less frequent (70\% vs. 98\%) according to PSE syndrome scores. ${ }^{9.3}$ On the other hand, based on HAM-D ratings, Andersen et al. (1994) found PSD patients to be significantly more depressed with loss of work and interests, as well as initial insomnia, retardation, gastrointestinal symptoms and general somatic symptoms, than depressed patients without stroke. However, this same pattern emerged when comparing non-depressed stroke patients with non-depressed controls. ${ }^{94}$

Fedoroff et al. (1991a) compared PSD with depression in patients with myocardial infarction and patients with spinal cord lesions. They reported generalised anxiety and ideas of reference to be more common in PSD. 95

Few authors tried to study the diagnostic specificity of somatic symptoms by comparing depressed and non-depressed stroke patients. First, Fedoroff et al. (1991b) compared the frequency of psychological and "autonomic" symptoms (e.g. weight loss, insomnia, loss of energy) of the HAM-D ${ }^{96}$ between 205 patients with and without a depressed mood. They found that both symptom clusters were significantly higher in the depressed group $(n=85)$. If patients had to have one or even two extra autonomic symptom(s) than the minimum required by the DSMIII, ${ }^{45}$ the rate of major depression only decreased with one or two percent respectively. This indicates that PSD is not frequently overdiagnosed due to aspecific autonomic symptoms. ${ }^{50}$ In a later study these findings were shown to be consistent over a two year follow-up period. ${ }^{52}$

Stein et al. (1996) stressed that group differences in the frequency of symptoms do not automatically imply that these symptoms are of diagnostic use. To study if somatic symptoms discriminate PSD patients from non-depressed stroke patients, 
TABLE 1A. Prevalence rates of PSD grouped by setting, diagnostic instruments, and time of assessment: $0-1$ month after stroke

\begin{tabular}{|c|c|c|c|c|c|}
\hline Setting & Publication & $\mathbf{N}$ & Instrument & Time after stroke & Prevalence \\
\hline \multirow[t]{3}{*}{$\begin{array}{l}\text { Com- } \\
\text { muni- } \\
\text { ty }\end{array}$} & $\begin{array}{l}\text { House et al., } 1990100 \\
\text { House et al., } 1991^{125}\end{array}$ & 89 & DSM-III, PSE & 1 month & $\begin{array}{l}11 \% \text { major } \\
1 \% \text { dysthymic } \\
8 \% \text { adjustment }\end{array}$ \\
\hline & & 76 & $\mathrm{BDI}$ & 1 month & $\begin{array}{l}32 \% \text { BDI } \geq 10 \\
20 \% \text { BDI } \geq 13 \\
8 \% \text { BDI } \geq 17\end{array}$ \\
\hline & Wade et al., 198772 & 379 & $\begin{array}{l}\text { Wakefield-SADI } \\
\text { (score 15-18) } \\
\text { Wakefield-SADI } \\
\text { (score 19-36) }\end{array}$ & 3 weeks & $\begin{array}{l}11 \% \text { "probably } \\
\text { depressed" } \\
22 \% \text { "depressed" }\end{array}$ \\
\hline \multirow[t]{8}{*}{$\begin{array}{l}\text { Hos- } \\
\text { pital }\end{array}$} & $\begin{array}{l}\text { Downhill and Robin- } \\
\text { son, } 1994^{115}\end{array}$ & 309 & DSM-III, PSE & $11.2(+/-9.3)$ days & $\begin{array}{l}20 \% \text { major } \\
18 \% \text { minor }\end{array}$ \\
\hline & Fedoroff et al., $1991^{50}$ & 205 & DSM-III, PSE & $<2$ weeks & $\begin{array}{l}22 \% \text { major } \\
? ? \% \text { minor }\end{array}$ \\
\hline & Robinson et al., $1983^{104}$ & 103 & DSM-III, PSE & $11(+/-10)$ days & $\begin{array}{l}27 \% \text { major } \\
20 \% \text { minor }\end{array}$ \\
\hline & Herrmann et al., $1995^{64}$ & 47 & DSM-III-R, n.s.i. & $<2$ months & $\begin{array}{l}19 \% \text { major } \\
17 \% \text { minor }\end{array}$ \\
\hline & Astrom et al., $1993^{61}$ & 76 & DSM-III, n.s.i. & during admission & $\begin{array}{l}25 \% \text { major } \\
? ? \% \text { minor }\end{array}$ \\
\hline & Morris et al., $1996^{73}$ & 193 & $\begin{array}{l}\text { CES-D } \\
\text { (cut-off } \geq 16 \text { ) }\end{array}$ & $7-10$ days & $24 \%$ "depressed" \\
\hline & $\begin{array}{l}\text { Ramasubbu et al., } \\
1998^{78}\end{array}$ & 626 & $\begin{array}{l}\text { CES-D } \\
\text { (cut-off } \geq 16 \text { ) }\end{array}$ & $7-10$ days & $26 \%$ "depressed" \\
\hline & $\begin{array}{l}\text { Andersen et al., } \\
1994^{94}\end{array}$ & 209 & 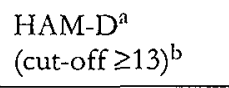 & 1 month & $21 \%$ "depressed" \\
\hline \multirow{2}{*}{$\begin{array}{l}\text { Hos- } \\
\text { pital }+ \\
\text { Rehab- } \\
\text { ilita- } \\
\text { tion }\end{array}$} & Starkstein et al., $1989^{32}$ & 93 & DSM-III, PSE & ca. 15 days $(<60)$ & $\begin{array}{l}18 \% \text { major } \\
12 \% \text { minor }\end{array}$ \\
\hline & Gainotti et al., 199966 & 58 & DSM-III-R, n.s.i. & $<2$ months & $\begin{array}{l}27 \% \text { major } \\
? ? \% \text { minor }\end{array}$ \\
\hline
\end{tabular}

a HAM-D only administered after screening on basis of BDI and nurse teport

b Diagnosis of depression only if complaints persistent during at least two weeks n.s.i. = non(-validated) structured interview 
sensitivity and specificity are better indices. The authors found that in 189 stroke patients the somatic items of both the BDI and HAM-D were less specific when diagnosing PSD than were the non-somatic items. The overall specificity of the somatic items of the BDI was only $46.7 \%$, compared to $80 \%$ for the non-somatic items. For the HAM-D these were $69 \%$ and $86.7 \%$ respectively. Somatic symptoms were, however, similarly sensitive to detect PSD than were non-somatic symptoms. ${ }^{51}$

Finally, attention has been drawn to the distinction between major and minor PSD. Different aetiologies, phenomenologies and course for these two mood states have been suggested. For instance, Starkstein and Robinson (1989) found only a strong association between major depression and lesions of the left frontal cortex or basal ganglia. Major depression was shown to remit earlier than minor depression and dementia of depression (pseudo dementia) was reported to result from major but not from minor PSD. ${ }^{32}$ Minor depression, on the other hand was found to be associated with younger age and more caudal hemisphere lesions. ${ }^{+4}$.

\section{PREVALENCE}

Many prevalence estimates for PSD have been reported during the last two decades. These range between $10 \%$ and $64 \%$. Despite the large spread of these estimates, they do illustrate that stroke increases the risk of depression. The prevalence of major depression in the community lies between $2-4 \%$ and is only raised to $6-14 \%$ on inpatient medical wards. ${ }^{97}$ Recent evidence from the Epidemiologic Catchment Area Study showed even decreased rates of current depressive disorder among the elderly, the age group in which stroke most frequently occurs. ${ }^{98,99}$

In order to cautiously interpret the prevalence rates found in PSD research, the authors subjected a number of studies to a more detailed analysis. We compared prevalence estimates by taking three potential sources of bias into account: study setting/population, diagnostic depression instrument and time-point of assessment after stroke (Table 1a-d).

\section{Community STUdies}

As can be seen from table 1 , only a few publications studied community based stroke samples using validated instruments, which allow for a diagnosis of depressive disorder. Both the Oxford Community Study 100,101 and the Perth Community Study ${ }^{102,} 103$ found relatively low prevalence rates compared to studies based on hospital and/or rehabilitation samples. If studies, which use self-report 
TABLE 1B. Prevalence rates of PSD grouped by setting, diagnostic instruments, and time of assessment: $2-4$ months after stroke

\begin{tabular}{|c|c|c|c|c|c|}
\hline Setting & Publication & $\mathbf{N}$ & Instrument & Time after stroke & Prevalence \\
\hline \multirow{3}{*}{$\begin{array}{l}\text { Com- } \\
\text { muni- } \\
\text { ty }\end{array}$} & Burvill et al., $1997^{103}$ & 191 & DSM-III, PAS & 4 months & $\begin{array}{l}17 \% \text { major } \\
11 \% \text { minor }\end{array}$ \\
\hline & Burvill et al., $1995^{102}$ & 294 & DSM-III, PAS & 4 months & $\begin{array}{l}15 \% \text { major } \\
8 \% \text { minor }\end{array}$ \\
\hline & $\begin{array}{l}\text { Kotila et al., } \\
1998 / 99^{77}, 126\end{array}$ & 321 & $\mathrm{BDI}(\geq 10)$ & 3 months & $47 \%$ "at risk" \\
\hline \multirow[t]{7}{*}{$\begin{array}{l}\text { Hos- } \\
\text { pital }\end{array}$} & $\begin{array}{l}\text { Pohjasvaara et al., } \\
1998^{107}\end{array}$ & 277 & $\begin{array}{l}\text { DSM-III-R, } \\
\text { SCAN/PSE }\end{array}$ & 3.4 months & $\begin{array}{l}26 \% \text { major } \\
14 \% \text { minor }\end{array}$ \\
\hline & Morris et al., $1990^{108}$ & 99 & DSM-III, CIDI & $\begin{array}{l}2 \text { months } \\
\text { (max } 21 \text { weeks) }\end{array}$ & $\begin{array}{l}14 \% \text { major } \\
18 \% \text { minor }\end{array}$ \\
\hline & Kauhanen et al., 199965 & 101 & DSM-III-R, n.s.i. & 3 months & $\begin{array}{l}9 \% \text { major } \\
44 \% \text { minor }\end{array}$ \\
\hline & Astrom et al., $1993^{61}$ & 73 & DSM-III, n.s.i. & 3 months & $\begin{array}{l}31 \% \text { major } \\
? ? \% \text { minor }\end{array}$ \\
\hline & Spencer et al., $1995^{\prime \prime \prime}$ & 162 & CES-D & $3-10$ weeks & $33 \%$ "at risk" \\
\hline & Andersen et al., $1994^{94}$ & 204 & 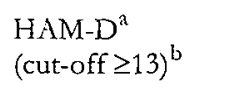 & 3 months & $15 \%$ "depressed" \\
\hline & Herrmann et al., $1998^{76}$ & 150 & $\begin{array}{l}\text { MADRS } \\
\text { (cut-off } \geq 7 \text { ) } \\
\text { Zung-SDS } \\
\text { (cut-off } \geq 50 \text { ) }\end{array}$ & 3 months & $\begin{array}{l}27 \% \text { "marked } \\
\text { symptoms" } \\
22 \% \text { "marked } \\
\text { symptoms" }\end{array}$ \\
\hline $\begin{array}{l}\text { Hosp./ } \\
\text { Rehab. }\end{array}$ & Gainotti et al., 199966 & 52 & DSM-III-R, n.s.i. & $2-4$ months & $\begin{array}{l}27 \% \text { major } \\
? ? \% \text { minor }\end{array}$ \\
\hline \multirow[t]{4}{*}{$\begin{array}{l}\text { Reha- } \\
\text { bilita- } \\
\text { tion }\end{array}$} & Eastwood et al., 1989 & 87 & RDC, SADS & $\begin{array}{l}82.1(+/-58.5) \text { days } \\
\text { and ca. } 4 \text { months } \\
\text { later/on discharge }\end{array}$ & $\begin{array}{l}10 \% \text { major } \\
40 \% \text { minor }\end{array}$ \\
\hline & Gordon et al., $1991^{58}$ & 116 & $\begin{array}{l}\text { DSM-III, } \\
\text { SADBD }\end{array}$ & $\begin{array}{l}8.4 \text { weeks } \\
\text { (> } 4 \text { weeks) }\end{array}$ & $\begin{array}{l}32 \% \text { major } \\
32 \% \text { minor }\end{array}$ \\
\hline & Morris et al, $1992^{11 \prime \prime}$ & 88 & DSM-III, CIDI & 2 months & $\begin{array}{l}18 \% \text { major } \\
20 \% \text { minor }\end{array}$ \\
\hline & Sinyor et al., $1986^{70}$ & 64 & Zung-SDS & $57(+/-?)$ days & $\begin{array}{l}25 \% \text { "mild" } \\
22 \% \text { "moderate- } \\
\text { severe" }\end{array}$ \\
\hline
\end{tabular}

a HAM-D only administered after screening on basis of BDI and nurse report $\mathrm{b}$ diagnosis of depression only if complaints persistent during at least two weeks n.s.i. $=$ non(-validated) structured interview 
scales, are also taken into account, the range of prevalence rates clearly increases, suggesting that these instruments are less diagnostically accurate. ${ }^{72,77}$

After the acute phase, the prevalence of PSD decreased in the Oxford Community Study. After six months $15 \%$ were found to have depression and after one year this was further reduced to $10 \%, 100,101$ Community studies with diagnoses based on self-report data, higher rates of about $20-50 \%$ were found after 6 months to one year. ${ }^{72,} 77$

\section{HOSPITAL/REHABILITATION STUDIES}

In hospitalised patients, the prevalence of major depression ranged from 19-27\% in the acute post-stroke phase (based on DSM-III diagnoses). $50,61,64,104,105$ Similar prevalence rates were reported in a number of self report studies ${ }^{73,78,94}$ and in two studies in which both inpatients and rehabilitation patients were recruited. ${ }^{66,106}$

TABLE 1C. Prevalence rates of PSD grouped by setting, diagnostic instruments, and time of assessment: 6 months after stroke

\begin{tabular}{|c|c|c|c|c|c|}
\hline Setting & Publication & $\mathbf{N}$ & Instrument & Time after stroke & Prevalence \\
\hline \multirow[t]{8}{*}{ Community } & \multirow{6}{*}{$\begin{array}{l}\text { House et al., } \\
1990 / 91^{1(1), 125}\end{array}$} & \multirow[t]{3}{*}{119} & \multirow[t]{3}{*}{ DSM-III, PSE } & \multirow[t]{6}{*}{6 months } & $9 \%$ major \\
\hline & & & & & $3 \%$ dysthymic \\
\hline & & & & & $3 \%$ adjustment \\
\hline & & \multirow[t]{3}{*}{107} & \multirow[t]{3}{*}{ BDI } & & $32 \%$ BDI $\geq 10$ \\
\hline & & & & & $15 \% \mathrm{BDI} \geq 13$ \\
\hline & & & & & $6 \% \mathrm{BDI} \geq 17$ \\
\hline & \multirow[t]{2}{*}{$\begin{array}{l}\text { Wade et al., } \\
198772\end{array}$} & \multirow[t]{2}{*}{377} & $\begin{array}{l}\text { Wakefield-SADI } \\
\text { (score 15-18) }\end{array}$ & \multirow[t]{2}{*}{6 months } & $\begin{array}{l}12 \% \text { "probably } \\
\text { depressed" }\end{array}$ \\
\hline & & & $\begin{array}{l}\text { Wakefield-SADI } \\
\text { (score 19-36) }\end{array}$ & & $20 \%$ "depressed" \\
\hline \multirow[t]{5}{*}{ Hospital } & \multirow{2}{*}{$\begin{array}{l}\text { Robinson et al., } \\
1984 c^{91}\end{array}$} & \multirow[t]{2}{*}{61} & \multirow[t]{2}{*}{ DSM-III, PSE } & \multirow[t]{2}{*}{6 months } & $34 \%$ major \\
\hline & & & & & $26 \%$ minor \\
\hline & $\begin{array}{l}\text { Ebrahim et al., } \\
198771\end{array}$ & 149 & $\begin{array}{l}\text { GHQ } \\
\text { (cut-off } \geq 12 \text { ) }\end{array}$ & 6 months & $\begin{array}{l}23 \% \text { "important } \\
\text { affective illness" }\end{array}$ \\
\hline & $\begin{array}{l}\text { Spencer et al., } \\
1995111\end{array}$ & 140 & CES-D & 6 months & $25 \%$ "at risk" \\
\hline & $\begin{array}{l}\text { Andersen } \text { et al., } \\
1994^{94}\end{array}$ & 199 & $\begin{array}{l}\text { HAM-D }^{a} \\
\text { (cut-off } \geq 13)^{b}\end{array}$ & 6 months & $9 \%$ depressed \\
\hline
\end{tabular}

a HAM-D only administered after screening on basis of BDI and nurse report $\mathrm{b}$ diagnosis of depression only if complaints persistent during at least two weeks n.s.i. $=$ non(-validated) structured interview 
TABLE 1D. Prevalence rates of PSD grouped by setting, diagnostic instruments, and time of assessment: 1-3 year(s) after stroke

\begin{tabular}{|c|c|c|c|c|c|}
\hline Setting & Publication & $\mathbf{N}$ & Instrument & Time after stroke & Prevalence \\
\hline \multirow[t]{9}{*}{ Community } & \multirow{6}{*}{$\begin{array}{l}\text { House et al., } \\
\text { 1990/9110, } 125\end{array}$} & \multirow[t]{3}{*}{112} & \multirow[t]{3}{*}{ DSM-III, PSE } & \multirow[t]{6}{*}{1 year } & $5 \%$ major \\
\hline & & & & & $4 \%$ dysthymic \\
\hline & & & & & $1 \%$ adjustment \\
\hline & & \multirow[t]{3}{*}{88} & \multirow[t]{3}{*}{ BDI } & & $20 \%$ BDI $\geq 10$ \\
\hline & & & & & $10 \% \mathrm{BDI} \geq 13$ \\
\hline & & & & & $5 \%$ BDI $\geq 17$ \\
\hline & \multirow[t]{2}{*}{$\begin{array}{l}\text { Wade et al., } \\
198772\end{array}$} & \multirow[t]{2}{*}{348} & $\begin{array}{l}\text { Wakefield-SADI } \\
\text { (score 15-18) }\end{array}$ & \multirow[t]{2}{*}{1 year } & $\begin{array}{l}13 \% \text { "probably } \\
\text { depressed" }\end{array}$ \\
\hline & & & $\begin{array}{l}\text { Wakefield-SADI } \\
\text { (score 19-36) }\end{array}$ & & $18 \%$ "depressed" \\
\hline & $\begin{array}{l}\text { Kotila et al., } \\
1998^{77}\end{array}$ & 311 & $\mathrm{BDI}(\geq 10)$ & 1 year & $47 \%$ "at risk" \\
\hline \multirow[t]{14}{*}{ Hospital } & $\begin{array}{l}\text { Morris et al., } \\
1990^{108}\end{array}$ & 56 & DSM-III, CIDI & $\begin{array}{l}17 \text { months } \\
\text { (M } 67.3 \text { weeks; } \\
\max 2 \text { years.) }\end{array}$ & $\begin{array}{l}7 \% \text { major } \\
5 \% \text { minor }\end{array}$ \\
\hline & \multirow{2}{*}{$\begin{array}{l}\text { Robinson et al., } \\
1987112\end{array}$} & \multirow[t]{2}{*}{37} & \multirow[t]{2}{*}{ DSM-III, PSE } & \multirow[t]{2}{*}{1 year } & $14 \%$ major \\
\hline & & & & & $19 \%$ dysthymic \\
\hline & \multirow{2}{*}{$\begin{array}{l}\text { Kauhanen et al., } \\
1999^{65}\end{array}$} & \multirow[t]{2}{*}{92} & \multirow[t]{2}{*}{ DSM-III-R, n.s.i. } & \multirow[t]{2}{*}{1 year } & $16 \%$ major \\
\hline & & & & & $26 \%$ minor \\
\hline & \multirow{2}{*}{$\begin{array}{l}\text { Astrom et al., } \\
1993^{61}\end{array}$} & \multirow[t]{2}{*}{68} & \multirow[t]{2}{*}{ DSM-III, n.s.i. } & \multirow[t]{2}{*}{1 year } & $16 \%$ major \\
\hline & & & & & ??\% minor \\
\hline & \multirow[t]{2}{*}{$\begin{array}{l}\text { Herrmann et al., } \\
1998^{76}\end{array}$} & \multirow[t]{2}{*}{136} & $\begin{array}{l}\text { MADRS } \\
\text { (cut-off } \geq 7 \text { ) }\end{array}$ & \multirow[t]{2}{*}{1 year } & $\begin{array}{l}22 \% \text { "marked } \\
\text { symptoms" }\end{array}$ \\
\hline & & & $\begin{array}{l}\text { Zung-SDS } \\
\text { (cut-off } \geq 50 \text { ) }\end{array}$ & & $\begin{array}{l}21 \% \text { "marked } \\
\text { symptoms" }\end{array}$ \\
\hline & $\begin{array}{l}\text { Andersen et al., } \\
1994^{94}\end{array}$ & 191 & 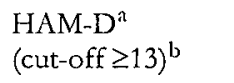 & 1 year (cross-sect.) & $5 \%$ depressed \\
\hline & \multirow{2}{*}{$\begin{array}{l}\text { Robinson et al., } \\
1987112\end{array}$} & \multirow[t]{2}{*}{48} & \multirow[t]{2}{*}{ DSM-III, PSE } & \multirow[t]{2}{*}{2 years } & $21 \%$ major \\
\hline & & & & & $21 \%$ minor \\
\hline & \multirow{2}{*}{$\begin{array}{l}\text { Astrom et al., } \\
1993^{61}\end{array}$} & 57 & \multirow[t]{2}{*}{ DSM-IIY, n.s.i. } & 2 years & $19 \%$ major \\
\hline & & 49 & & 3 years & $29 \%$ major \\
\hline
\end{tabular}

a HAM-D only administered after screening on basis of BDI and nurse report $b$ diagnosis of depression only if complaints persistent during at least two weeks n.s.i. $=$ non(-validated) structured interview 
Post-stroke minor depression at this early time-point ranged from $12-20 \%$, whereas the overall prevalence (major and minor combined) ranged from 30-47\%. ${ }^{64,104-106}$ After 2-4 months the numbers seem not to be very different (major depression 9$31 \%, 61,65,66,107,108$ minor depression in $14-44 \% ; 61,65,66,107,108$ overall $32-58 \%$ ). In rehabilitation patients, depression is equally or only slightly more frequent (major depression 10-32\%; minor depression $20-40 \%$; overall $38-64 \%$ ). $58,109,110$

At six months post-stroke, Robinson et al. (1984b) reported a high overall prevalence rate of $60 \%$ based on DSM-III criteria. ${ }^{90}$ On the other hand, using rating scales which measure the severity of depressive symptoms, clearly lower rates were found ranging from $9-25 \% .{ }^{71,94,111}$

In the more chronic course of stroke $(\geq 1$ year) the prevalence of depression first seems to decrease, as was seen in the community based studies, but after two to three years it seems to increase again. Three studies which followed their patients one to three years after stroke found major depression in 7-16\% after 1-1.5 years, ${ }^{61}$, $65,108,112$ in $19-21 \%$ after 2 years ${ }^{61,112}$ and in $29 \%$ after 3 years. ${ }^{61}$

No data is available on the cumulative incidence of PSD, which is the most informative measure of the frequency of depression after stroke. Andersen et al. (1994) were the only group who calculated the one-year prevalence of PSD. They reported 23\% major depression and 18\% "less than major depression" based on HAM-D $\leq 13$ and complaints persisting for at least two weeks. ${ }^{94}$

Since the recruitment of patients starts only after the stroke has occurred, no data is available on the pre-stroke mood state of these patients. Therefore, depressive episodes that already exist at the time of the stroke cannot reliably be detected and may blur the true incidence of PSD. Moreover, the influence of excluding patients with communication disabilities and of misdiagnosing depression in patients with aprosody, neglect, et cetera, on true incidence estimates, remains unknown.

\section{PATHOPHYSIOLOGY}

Much research has been carried out to define the pathophysiological correlates of PSD. 36 These studies have been based on two main hypotheses on the relationship between depression and stroke: "lesion location" versus "psychological reaction".

Different reviews, however, showed that the results of all these studies are inconclusive. $22,35-37,113$

When evaluating the specificity of cerebrovascular damage to subsequent depression, factors which determine or modify the psychological reaction to the stroke (e.g. severity of disability and handicap, social functioning, coping strategies, personality traits) should be considered as aspecific, since they are not exclusively restricted to stroke. Vulnerability factors such as age, sex, former life-events, and prior history or family history of psychiatric disorders must also be seen in that 
perspective. These factors should, however, be controlled whilst searching for specific stroke-related phenomena that explain the development of PSD.

\section{LESION LOCATION HYPOTHESIS}

Robinson and his group reported that left hemisphere lesions were more frequently associated with depression than right hemisphere lesions.9 29, 114 This was the case for left anterior lesions ${ }^{29}$ and consistent for both cortical and subcortical lesions. ${ }^{115}$ To explain these findings, it was hypothesised that the relationship between anterior lesion location and depression consists of a distuption of noradrenergic and serotonergic pathways. These neurotransmitter systems originate in the brainstem and run anteriorly (through the lateral hypothalamus and basal ganglia) to the frontal cortex. From here, they spread posteriorly to almost the entire cortex. This distuption of noradrenergic and serotonergic pathways was thought to explain that lesions in the frontal cortex or basal ganglia would be more likely to induce depression than posterior lesions. ${ }^{32,49}$ However, this explanation did not clarify the differences between left and right sided lesions.

Recently, two systematic reviews were published on lateralisation effects in PSD. Both studies offered no support for the hypothesis that the risk of depression depends on the hemispheric location of the lesion. ${ }^{22,23}$ However, these studies did not take the time of onset of PSD into account. As was already stressed (see paragraph 2.4) early-onset PSD may show a different pathogenesis from late-onset PSD. It is conceivable that lesion location plays a role in the development of PSD immediately after stroke, whereas in late-onset PSD failure to adjust to the consequences of stroke may be more important.

\section{VASCular Depression Hypothesis}

Based on different observations, it has recently been hypothesised that depression in the elderly arises as a result of acute or progressive damage to the cerebral vascular system. ${ }^{116}$ PSD may be an example of a vascular disease in which this may occur.

Depression was shown to be highly prevalent in patients with diseases involving the cardiovascular system, such as diabetes, myocardial infarction, coronary artery disease ${ }^{117}$ and, hypertension. ${ }^{118}$ Depression in the elderly is associated with silent cerebral infarctions ${ }^{119,120}$ and white matter hyperintensities on MRI scans. These hyperintensities are indicative of damage to small vessels in these patients. ${ }^{121,} 122$ Furthermore, vascular risk factors have been reported to be more frequent in patients with late-onset depression, ${ }^{123}$ whereas a positive family history of depression was found to be less frequent in depressed patients with silent stroke. ${ }^{124}$ Those vascular diseases, which can exist for years without any clinical manifestations (e.g. hypertension and silent cerebral infarctions), are especially 
interesting. The absence of psychological stress as a reaction to the illness may indicate a more biological origin of depression in these patients.

Thus, the vascular depression hypothesis might be a valid new concept, making it worthwhile to pay attention to the involvement of vascular risk factors in the pathophysiology of PSD.

\section{CONCLUSIONS}

At present the question concerning the specificity of PSD cannot be answered satisfactorily. This has, at least in part, been caused by methodological difficulties. To gain insight into this topic, these methodological issues should be addressed. Moreover, it is crucial to define appropriate control groups of depressed patients who have not suffered a stroke but eventually suffered other chronically invalidating somatic disorders. Consensus on uniform patient selection criteria, diagnostic criteria and diagnostic instruments would improve the comparability of studies.

Specific clinical features of PSD have, as yet, not been agreed upon. The prevalence of PSD was reported to vary widely but it clearly seems to depend on study settings and time after stroke. Recent meta-analyses revealed that there is insufficient support for the lesion location hypothesis. The vascular depression hypothesis may, however, play an important role in the pathogenesis of PSD. 
CHAPTER 3

Validity of the BDI, HADS, SCL-90, and HAM-D as screening instruments for depression in stroke patients

This chapter was published as:

Aben I, Verhey F, Lousberg R, Lodder J, and Honig A. Validity of the BDI, HADS, SCL-90, and HAM-D as screening instruments for depression in stroke patients Psycbosomatics (2002), 43:386-393 


\begin{abstract}
Most instruments used to assess post-stroke depression have never been specifically validated in stroke patients. This study evaluated the screening abilities of three questionnaires and one observer rating scale for depression one month after a first-ever ischemic stroke in 202 consecutive patients. At their respective optimum cut-off values, the sensitivity of the self-rating scales varied between 80 to $90 \%$, while the specificity was about $60 \%$. For the observer rating scale (HAM-D), sensitivity was 78.1 , specificity $74.6 \%$. The instruments performed clearly better in men than women. Despite this difference, we conclude that all scales are acceptable screening instruments for post-stroke depression.
\end{abstract}




\section{INTRODUCTION}

Most scales used to assess post-stroke depression have never been properly validated in stroke patients, ${ }^{3,3}, 36$ which diminishes the force of conclusions based on research data generated with these scales. At least two different aspects of stroke patients potentially disturb the psychometric properties of depression rating scales. First, stroke patients are mainly elderly patients, who frequently have cognitive impairments, and this potentially decreases the reliability of their responses to questions. ${ }^{38}$ Second, as a direct consequence of stroke, patients may suffer from symptoms such as insomnia and loss of appetite, which may lead to an increase in false-positive depression scores. ${ }^{50,51}$

We studied the validity of three self-rating scales and one observer-rating scale as screening or diagnostic instruments for depression in a clinical stroke sample. The scales were the Beck Depression Inventory (BDI), the Hospital Anxiety and Depression Scale (HADS), the 90-items Symptom Checklist (SCL-90), and the Hamilton Depression Rating Scale (HAM-D). Sensitivity, specificity, and predictive values were determined at different cut-off points using DSM-IV ${ }^{47}$ diagnoses of major and minor depressive disorder as gold standard. The internal consistency of the self-rating scales was also studied.

\section{PATIENTS}

Two-hundred-two consecutive patients with a diagnosis of first, hemispheric infarction were included in the study, which was performed by the Department of Psychiatry \& Neuropsychology and the Department of Neurology of the University Hospital of Maastricht, the Netherlands, the only hospital in the vicinity of Maastricht. Patients data were entered into a prospective stroke registry, which has been described in detail elsewhere. ${ }^{127}$ Patients with a comorbid intracerebral disease (e.g. a brain tumour or Parkinson's disease) or a major psychiatric disorder other than affective disorders (e.g. schizophrenia, dementia, or a present psychotic episode) were excluded from this study. Additionally, patients who were unable to communicate reliably (e.g: because of severe aphasia or cognitive dysfunction) were excluded on the basis of combined clinical judgement and Mini Mental State Examination (MMSE) and Frenchay Aphasia Screening Test (FAST) results (see methods section). All participants gave written informed consent. The study was approved by the Medical Ethics Committee of the University Hospital. 


\section{METHODS}

All patients were assessed 1 month after a first-ever stroke. First, MMSE ${ }^{128}$ and FAST $^{129}$ were administered to measure global cognitive functioning. The level of disability and handicap was rated using the Barthel Index ${ }^{130}$ and Rankin score, ${ }^{131}$ respectively. Data concerning level of education, living situation, and marital status were collected on inquiry.

Next, all patients were interviewed using both the depression section of the Structured Clinical Interview for DSM-IV (SCID-I-R) ${ }^{132}$ and the HAM-D. ${ }^{96}$ The SCID is a structured psychiatric diagnostic interview allowing for a diagnosis of depression according to DSM-IV criteria. ${ }^{47}$ Patients were diagnosed as having major depression if they had at least one core symptom (i.e. depressed mood or loss of interest) and at least four other symptoms of depression that had lasted at least 2 weeks. A diagnosis of "minor depression" was made if patients had at least one core symptom and at least two but less than five symptoms in total as defined in the DSM-IV. ${ }^{47}$ The HAM-D is a clinical rating scale, which quantitatively measures the severity of depressive symptoms. All interviews were administered by the same clinician (IA), who was trained to use these instruments.

All eligible patients were asked to fill out the three questionnaires at home after the interview. The delay between the interview and filling out the questionnaires was registered. The $\mathrm{BDI}^{69,133}$ is a 21 -item questionnaire commonly used in research on post-stroke depression. ${ }^{36}$ The HADS ${ }^{129}, 134$ consists of depression and anxiety subscales (further referred to as HADS-D and HADS-A, respectively). Each subscale consists of 7 items. The HADS was specifically developed for use in patients with somatic comorbidity. The SCL-90135, 136 is a self-rating scale which consists of eight domains of psychiatric symptoms, including a 16-item depression subscale (SCL-90-D). In this study, all 90 items were administered. The SCL-90 has been used in stroke patients to measure distress ${ }^{137}$ and as a screening instrument for depression in myocardial infarct patients. ${ }^{138}$

The generally accepted threshold for the BDI is $9 / 10$. In the original publication on the HADS, ${ }^{129}$ a cut-off level of $7 / 8$ was recommended for both subscales separately, to detect both doubtful and definite cases. The HADS total score (both subscales together) has also been used as a general measure of distress. ${ }^{129,134}$ In the case of the SCL-90-D, based on score distributions in a large sample of normal individuals, a threshold of $22 / 23$ for men and $27 / 28$ for women has been established. ${ }^{138}$

To assess the optimum cut-off points, a Receiver Operating Characteristics (ROC) curve was obtained for all scales. ${ }^{139}$ The "area under the curve" (AUC) was calculated as a measure of the overall accuracy of the scale. Additionally, the positive predictive value (PPV) and the negative predictive value (NPV) were calculated for different cut-off scores in the central range of the scales. 
To determine whether the scores of the three self-rating questionnaires were biased by fill out errors, internal consistency, as measured by Cronbach's alpha, was computed for both the whole sample and a subset of patients with MMSE scores of 23 or lower.

\section{RESULTS}

All 202 patients included in the study had a stroke between September 1, 1997 and September 1, 1999. In this period, a total of 462 patients were diagnosed as having a primary hemispheric cerebral infarction. Hundred-ninety-six (42.4\%) of them had to be excluded, mainly because of severe comorbidity (including aphasia and dementia). Sixty-four $(24,1 \%)$ eligible patients refused participation. The major characteristics of the study population are summarized in table 1.

Thirty-two patients (15.8\%) met DSM-IV criteria for major depressive disorder and $19(9.4 \%)$ for minor depressive disorder, corresponding to an overall prevalence of

TABLE 1. Demographic and stroke outcome related characteristics of the patients

\begin{tabular}{ll}
\hline & $\mathrm{n}=\mathbf{2 0 2}$ \\
\hline sex (female) & $45.5 \%$ \\
age, yrs (mean $\pm \mathrm{SD}$ ) & $68.5 \pm 11.6$ \\
level of education & \\
$\quad$ primary school & $41.8 \%$ \\
$\quad$ junior secondary vocational education & $26.3 \%$ \\
$\quad$ senior secondary vocational education & $19.6 \%$ \\
$\quad$ (pre-)university education & $11.8 \%$ \\
marital status $\quad$ & \\
$\quad$ married/living together & $62.4 \%$ \\
$\quad$ widowed & $9.8 \%$ \\
MMSE (median, min-max) & $26.3 \%$ \\
FAST (median, min-max) & $26(16-30)$ \\
Barthel (median, min-max) & $27(9-30)$ \\
Rankin (mean, s.d.) & $20(0-20)$ \\
Major depression & $2.4(1.2)$ \\
Minor depression & $15.8 \%$ \\
Hamilton-D score (mean \pm SD) & $9.4 \%$ \\
\hline
\end{tabular}

MMSE: Mini Mental State Examination (minimum score: 0, maximum score: 30); FAST: Frenchay Aphasia Screening Test (minimum score: 0, maximum scorc: 30); Barthel Index (completely dependent in Activities of Daily Living: 20, no disabilities: 0); Rankin (no handicaps: 0 , bedridden: 5). 
TABLE 2. Screening abilities at optimum cut-off values for major depression alone and for both major and minor depression

\begin{tabular}{lllllllllllll}
\hline & Cut-off & \multicolumn{2}{c}{ Sensitivity } & \multicolumn{2}{c}{ Specificity } & \multicolumn{2}{c}{ PPV } & \multicolumn{2}{c}{ NPV } & \multicolumn{2}{c}{ AUC } \\
\hline & & major & minor & major & minor & major minor & major & minor & major & minor \\
\hline SCL-90-D & $24 / 25$ & 88.5 & 87.8 & 60.7 & 65.9 & 28.0 & 43.9 & 96.8 & 94.7 & 0.81 & 0.85 \\
BDI & $9 / 10$ & 80.0 & 77.1 & 61.4 & 65.4 & 22.2 & 37.5 & 95.7 & 91.4 & 0.78 & 0.79 \\
HADS-D & $7 / 8^{*}$ & 73.1 & 72.5 & 81.6 & 78.9 & 41.3 & 50.9 & 94.5 & 90.5 & 0.82 & 0.83 \\
HADS-A & $4 / 5$ & 91.7 & 88.5 & 56.1 & 71.8 & 25.9 & 63.5 & 97.7 & 91.9 & 0.78 & 0.77 \\
HADS-total & $10 / 11$ & 91.7 & 86.8 & 65.3 & 69.9 & 30.1 & 45.2 & 98.0 & 94.9 & 0.83 & 0.84 \\
HAM-D & $11 / 12$ & 78.1 & 78.4 & 74.6 & 81.3 & 36.8 & 58.8 & 94.7 & 91.7 & 0.83 & 0.86 \\
\hline
\end{tabular}

PPV: Positive Predictive Value; NPV: Negative Predictive Value; AUC: Area Under (ROC-) Curve; SCL-90-D: Symptom Checklist-90, depression subscale; BDI: Beck Depression Inventory; HADS: Hospital Anxiety and Depression Scale; HAM-D: Hamilton Depression Rating Scale; major: only major depression; minor: both major and minor depression; *j $6 / 7$, if screening for both major and minor depression.

$25.2 \%$. Twenty-four (47.1\%) of these were women. Depressed patients had a different HAM-D score from non-depressed patients (major depression 17.6 \pm 7.5 , minor depression 14.9 \pm 4.8 , non-depressed 7.7 $\pm 5.0 ; \mathrm{F}(2)=51.9 ; \mathrm{p}<0.001$ ).

As could be expected, a substantial number of patients had difficulties to fill-out the self-rating scales. Each scale was considered to be completed if at least $75 \%$ of the items were filled out properly. By this definition, 26 patients $(12.9 \%)$ did not complete any of the scales. Patients, who reported to experience difficulties filling out the questionnaires, were encouraged to complete at least the SCL-90. Therefore, the response was slightly higher for this scale $(n=176)$ than for the HADS ( $n=171)$ and the BDI $(n=166)$. Only valid responses were used for the subsequent analysis of the sensitivity, specificity, and predictive values of each scale.

The median delay between the interview and filling out the self-rating scales was 5 days; all but 13 patients returned their questionnaires within 3 weeks after the interview. However, because of logistic problems, 20 patients were interviewed 1 day to three weeks after they had filled out the questionnaires.

The internal consistency of all self-rating scales turned out to be good, with values of Cronbach's alpha of 0.85 for the HADS-D, 0.83 for the BDI, and 0.88 for the SCL-90-D. Analysis of a subset of patients with low scores on the MMSE $(\leq 23$; $\mathrm{n}=43$ ) showed slightly lower values of alpha: HADS-D 0.71, BDI 0.79, and SCL90-D 0.77. Moreover, non-response was significantly higher in this subgroup $(\chi 2(1)=5.9 ; \mathrm{p}=0.02)$.

ROC curves are displayed in figure 1. In screening for major depression, the AUCs of the SCL-90-D, HADS-D, and HAM-D were almost equal, ranging from 0.81 to 
Figure 1. Receiver Operating Characteristics (ROC) curves of the SCL-90, BDI, HADS-D, and HAM-D in screening for major depression. Numbers represent cutoff values

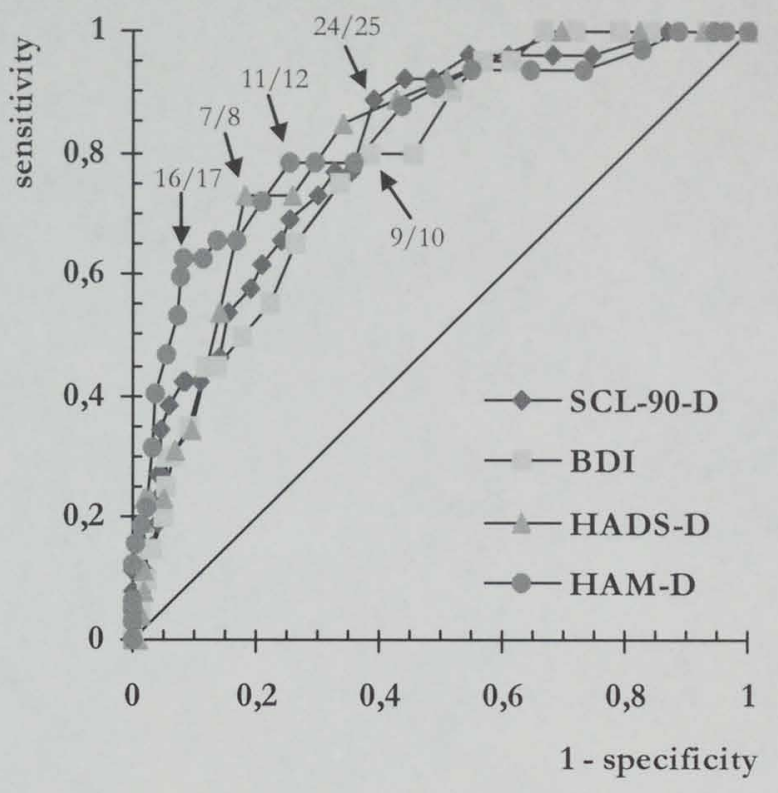

0.83. The AUC of the BDI was only slightly lower (0.78). The anxiety subscale of the HADS and the total HADS were also entered into the ROC analyses (Figure 2). This revealed an AUC of 0.78 for the HADS-A and 0.83 for the total HADS (Table 2).

The optimum cut-off point (with the highest sum of sensitivity and specificity) for the SCL-90-D was 24/25 (sensitivity 88.5 , specificity 60.7), for the BDI it was $9 / 10$ (sensitivity 80.0, specificity 61.4), and for the HADS-D it was 7/8 (sensitivity 73.1, specificity 81.6). Using the total HADS resulted in a sensitivity of no less than 91.7 (specificity 65.3) at its optimum threshold of 10/11 (Table 2).

In the case of the HAM-D, the cut-off value with the highest sum of sensitivity and specificity was 16/17 (sensitivity 62.5, specificity 91.7). However, detailed inspection of the ROC-curve of the HAM-D showed that, especially in the central range, the smoothness of the curve was somewhat less than perfect. The sums of sensitivity and specificity at cut-off values $11 / 12$ and $16 / 17$ were almost equal, whereas the values in between clearly gave poorer results. In comparison to point $16 / 17$, sensitivity improved by $15.6 \%$ to 78.1 at cut-off point $11 / 12$, while the specificity decreased by $17.1 \%$ to 74.6 . 
Figure 2. Receiver Operating Characteristics (ROC) curves of the HADS-D, HADS-A and HADS-total in screening for major depression. Numbers represent cut-off values

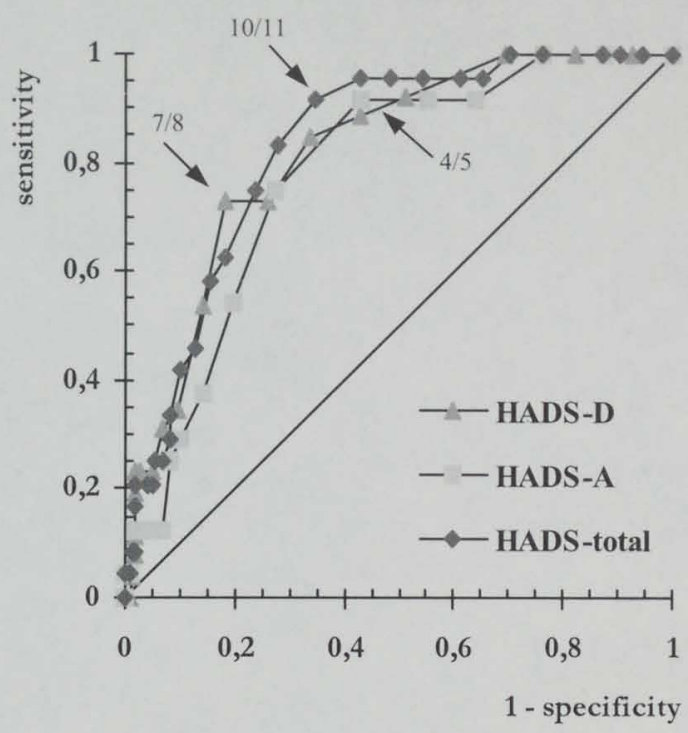

Screening for both major and minor depression did not change the overall accuracy of the instruments, as reflected by the ROC-curves. The optimum cut-off values for the BDI, SCL-90-D and total HADS also remained the same, while for the HAM-D, 11/12 became the best cut-off value. For the HADS-D, however, it decreased by one point to $6 / 7$. The sensitivity and specificity did not change substantially compared to screening for major depression only (Table 2).

Separate ROC analyses for men and women revealed a clear gender effect on the validity of the scales (Figure 3). Especially for the self-rating scales, the AUCs were higher in male than in female respondents (SCL-90-D 0.89 vs. 0.69; BDI 0.86 vs. 0.69 ; HADS-D 0.87 vs. 0.77 ; total HADS 0.94 vs. 0.72 ; HAM-D 0.87 vs. 0.80 ). Optimum cut-off levels also varied between men and women, but this did not substantially improve screening performance.

\section{DISCUSSION}

Our main finding is that all scales are acceptable screening instruments for PSD, but that their performance is clearly worse in women than men. This is best represented by the difference in AUC (ROC analysis), which reached levels as high 
as $34 \%$ (BDI) and $40 \%$ (SCL-90-D) of the maximum possible variation. At optimum cut-off levels, this almost exclusively concerned false-positive cases.

A possible explanation for this sex difference lies in the fact that women tend to report more aspecific complaints of distress than men. ${ }^{140}$ This was confirmed by logistic regression analysis. The significant effect of female sex on a false-positive screening test (OR 2.63; $\mathrm{p}=0.005)$ largely disappeared when the total SCL-90 score was entered into the logistic model as a measure of distress (OR 2.58; $\mathrm{p}=0.07$ ). In

Figure 3. Receiver Operating Characteristics (ROC) curves of the SCL-90, BDI, HADS-D, and HAM-D for men and women separately.

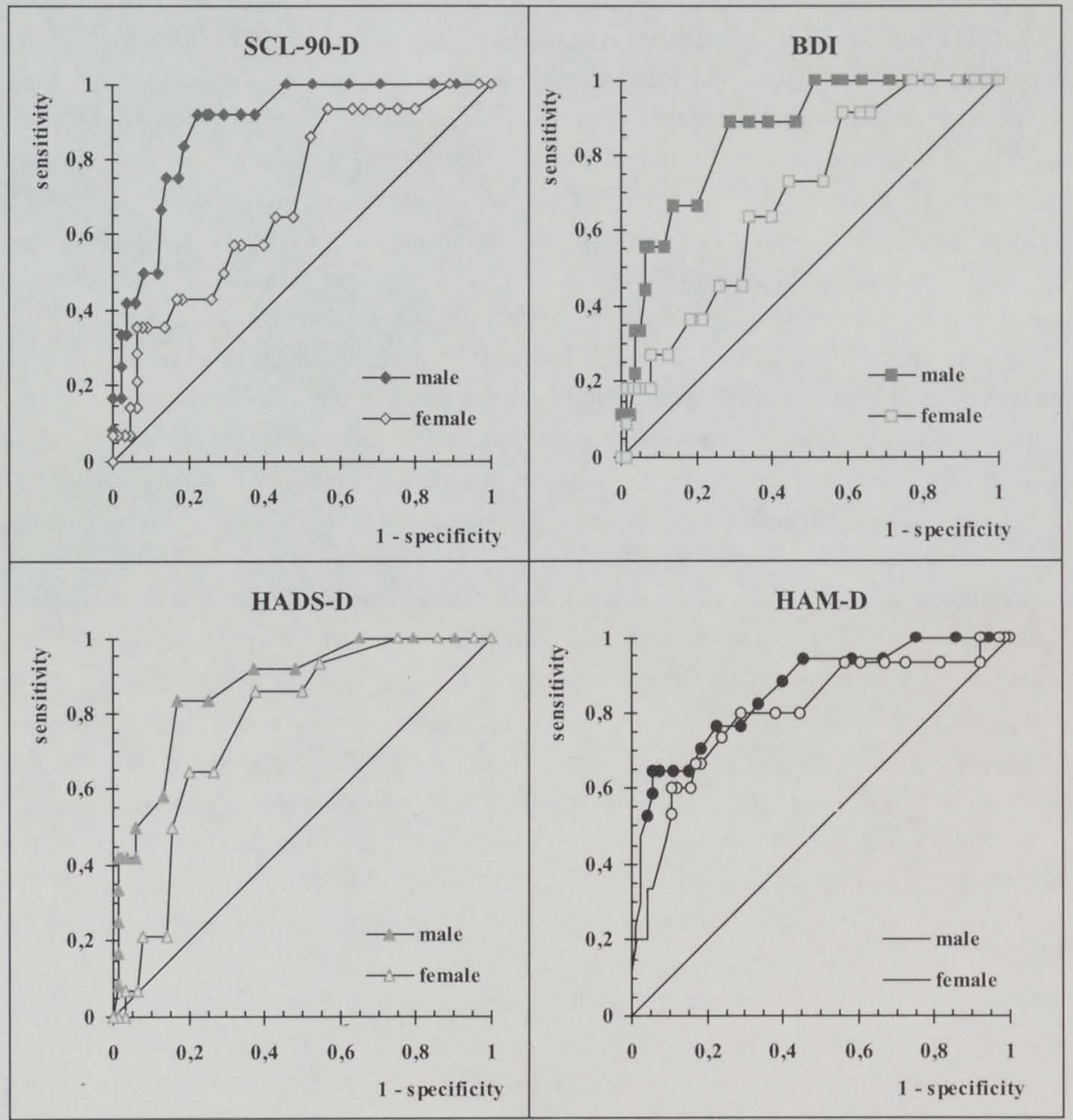


addition, total SCL-90 score proved to be a strong, independent predictor of a false-positive test result (OR 1.11, $\mathrm{p}<0.0001)$. Severity of handicaps (i.e. Rankin score) was also found to have contributed to the sex difference. When entered into the model, it further decreased the independent effect of $\operatorname{sex}(\mathrm{OR} 1.75 ; \mathrm{p}=0.32$ ), while it was independently related to the accuracy of the screening test itself (OR $1.86 ; 0.04)$. No effect was found for age or global cognitive functioning (i.e. MMSE score).

Use of the previously recommended, and generally accepted cut-off levels turned out to be optimal in case of the BDI $(9 / 10)$ and HADS-D (7/8). Defining separate thresholds for men and women did not improve their performance meaningfully. This finding does not support earlier reports on the screening performance of depression-rating scales in stroke patients, indicating that the original cut-off values for the HADS or BDI are sub-optimal in this patient population. $80,81,83$

Overall, the total HADS was just as accurate as the HADS-D, and combined high sensitivity (91.7) with acceptable specificity (65.3) at its optimum cut-off level of 10/11. Even the anxiety subscale alone performed satisfactorily. Indeed, the correlation between HADS-D and HADS-A scores was high $(r=0.67 ; p<0.001)$, which illustrates the frequent coincidence of symptoms of anxiety and depression in stroke patients. Moreover, the total HADS may be even more accurate in detecting cases of post-stroke depression than the depression subscale alone.

The previously used cut-off value of the SCL-90-D combined a relatively low threshold for men (22/23) with a higher level for women (27/28). However, our results indicate that using separate cut-off values for men and women is not clearly superior to using $24 / 25$ as one uniform threshold (sensitivity 88.5 , specificity 60.7 ). The HAM-D had nearly equal sums of sensitivity and specificity at cut-off levels $11 / 12$ and 16/17 for both men and women. This can be explained by the lack of smoothness of the ROC-curves due to the limited sample size. It seems, therefore, quite arbitrary to use separate cut-off values for men and women. Based on our data, it seems most sensible to set the threshold at 11/12 (sensitivity 78.1, specificity 74.6) if the HAM-D is used for screening purposes. For diagnostic purposes, 16/17 (sensitivity 62.5, specificity 91.7 ) would be most favourable, because of the higher specificity (increasing the overall diagnostic accuracy).

Screening for both major and minor depression did not meaningfully decrease sensitivity, nor improve specificity $(\leq 5 \%)$. Optimum thresholds for the SCL-90-D, $\mathrm{BDI}$, total HADS and HAM-D were the same as in screening for major depression alone. For the HADS-D, it was lowered by one point to $6 / 7$.

Lastly, the internal consistency of all three self-rating scales was high and only slightly decreased in a subset of patients with low MMSE scores ( $\leq 23)$. In this subgroup, non-response was clearly higher. Moreover, we cannot exclude that relatives may have helped the patients to complete the scales. 'This suggests that, 
overall, stroke patients are able to fill out these scales reliably, but problems arise in more cognitively impaired patients.

The generalisability of the present findings mainly depends on both the prevalence rate of post-stroke depression in our sample and the main patient characteristics. In our sample, $25.2 \%$ of the patients were diagnosed as suffering from post-stroke depression 1 month after the stroke. This is similar to the prevalence of $20-30 \%$ found in large epidemiologic studies. ${ }^{61,72,94,101,102}$ The age, gender ratio, and demographic characteristics of our sample are representative for a general stroke population. ${ }^{141,142}$ Therefore, our findings can be generalized to other samples of stroke patients, with the exception of severely cognitively damaged patients.

We determined whether misdiagnoses were more likely to occur with increasing delay between the diagnostic interview and completion of the self-rating scales (which were filled out at home afterwards). However, logistic regression analysis showed no significant relationship. Also, misdiagnoses were not significantly more frequent among those 20 patients who fllled out the self-rating scales before the diagnostic interview.

Another potential source of bias lies in the administration of the SCID and HAM$\mathrm{D}$ by the same interviewer during one session. This may have increased the agreement between the two instruments. However, this effect was kept minimal by strictly applying the standard user manuals of both instruments and by strictly separating questions concerning the different instruments. The inconsistent findings of the SCID and the HAM-D can be explained by the fact that the SCID concerns a depressive episode lasting at least 2 weeks in the last month, whereas we used the HAM-D to rate symptoms during the last week only.

Our results indicate that none of the self-rating scales we studied are suitable to diagnose post-stroke depression in clinical settings or to distinguish between depressed and non-depressed subjects in research settings because of the high rate of misdiagnoses of 20 (HADS-D) to almost $40 \%$ (BDI). However, the HAM-D turned out to be diagnostically accurate in $87.1 \%$ of cases at its optimum "diagnostic" cut-off point (16/17). Leentjens et al. found a similar accuracy of the HAM-D in patients with idiopathic Parkinson's disease. ${ }^{14.3}$

As there was no substantial difference in screening performance between the four rating scales, both the total HADS and BDI could be preferred in screening for PSD because they need the least time to administer. Although the observer-based HAM-D has similar predictive validity, it requires trained staff to be administered and may, therefore, be less desirable for screening in day-to-day practice. The relatively high accuracy and specificity of the HAM-D make this instrument more suitable for diagnostic and dichotomization purposes. 


\section{CHAPTER 4}

A comparative study into the 1-year cumulative incidence of depression after stroke and myocardial infarction

This chapter was published as:

Aben I, Verhey F, Strik J, Lousberg R, Lodder J, and Honig A. A comparative study into the 1-year cumulative incidence of depression after stroke and myocardial infarction. Journal of Neurology, Neurosurgery, and Psycbiatry (2003), $74: 581-585$ 


\section{ABSTRACT}

Background The high incidence of post-stroke depression (PSD) has been claimed to reflect a specific, stroke-related pathogenesis, in which cerebral lesion location has been thought to play an important role. By consequence, PSD can be expected to occur more compared to depression in the course of a comparable disease without such cerebrovascular damage.

Aim To evaluate whether depression occurs more frequently after stroke than after myocardial infarction (MI).

Method The 1-year incidence of depression was compared in 190 stroke patients and $200 \mathrm{MI}$ patients. Every three months patients were screened for depressive symptoms, and when positive, assessed by the Structured Clinical Interview of DSM-IV for the existence of major and minor depression.

Results The cumulative 1-year incidence of major and minor depression was similar in stroke and MI patients after controlling for sex, age, and level of handicap.

Conclusions The increased incidence of PSD seems not to reflect a specific cerebrovascular pathogenesis. Further research is needed to investigate whether vascular factors play a common role in the development of depression after stroke and MI. 


\section{INTRODUCTION}

Different pathogenic models have been proposed to explain the high prevalence of PSD. The most influential model suggested a neurobiological mechanism, with the highest risk for depression in cases with a left hemispherical infarction. 20, $11+$ Depression in the elderly has also been related to a more general vascular pathogenesis, being associated with white matter hyperintensities and multiple silent cerebral infarctions on magnetic resonance imaging (MRI). 25, 116 In addition, non-specific mechanisms, such as personality traits ${ }^{1+4}$ and lack of social support ${ }^{145}$, 146 may also be involved in the pathogenesis of PSD.

The few studies that directly compared the prevalence of depression in patients with cerebrovascular disease and non-cerebral vascular disease reported a higher prevalence in stroke patients. ${ }^{147,148}$ However, these studies may be criticised because of cross-sectional designs, small sample sizes, depression diagnoses based on rating scale scores, and lack of control of potential confounders such as age, sex, and severity of handicap.

In this study, we compared the cumulative 1-year incidence of depression after stroke and MI, after controlling for such potential confounders. Like stroke, MI has an acute onset, is life threatening, can recur, and tends to have a chronic course with a mild-to-severe impact on the patient's quality of life. Moreover, stroke and MI share similar vascular risk factors. Therefore, if the incidence of PSD differs from post-MI depression after controlling for sex, age, and level of handicap, it may be seen as circumstantial evidence that this reflects differences in the pathogenesis of depression between these two diseases.

\section{PATIENTS}

One hundred-ninety consecutive patients with a diagnosis of first-ever, hemispheric cerebral infarct and 200 patients with a diagnosis of first-ever MI were included in the study. The patients were recruited from the Emergency Department of the University Hospital of Maastricht, the Netherlands. This University Hospital serves approximately 200,000 inhabitants, being the only hospital in the Maastricht region. The study was a co-operation between the Departments of Psychiatry, Neurology, and Cardiology.

Stroke was diagnosed by a neurologist according to the WHO criteria. ${ }^{149}$ The ischemic nature of stroke was verified by computed tomography (CT). Patients with other types of stroke (e.g., recurrent stroke, haemorrhage, or brain stem infarct) were not included in order to increase the homogeneity of the study groups. Patient data were entered into a prospective stroke registry, which has been described in detail elsewhere. ${ }^{127}$ MI was diagnosed by a cardiologist according to 
the following criteria: clinical picture and electrocardiographic signs typical for an acute MI, and a maximum value of the enzyme aspartate aminotransferase (ASAT) of at least $80 \mathrm{U} / \mathrm{L}$ (twice the upper limit of normal). ${ }^{150}$

Patients from both cohorts were excluded if they had a major psychiatric disorder other than affective disorders (e.g., schizophrenia or a current psychotic episode); if they reported the onset of a current depressive episode before the time of the stroke/MI; if they had a comorbid intracerebral disease (e.g., a brain tumour or Parkinson's disease); or if they were unable to understand the informed consent procedure (e.g., because of severe aphasia or cognitive dysfunction). Concerning the latter, exclusion was based on combined clinical judgement, and Mini Mental. State Examination (MMSE) and Frenchay Aphasia Screening Test (FAST) results (see methods section).

All participants gave written informed consent. The study was approved by the local Medical Ethics Committee.

\section{METHODS}

Optimal comparability was achieved by using a similar study design and similar methods for both patient groups. All patients were followed-up during the first year after stroke or MI. One month following stroke or MI, all patients were interviewed using the Structured Clinical Interview of DSM-IV (SCID-I-R), 132 allowing for a diagnosis of both major and minor depression. ${ }^{47}$ The severity of depressive symptoms was measured with the 17-item Hamilton Depression Rating Scale (HAM-D). ${ }^{96}$ All interviews were administered by well-trained research physicians (IA or JS).

At 3, 6, 9, and 12 months' follow-up, depression was diagnosed according to a twostep procedure. First, patients were administered three psychiatric self-rating scales for depression, namely, the Beck Depression Inventory (BDI), 69,133 the Hospital Anxiety and Depression Scale (HADS), ${ }^{129,} 134$ and the 90-item Symptom Checklist (SCL-90). ${ }^{135,} 136$ Then patients who exceeded the previously defined cut-off value on at least one of these scales were re-interviewed using the SCID and HAM-D. For the BDI, the cut-off value was $9 / 10$, for the HADS $7 / 8$ for both subscales separately, and for the depression subscale of the SCL-90 a threshold of $22 / 23$ was used for men and $27 / 28$ for women. The sensitivity of this screening procedure has previously been proven to be high, being $91.2 \%$ in stroke patients and $85.7 \%$ in MI patients. ${ }^{151}, 152$

At the 1-month follow-up, disability and handicap were rated using the Barthel Index ${ }^{130}$ and Rankin score, ${ }^{131}$ respectively. Data concerning demographics, level of education, living situation, family history of psychiatric disorders, and medication were collected by interview. The personal history of depression was 
measured using the SCID-I-R. In stroke patients, MMSE 128 and FAST ${ }^{153}$ were administered to measure global cognitive functioning and aphasia, respectively.

\section{ANALYSIS}

Cumulative incidence rates for PSD and post-MI depression were analysed using survival analysis techniques. If data on depressive status were missing, patients were considered "not depressed" if they were not depressed at the previous assessment and if the depressive status at the next assessment was validly measured. In all other situations, the case was excluded from further analysis from the time point of the missing value onward. Cox regression was performed with cohort (stroke or MI) as independent variable, controlling for sex, age, and level of handicap (Rankin score). The Rankin scale is actually an ordinal 6-point scale, in which two consecutive scores can be considered to represent equal increases in level of handicap. Therefore, it was allowed as a covariate without dummification. The output of the Cox analysis was checked for instability by influential cases and for violation of both the proportional hazards assumption and the assumption of linearity of effects.

For comparisons between descriptive sample characteristics, Student's t-test was used in the case of continuous, normally distributed variables. Mann-Whitney $U$ test was used for non-normally distributed or ordinal variables. Chi-square test was used for all dichotomous variables. Family history of psychiatric disorders was rated separately for different groups of disorders (affective, schizophrenic, substance abuse, etc) but was dichotomised into positive or negative psychiatric family history in order to optimise statistical power. Lastly, one-way ANOVA was used to compare differences in HAMD scores between major depressed, minor depressed, and non-depressed patients.

For all analyses, the level of statistical significance was set at $\mathrm{p}<0.05$ (two-tailed).

\section{RESULTS}

The stroke patients were selected from a total sample of 444 consecutive patients with a first-ever hemispheric cerebral infarction. One hundred-ninety-three patients $(43.5 \%)$ were excluded, mainly because of severe comorbidity (54 patients [28.0\%] with severe cognitive disorders, including aphasia; 38 [19.7\%] for severe somatic comorbidity, e.g., loss of consciousness, general frailty, and comorbid intracerebral disease; and 12 [6.2\%] for combined cognitive and somatic comorbidity. Further reasons for exclusion were death within 1 month after stroke (37 patients, 19.2\%), current depressive episode at the time of the stroke ( 8 patients, $4.1 \%$ ), and current 
TABLE 1. Reasons for non-response at 3, 6, 9, and 12 months after stroke or myocardial infarction (MI).

\begin{tabular}{llllllllll}
\hline & \multicolumn{4}{c}{$\begin{array}{c}\text { Stroke } \\
\mathbf{n = 1 9 0}\end{array}$} & & & \multicolumn{3}{c}{$\begin{array}{c}\text { MI } \\
\mathbf{n = 2 0 0}\end{array}$} \\
\hline Months post stroke/MI & 3 & 6 & 9 & 12 & 3 & 6 & 9 & 12 \\
\hline Incidental non-response & 2.6 & 1.6 & 1.6 & 0.5 & 2.0 & 0.5 & 0.5 & 1.0 \\
Withdrawn consent & 10.0 & 12.1 & 13.2 & 13.7 & 5.0 & 5.0 & 7.0 & 9.5 \\
Died & 2.1 & 3.7 & 5.3 & 5.3 & 0.5 & 0.5 & 0.5 & 1.0 \\
Incapability & 2.1 & 4.2 & 3.2 & 3.7 & - & - & - & - \\
Lost to follow-up & 0.5 & 0.5 & 0.5 & 0.5 & - & 0.5 & - & - \\
\hline Total & 17.3 & 22.1 & 23.8 & 23.7 & 7.5 & 6.5 & 8.0 & 11.5 \\
\hline
\end{tabular}

Values represent percentages

other major psychiatric disorder (4 patients, 2.1\%). The remaining 40 patients $(20.7 \%)$ were excluded because of other reasons (living outside the Maastricht region, untraceability despite repeated efforts, language barriers, etc). Of the remaining 251 eligible patients, $61(24.3 \%)$ refused participation. Thus, 190 stroke patients remained in the study.

In the MI group, 200 of a total of 415 consecutive patients were included (48\%). Ninety-five MI patients were excluded (22.9\%). Reasons for exclusion were death within the first month post MI (28 patients, 29.5\%), severe comorbidity (19 patients, $20.0 \%$ ), and psychiatric disorder (6 patients [6.3\%] had depressive disorder at the time of the MI, and 3 patients $[3.2 \%]$ had some other major psychiatric disorder). The remaining 39 patients $(41.1 \%)$ were excluded because of reasons such as living outside the Maastricht region, untraceability despite repeated efforts, language barriers, etc. Of the 320 eligible MI patients, $120(37.5 \%)$ refused participation.

Patients who refused to participate were somewhat older than participants in both the stroke cohort $(72.4 \pm 9.7$ vs. $68.6 \pm 11.7 ; \mathrm{t}(2)=2.6, \mathrm{p}=0.01)$ and the MI cohort ( $65.6 \pm 12.2$ vs. $60.1 \pm 10.8 ; \mathrm{t}(2)=4.2, \mathrm{p}<0.001)$. In the case of MI, women refused to participate more often than men did $\left(53.1 \%\right.$ vs. $\left.30.6 \% ; \chi^{2}(1)=14.6 ; p<0.001\right)$. In order to test the assumption that depressive feelings and symptoms are predictive of participation refusal, refusers were asked to fill out the SCL-90 and HADS. In total $57.4 \%$ of the stroke patients who refused participation did so compared with $33.3 \%$ of the MI patients who refused participation. In both cohorts, the mean depression scores of those who refused participation were not significantly different from those who participated. Loss to follow-up occurred more often in the stroke cohort, with $17.4 \%$ non-response at 3 months post stroke and $23.7 \%$ at 12 months post stroke. In the case of the MI cohort, non-response was $7.5 \%$ at 3 
months post stroke and $11.5 \%$ at 12 months post stroke. The reasons for nonresponse are summarised in table 1.

The major characteristics of both study samples are summarised in table 2 . Stroke patients differed from MI patients with regard to age, sex distribution, level of education, and level of handicap. On average, stroke patients were almost 9 years older than MI patients. In the stroke cohort, almost half of the patients were women (46.8\%), while only a quarter of the MI patients were $(23.0 \%)$. Stroke patients were more severely disabled and handicapped.

The 1-year cumulative incidence of depression (major and minor together) was $38.7 \%$ after stroke and $28.4 \%$ after MI (adjusted for cases with incomplete followup; see Table 3). Thus, stroke patients tended to have a higher risk of depression than MI patients did, although this difference was not statistically significant (HR 1.4; $\mathrm{p}=0.06$ ). However, if sex, age, level of handicap (Rankin score), and the interaction between younger age and greater severity of handicap (age*Rankin) were entered into the analysis as covariates, this tendency disappeared (HR 1.1; $\mathrm{p}=0.74)$. Moreover, both male sex and age*Rankin independently predicted depressive outcome (Table 4). Figure 1 shows the cumulative incidence of the

TABLE 2. Demographic and outcome-related characteristics of stroke $(n=190)$ and myocardial infarction (MI; $\mathrm{n}=200$ ) patients

\begin{tabular}{llll}
\hline & $\begin{array}{l}\text { Stroke } \\
\mathbf{n}=190\end{array}$ & $\begin{array}{l}\text { MI } \\
\mathbf{n}=200\end{array}$ & $\mathrm{p}$ \\
\hline Sex (female) & $46.8 \%$ & $23.0 \%$ & $<0.001$ \\
Age, years (mean, SD) & $68.6(11.7)$ & $60.0(10.8)$ & $<0.001$ \\
Level of education & & & $<0.001$ \\
$\quad$ primary school & $42.2 \%$ & $25.9 \%$ & \\
$\quad$ junior secondary vocational cducation & $25.8 \%$ & $19.3 \%$ & \\
$\quad$ senior secondary vocational education & $19.0 \%$ & $31.5 \%$ & \\
$\quad$ (pre-)university education & $13.0 \%$ & $23.3 \%$ & \\
Living alone & $35.7 \%$ & $18.0 \%$ & $<0.001$ \\
MMSE (median, min-max) & $26(16-30)$ & - & - \\
FAST (median, min-max) & $27(9-30)$ & - & - \\
Barthel (mean, s.d.) & $17.4(4.7)$ & $20.0(0.2)$ & $<0.001$ \\
Rankin (mean, s.d.) & $2.4(1.2)$ & $1.2(0.7)$ & $<0.001$ \\
Personal history of depression & $21.8 \%$ & $12.0 \%$ & 0.01 \\
Family history of depression & $20.1 \%$ & $10.0 \%$ & 0.006 \\
Family history of other psychiatric disorders & $43.3 \%$ & $11.5 \%$ & $<0.001$ \\
\hline
\end{tabular}

MMSE: Mini Mental State Examination (minimum score: 0, maximum score: 30); FAST: Frenchay Aphasia Screening Test (minimum score: 0, maximum score: 30); Barthel Index (completely dependent in Activities of Daily Living: 20, no disabilities: 0); Rankin (no handicaps: 0 , bedridden: 5). 
TABLE 3. Cumulative incidence of depression after stroke or myocardial infarction (MI) during 1 year of follow-up (irrespective of the distribution of age, sex, and handicap).

\begin{tabular}{llllll}
\hline & $\begin{array}{l}\text { Time of } \\
\text { assessment }\end{array}$ & $\begin{array}{l}\text { Number } \\
\text { at risk }\end{array}$ & $\begin{array}{l}\text { Incident cases } \\
\text { of depression }\end{array}$ & $\begin{array}{l}\text { Proportion } \\
\text { depressed }\end{array}$ & $\begin{array}{l}\text { Cumulative } \\
\text { proportion } \\
\text { depressed }\end{array}$ \\
\hline & 1 & 190 & 41 & $21.6 \%$ & $21.6 \%$ \\
& 3 & 137.5 & 7 & $5.1 \%$ & $25.6 \%$ \\
STROKE & 6 & 117 & 7 & $6.0 \%$ & $30.0 \%$ \\
& 9 & 107 & 6 & $5.6 \%$ & $33.9 \%$ \\
& 12 & 98 & 7 & $7.1 \%$ & $38.7 \%$ \\
\hline \hline & 1 & 200 & 28 & $14.0 \%$ & $14.0 \%$ \\
MI & 3 & 166 & 9 & $5.4 \%$ & $18.7 \%$ \\
& 6 & 151 & 4 & $2.6 \%$ & $20.8 \%$ \\
& 9 & 146.5 & 11 & $7.5 \%$ & $26.8 \%$ \\
& 12 & 134.5 & 3 & $2.2 \%$ & $28.4 \%$ \\
\hline
\end{tabular}

TABLE 4. Cox regression analysis on the effect of cohort (stroke or myocardial infarction, MI) on the cumulative incidence of depression (major and minor together) with and without controlling for sex and the interaction between age and level of handicap

\begin{tabular}{lllll}
\hline & & HR & 95\%-CI & p \\
\hline BIVARIATE MODEL & stroke cohott & 1.42 & $0.99-2.02$ & 0.06 \\
\hline \hline \multirow{3}{*}{ MULTIVARIATE MODEL } & stroke cohort & 1.08 & $0.70-1.67$ & 0.72 \\
& female sex & 0.62 & $0.42-0.92$ & 0.02 \\
& Age & 1.02 & $0.99-1.06$ & 0.18 \\
& Rankin & 2.05 & $0.99-4.23$ & 0.05 \\
& age x Rankin & 0.99 & $0.97-0.996$ & 0.007 \\
\hline
\end{tabular}

$\mathrm{HR}=$ Hazard Ratio; 95\%-CI $=95 \%$ Confidence Interval; $\mathrm{p}=2$-tailed level of significance; Rankin scores were inverted in order to create an interaction term that increases with increasing age and/or decreasing level of handicap.

controlled analysis. When major depression was analysed separately, its cumulative incidence turned out to be significantly higher after stroke than after $\mathrm{MI}$ on bivariate analysis (HR 2.1; $\mathrm{p}=0.003$ ), but again this difference disappeared after controlling for sex and age*Rankin (HR 1.36; $\mathrm{p}=0.31$ ).

In both cohorts about half of the incident cases of depression had already developed by 1 month (50.7\% of depressed stroke patients and $46.7 \%$ of depressed MI patients; Table 3), indicating that depression had an early onset in many 
patients. In the stroke cohort, 41 patients (23.3\%) met DSM-IV criteria for major depressive disorder and $27(15.4 \%)$ met criteria for minor depressive disorder. In the MI cohort, 23 patients $(11.9 \%)$ were diagnosed with major depression and 32 $(16.5 \%)$ with minor depression. No difference in the severity of depressive symptoms was found between stroke and MI patients, nor did the difference in HAMD scores for major, minor, and non-depressed patients differ between the stroke and MI cohorts (Figure 2).

Of the patients who were considered not depressed according to our assessment, a few were prescribed antidepressive medication by their medical doctor at some time during follow-up ( $7.6 \%$ of stroke patients and $6.5 \%$ of MI patients).

\section{DISCUSSION}

To our knowledge, this study is the first to compare directly the cumulative 1-year incidence of major and minor depression in, stroke and MI patients using a similar longitudinal study design, setting, and methods. Almost $40 \%$ of stroke patients and almost 30\% of MI patients were diagnosed as suffering from major or minor depression during the 1-year follow-up. After we controlled for differences in age, sex, and level of handicap between stroke and MI patients, there was no longer a statistically significant difference in the incidence of depression between the two cohorts. Moreover, in both groups the highest incidence of depression was found in the early phase (i.e. 1 month) after stroke or MI.

At first sight, the results of our study support the general impression from the literature that the incidence of depression is higher in stroke patients than in patients with other vascular diseases. Our data do not, however, favour the hypothesis of a specific pathogenic role of cerebrovascular factors, since the incidence of depression after stroke and MI did not differ after we controlled for confounding variables. Therefore, the increased incidence of depression after stroke and MI may be due to factors that both conditions share. One possible mechanism is that generalised damage to the vascular system in the brain may subsequently affect mood regulatory processes, the so-called vascular hypothesis. ${ }^{25}$, 116 We could not test this hypothesis because CT or MRI data on vascular brain damage were not available for the MI cohort. At present, there is only indirect evidence to support this hypothesis. Several determinants of vascular diseases, such as hypertension, elevated cholesterol levels, and diabetes mellitus, are common to both stroke and MI patients and all have been related to depression. ${ }^{117,} 118$ Damage to the small vessels of the brain, measured as white matter hyperintensities on MRI, 122 and silent cerebral infarctions 119,120 have also been related to depression. However, because patients do not experience symptoms in relation to 
Figure 1. Cumulative 1-year incidence of depression after stroke or myocardial infarction (MI) at means of covariates (age, sex, level of handicap, and age*level of handicap). Hazard ratio for cohort (stroke vs. $\mathrm{MI})=1.08, \mathrm{p}=0.72$.

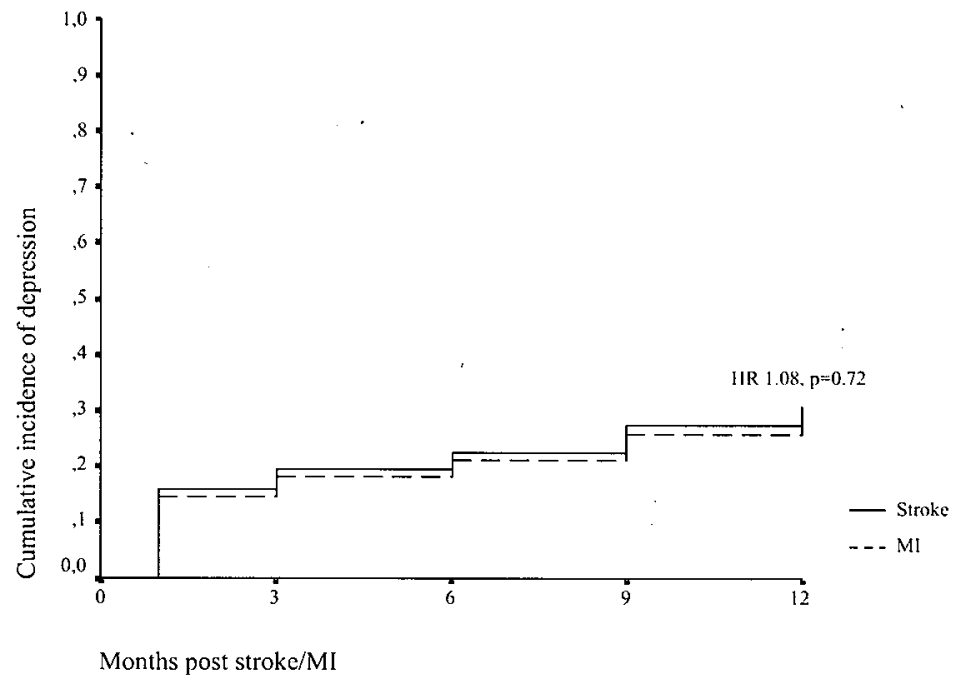

FIGURE 2. Hamilton Depression scores for incident cases of major and minor depression and of non depressed cases in the first year after stroke or myocardial infarction (MI).

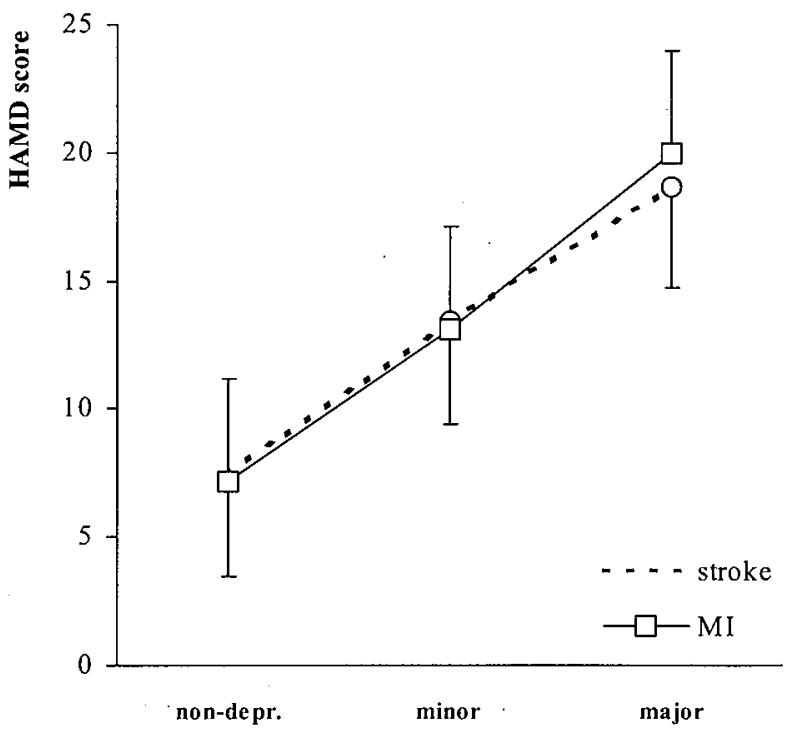


these conditions (except for diabetes), the psychological reaction model cannot explain the high rate of depression.

A strong point of this study is that both cohorts consisted of consecutive series of patients presenting with a first-ever ischemic and hemispheric stroke or a first-ever MI and that depression was measured in a standardised way using uniform methods in both patient groups. A drawback is that patients with severe cognitive dysfunction (dementia, aphasia) or general frailty were excluded. In the stroke cohort in particular, this led to under-representation of patients with severe disabilities. Since we found a more severe level of handicap to be predictive of depression, we cannot rule out that the true incidence of PSD is higher. In addition, the prescription of antidepressive drugs to patients who did not meet criteria for major or minor depression may also indicate that the true incidence is higher than we found. However, certain antidepressant drugs are sometimes prescribed for reasons other than depressive disorder (e.g. neurological pain syndromes, social phobia, obsessive-compulsive disorder), which weakens the assumption that all patients who were prescribed these drugs indeed suffered from a depressive episode.

In conclusion, we found no evidence that the incidence of depression is higher in stroke patients than it is in patients with non-cerebrovascular disease, after taking confounding factors, such as age, sex, and level of handicap, into account. This challenges the hypothesis that specific cerebral factors play a major role in the pathogenesis of PSD. 


\section{CHAPTER 5}

Personality and vulnerability to depression in stroke patients: a 1-year prospective follow-up study

This chapter was published as:

Aben I, Denollet J, Lousberg R, Verhey F, Wojciechowski F, and Honig A. Personality and vulnerability to depression in stroke patients: a 1-year prospective follow-up study. Stroke (2002), 33:2391-2395 


\begin{abstract}
Background and purpose Depression is a frequent sequel of stroke that negatively interferes with rehabilitation outcome. Personality traits have been neglected as potential vulnerability factors for post-stroke depression (PSD). In a 1-year prospective study, the influence of the five main personality traits (i.e. neuroticism, extraversion, openness, agreeableness, and conscientiousness) on the development of PSD was studied.
\end{abstract}

Methods One month after stroke, hundred-ninety consecutive patients with a firstever supratentorial infarct were asked to complete a personality inventory, the NEO-FFI, which is based on the Five Factor Model of personality. Depressive symptoms were assessed 1, 3, 6, 9, and 12 months after stroke, using three selfrating questionnaires as screening instrument for depression. PSD was diagnosed as major or minor depression using the Structured Clinical Interview for DSM-IV.

Results The 1-year cumulative incidence of depression was 38.7\%. Cox regression analysis showed that patients with high neuroticism scores had 4.6 times higher risk of developing PSD than patients with low neuroticism scores $(p=0.001)$ irrespective of lesion location. Level of handicap was the only other factor that showed an independent effect on the occurrence of PSD.

Conclusions Neuroticism is an important predictor of PSD, a finding that emphasizes the need to take personality into account as a potential vulnerability factor for depression in stroke patients. Research on PSD should aim at delineating the interplay between neurological and psychological factors in the development of PSD. 


\section{INTRODUCTION}

Research into determinants of post-stroke depression (PSD) has to a large extent focused on specific biological stroke-related factors, in particular lesion location. ${ }^{20}$ Although recent systematic reviews did not support the hypothesis that left hemisphere strokes increase the risk for PSD, ${ }^{22,}, 23$ Vataja et al. (2001) reported new evidence from a large MRI-based study $(n=275)$, showing that lesions affecting the prefrontosubcortical circuits, especially on the left side, are associated with PSD. ${ }^{21}$

Recent evidence also suggests that, in addition to (neuro)biological factors, psychological factors deserve further exploration. With reference to a recent publication on the effect of psychological distress on the risk of suffering a fatal stroke, ${ }^{154}$ Carney (2002) discussed several mechanisms on how psychological distress and stroke are related. ${ }^{155} \mathrm{He}$ suggested that psychological distress, a collective noun for a number of negative mood states, might rather interact with cerebrovascular disease in determining the course and prognosis of stroke than be the primary causal factor in the pathogenesis of (fatal) stroke. Analogous to this, the tendency to experience negative mood states, in psychological terms called neuroticism ${ }^{156}$ or negative affectivity, ${ }^{157}$ may interact with stroke, thereby facilitating PSD to occur. In one cross-sectional study including 90 patients, Morris and Robinson (1992 \& 1995) linked neuroticism to PSD using a shortened version of the Neuroticism Inventory of Eysenck. ${ }^{110,144}$ However, personality traits as risk factors for PSD should preferably be studied prospectively in a large cohort and with a general assessment of personality traits.

In the present prospective study, a cohort of 190 patients was followed up during one year after a first-ever ischemic stroke. Personality was assessed one month after stroke using the NEO-Five Factor Inventory (NEO-FFI). ${ }^{156}$ The purpose of the study was to examine whether specific personality traits are a risk factor for PSD.

\section{PATIENTS}

Between September 1, 1997 and September 1, 1999, 444 consecutive patients were diagnosed with a first-ever supratentorial infarct at the Emergency Department and the Ambulatory Neurology Clinic of the University Hospital of Maastricht, the Netherlands. This University Hospital serves approximately 200,000 inhabitants, being the only hospital in the region.

Stroke was diagnosed by a neurologist according to the WHO criteria. ${ }^{1+9}$ The ischemic nature of stroke was verified by computed tomography (CT). Differentiation between left and right-sided stroke was based on CT data and/or clinical presentation. 
Hundred-ninety-three (43.5\%) patients were excluded, mainly because of severe comorbidity. Exclusion of patients who were unable to communicate reliably (e.g., because of severe aphasia or cognitive dysfunction) was based on combined clinical judgment and Mini Mental State Examination (MMSE) and Frenchay Aphasia Screening Test (FAST) results (see Methods section). Reasons for exclusion are shown in figure 1.

Sixty-one of the remaining 251 eligible stroke patients refused participation $(24.3 \%)$. Refusers were somewhat older than participants (72.4 \pm 9.7 vs. $68.6 \pm 11.7$ years; $\mathrm{t}(2)=2.6, \mathrm{p}=0.01)$. No sex difference was found between these two groups. Moreover, 35 refusers $(57.4 \%)$ who were willing to fill-out two psychiatric selfrating scales (SCL-90 and HADS, see below), did not report significantly more depressive symptoms than participants.

Thus, 190 stroke patients participated in the study. Major characteristics of this cohort are summarized in table 1. All participants gave written informed consent. The study was approved by the Medical Ethics Committee of the University Hospital Maastricht.

TABLE 1. Demographic and outcome-related characteristics of stroke patients included in the study $(\mathrm{n}=190)$

\begin{tabular}{ll}
\hline & $\mathbf{n}=\mathbf{1 9 0}$ \\
\hline Sex (female) & $46.8 \%$ \\
Age, yrs (mean \pm SD; range) & $68.6 \pm 11.7 ; 36-89$ \\
Level of education & \\
$\quad$ primary school & $42.2 \%$ \\
$\quad$ junior secondary vocational education & $25.8 \%$ \\
$\quad$ senior secondary vocational education & $19.0 \%$ \\
$\quad$ (pre-)university education & $13.0 \%$ \\
Living alone & $35.7 \%$ \\
Stroke lesion location & \\
$\quad$ left & $46.8 \%$ \\
right & $53.2 \%$ \\
MMSE (median, min-max; $\% \leq 23)$ & $26(16-30) ; 21.5 \%$ \\
FAST (median, min-max) & $27(9-30)$ \\
Barthel (mean, s.d.; median) & $17.4(4.7) ; 20$ \\
Rankin (mean, s.d.; median) & $2.4(1.2) ; 2$ \\
Personal history of depression & $21.8 \%$ \\
Family history of depression & $20.1 \%$ \\
Family history of other psychiatric disorders & $43.3 \%$ \\
\hline
\end{tabular}

MMSE: Mini Mental State Examination (minimum score: 0, maximum score: 30); FAST: Frenchay Aphasia Screening Test (minimum score: 0, maximum score: 30); Barthel Index (completely dependent in Activities of Daily Living: 20, no disabilities: 0); Rankin (no handicaps: 0 , bedridden: 5 ) 
FiguRE 1. Patient selection and recruitment.

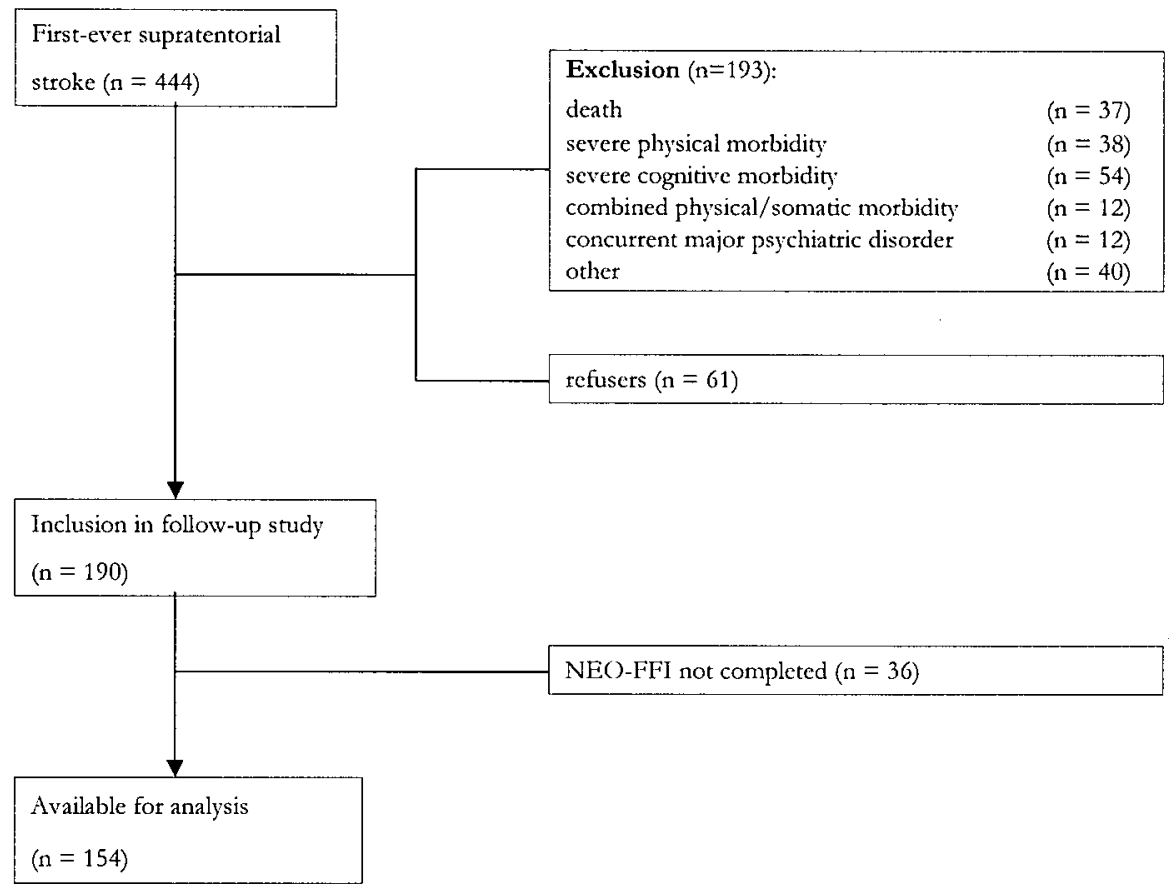

\section{METHODS}

\section{INITIAL ASSESSMENT OF DEPRESSION}

All patients were followed up during the first year after stroke. PSD was defined as an episode of major or minor depression according to DSM-IV criteria ${ }^{47}$ on at least one assessment during the 1 -year follow-up period. After 1 month, all patients were interviewed using both the depression section of the Structured Clinical Interview for DSM-IV (SCID-I-R) ${ }^{158}$ and the Hamilton Depression Rating Scale (HAM-D). ${ }^{96}$ The SCID is a structured psychiatric diagnostic interview allowing for a DSM-IV diagnosis of major or minor depression. The HAM-D is a clinical rating scale that measures the severity of depressive symptoms. All interviews were administered by the same clinician (IA), who was trained to use these instruments. No formal test of inter-rater reliability was performed. 


\section{FOLLOW-UP ASSESSMENT OF DEPRESSION}

At 3, 6, 9, and 12 months after stroke, patients were asked to complete three psychiatric self-rating scales to screen for depression. These were the Beck Depression Inventory (BDI), the Hospital Anxiety and Depression Scale (HADS), and the 90-item Symptom Check List (SCL-90). ${ }^{69,129,135}$ Cut-off levels were 9/10 for the BDI and $7 / 8$ for both the depression and the anxiety subscale of the HADS. In the case of the depression subscale of the SCL-90, the threshold was $22 / 23$ for men and $27 / 28$ for women. The predictive validity of these instruments in this cohort was analyzed previously. ${ }^{151}$ The sensitivity of this screening procedure was shown to be $93.8 \%$ at the 1 -month assessment.

Patients whose scores exceeded the cut-off value for at least one of the self-rating scales were reinterviewed using the SCID and HAM-D in order to diagnose major or minor depression. In $50 \%$ of these cases, the interview was administered within two weeks; in 75\% within 3-3.5 weeks.

In 18 patients the follow-up assessment of depression was incomplete, while the event of interest (depression) had not yet occurred. Of these, 7 withdrew their consent, 4 died, 4 had too severe comorbidity, and 3 did not respond at the final assessment (12 months).

\section{Personality}

Personality was assessed 1 month after stroke, using the NEO Five Factor Inventory (NEO-FFI), ${ }^{156}$ which has been translated into Dutch. ${ }^{159}$ This self-report questionnaire consists of 60 statements covering the five main dimensions of personality: neuroticism, extraversion, openness to new experiences, agreeableness, and conscientiousness. Neuroticism has been related to depression most frequently ${ }^{160}$ and is defined as a stable disposition to experience psychological distress across time and situations consisting of negative emotions such as fear, anger, and frustration. ${ }^{156}$ Each statement is rated on a 5-point scale ranging from 'strongly disagree' to 'strongly agree', resulting in total dimension scores between 12 and 60 .

Non-response concerning the assessment of personality occurred in 36 patients $(18.9 \%)$, either because of study withdrawal or difficulty completing the NEO-FFI.

\section{LEVEL OF DISABILITY}

The MMSE ${ }^{128}$ and FAST $^{153}$ were administered 1 month after stroke to measure global cognitive functioning. At the same time, the level of disability and handicap were rated using the Barthel Index ${ }^{130}$ and Rankin score, ${ }^{131}$ respectively. Data concerning demographics, level of education, living situation, and family history of psychiatric disorders were also collected. Personal history of depression was measured using the SCID-I-R. 


\section{ANALYSIS}

Cox regression was used to analyze the relative hazard of increasing scores for the five dimensions of personality (as measured with the NEO-FFI) on the incidence of PSD (major and minor depression combined). Personality traits were entered into the analysis as independent variables and sex, age, history of depressive disorder, and level of handicap (Rankin score) were added as potential confounders. Both forward and backward stepwise modelling procedures were applied to optimize the model. The likelihood-ratio statistic based on the maximum partial likelihood estimate was used for variable entry and removal. The criterion for entry or removal was set at $\mathrm{p}<0.05$. The output of the Cox analyses was checked for instability by influential cases and for violation of both the proportional hazards assumption and the assumption of linearity of effects.

Post hoc, SCL-90 total scores for the 1-month assessment were entered into the optimized model as a measure of distress. While neuroticism refers to a stable disposition to experience psychological distress across time and situations, distress refers to the momentary level of distress and is more dependent on situational characteristics. In additional post hoc analyses, incident cases of depression 1month post stroke were excluded from the analysis to ensure that the effects were not due to depression affecting the personality assessment. Potential effects of side of stroke lesion (left versus right hemisphere) and sex on associations between personality dimensions and depression were also tested.

For group comparisons of descriptive sample characteristics, Student's t-test was used in the case of continuous normally distributed variables. Chi-square test was used for all dichotomous variables. Level of significance was set at $\mathrm{p}<0.05$ (2tailed) for all analyses. Where appropriate, results are given as means $\pm \mathrm{SD}$.

\section{RESULTS}

\section{PERSONALITY TRAITS AND DEPRESSION}

The 1-year cumulative incidence of depression was 38.7\% (adjusted for cases with incomplete follow-up). Cross-sectionally, the incidence rates were $21.6 \%(41 / 190)$ at 1 month, $5.1 \%(7 / 137.5)$ at 3 months, $6.0 \%(7 / 117)$ at 6 months, $5.6 \%(6 / 107)$ at 9 months, and $7.1 \%(7 / 98)$ at 12 months. Of these, 41 patients $(23.3 \%)$ met DSM-IV criteria for major depressive disorder and 27 (15.4\%) met criteria for minor depressive disorder. The mean HAMD score was $19.2 \pm 4.1$ for the patients with major depression, $13.2 \pm 4.3$ for the patients with minor depression, and $7.3 \pm$ 4.1 for the non-depressed patients. These differences were statistically significant $(\mathrm{F}(2)=198.2 ; \mathrm{p}<0.001)$.

Mean personality domain scores measured 1-month after stroke, as well as the concordant hazard ratios (HR) from bivariate Cox regression analyses, indicating 
TABle 2. Mean personality domain scores (NEO-FFI) and Hazard Ratios from bivariate Cox regression ( $n=154)$.

\begin{tabular}{llllll}
\hline & Neuroticism & Extraversion Openness & Altruism & $\begin{array}{l}\text { Conscienti- } \\
\text { ousness }\end{array}$ \\
\hline major depression & $33.5 \pm 7.3$ & $37.6 \pm 6.5$ & $31.2 \pm 6.3$ & $44.5 \pm 5.7$ & $44.8 \pm 5.9$ \\
minor depression & $33.3 \pm 6.7$ & $37.3 \pm 7.6$ & $33.5 \pm 6.0$ & $45.0 \pm 3.4$ & $45.1 \pm 4.7$ \\
no depression & $28.3 \pm 6.4$ & $40.1 \pm 4.8$ & $33.6 \pm 6.1$ & $43.5 \pm 5.0$ & $45.5 \pm 5.3$ \\
censored & $28.2 \pm 8.6$ & $38.1 \pm 5.3$ & $30.3 \pm 6.8$ & $46.5 \pm 5.8$ & $45.7 \pm 5.6$ \\
\hline HR (p-value) & $1.08(0.0001)$ & $0.94(0.01)$ & $0.98(0.36)$ & $1.02(0.41)$ & $0.98(0.50)$ \\
\hline
\end{tabular}

HR = Hazard Ratio.

the relative risk to develop depression at any time during the 1-year follow-up, are shown in table 2. Neuroticism scores ranged from 12 to 46 (30.1 \pm 7.3$)$. On average, depressed patients (both major and minor) scored 5 points higher on the neuroticism scale than non-depressed patients or patients with incomplete followup (censored cases). On bivariate Cox regression analyses, each point increase in neuroticism score increased the relative hazard (HR) for PSD (major and minor depression combined) by $8 \%(\mathrm{p}=0.0001)$. This means that a patient who scored 1 standard deviation above the mean had a relative risk of 1.75 of developing PSD compared to someone with an average neuroticism score.

Depressed patients had scores for extraversion about 3 points lower than those of non-depressed patients. Overall, scores on this scale ranged from 20 to 54 (Mean $38.9 \pm 5.8$ ). For each 1-point lower score for extraversion, the HR for PSD increased by $6 \%(\mathrm{p}=0.005)$. The other domains of the NEO-FFI did not show significant bivariate effects on the risk of PSD.

\section{MULTIVARIATE CoX REGRESSION ANALYSIS}

If all domains of the NEO-FFI were entered into the analysis together with the controlling variables sex, age, history of depression, and level of handicap (Rankin), the effect of a high level of neuroticism remained statistically significant ( $p=$ 0.006), whereas the effect of a low level of extraversion disappeared (Table 3). Level of handicap was the only covariate that showed an independent significant effect $(p=0.03)$. Both forward and backward stepwise modelling resulted in the same model involving only neuroticism and level of handicap (Table 3 ).

In addition, the above-described model was re-analyzed using three categories for both neuroticism and Rankin scale scores. In order to create categories of similar size, the tertiles of the observed values were used as cut-off points. In the case of neuroticism this resulted in: low ( $\leq 26)$, intermediate (27-33), and high $(\geq 34)$. In 
the case of Rankin, this resulted in: $0-1,2-3$, and 4-5. For convenience, this model was used to show the effect of neuroticism in figure 2 .

Entry of the total SCL-90 score as a measure of 'distress' into the analysis did not diminish the effect of neuroticism, as measured with the NEO-FFI $(p=0.02)$, neither did removal of those patients who already suffered from depression at 1 month after stroke when the NEO-FFI was administered $(\mathrm{p}=0.007)$.

\section{POST-HOC ANALYSIS: EFFECT OF LESION LOCATION, SEX, AND MMSE}

Eighty-nine patients (46,8\%) had a left-sided stroke and 101 patients $(53.2 \%)$ had a right-sided stroke. PSD was not more frequent in left-sided strokes (45.7\%) than in right-sided strokes $(41.4 \% ; \chi 2(1)=0.3 ; \mathrm{p}=0.59)$ and lesion location was not related to personality domain scores ( $p>0.6$ for each of the 5 NEO-FFI domains). Most importantly, the side of the stroke lesion (left vs. right hemisphere) did not change the risk-increasing effect of neuroticism on PSD.

Further post-hoc analysis, to see whether patient sex influenced the relationship between high level of neuroticism and depression, revealed an interaction effect after removal of three outliers (HR 0.90, $\mathrm{p}=0.03$ ) and showed that the effect of neuroticism was stronger in men than in women.

TABLE 3. Multivariate Cox regression analysis of the five personality traits of the NEO-FFI as risk factor for post-stroke depression at any time point during the first year after stroke, controlling for age, sex, personal history of depression, and level of handicap $(\mathrm{n}=154)$.

\begin{tabular}{llll}
\hline & HR & $95 \%$ CI & P \\
\hline FULL MODEL & & & \\
Neuroticism & 1.08 & $1.03-1.13$ & 0.003 \\
Extraversion & 0.98 & $0.93-1.04$ & 0.56 \\
Openness & 0.99 & $0.95-1.04$ & 0.79 \\
Altruism & 1.04 & $0.98-1.10$ & 0.24 \\
Conscientiousness & 1.02 & $0.97-1.08$ & 0.51 \\
Age & 1.00 & $0.97-1.02$ & 0.80 \\
Sex & 1.23 & $0.69-2.22$ & 0.48 \\
History of depression & 1.50 & $0.76-2.93$ & 0.24 \\
Level of handicap & 1.36 & $1.03-1.79$ & 0.03 \\
\hline STEPWISE OBTAINED MODEL & & & \\
Neuroticism & 1.08 & $1.04-1.12$ & 0.0001 \\
Level of handicap & 1.36 & $1.08-1.72$ & 0.01 \\
\hline
\end{tabular}

HR indicates the relative risk increasing effect for each 1 point increase in the value of the covariate. 
FIGURE 2. Cumulative 1-year incidence of post-stroke depression in patients with low, intermediate, and high neuroticism scores (categories based on tertiles). HR = Hazard Ratio (with low neuroticism as the reference group)

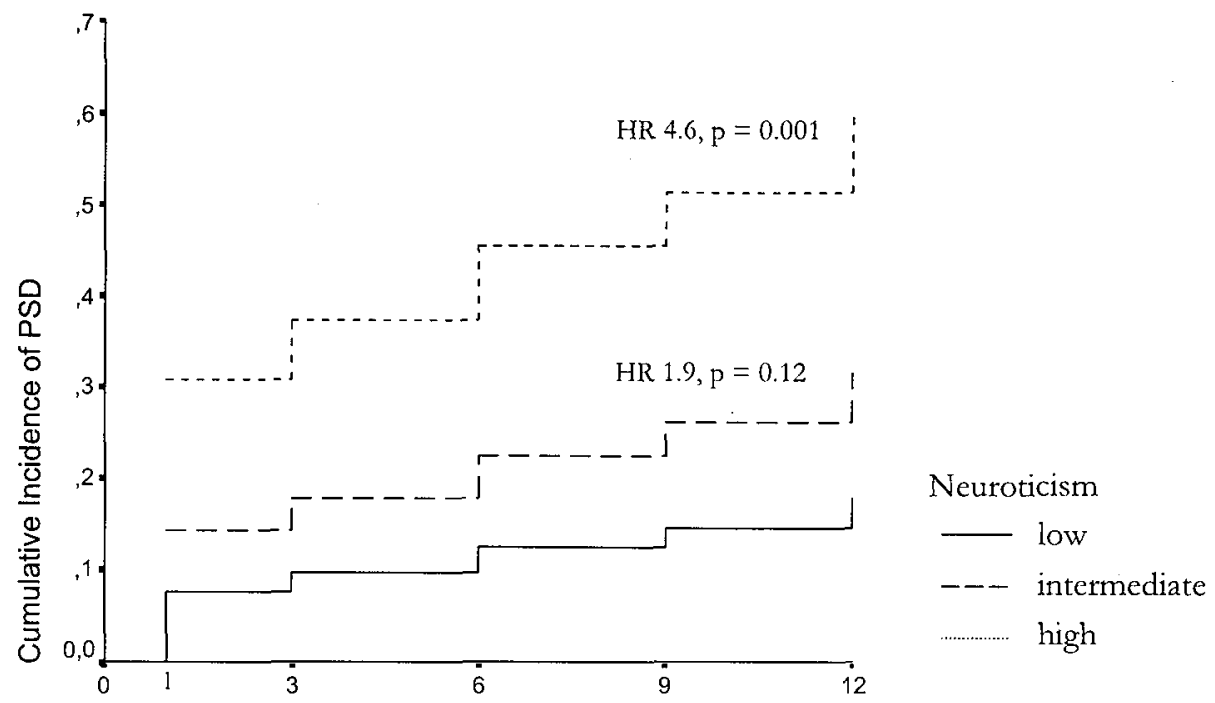

months after stroke

Lastly, no significant relation was found between MMSE scores and PSD ( $\mathrm{t}(134)=$ 1.1 ; p. 0.29 ). Higher MMSE scores were associated with more extraversion and openness to experience, and with less neuroticism, agreeableness, and conscientiousness $\left(\mathrm{r}^{2}=0.15-0.23, \mathrm{p}=0.06-0.004\right)$.

\section{DISCUSSION}

This study showed that neuroticism is a vulnerability factor for depression in the first year after a first-ever cerebral infarct. For each point increase in neuroticism, the risk of depression increased by a factor of 1.08 . Moreover, our data indicated that this effect was stronger in men than women. Apart from neuroticism, no other dimension of the Five Factor Model of personality showed an independent significant effect on the development of PSD.

The findings of our study are important from both a clinical and a theoretical perspective. Personality assessment may help to detect patients at risk of PSD early after stroke. Moreover, our findings support the notion that, apart from anatomical factors, PSD also involves psychological factors. On the one hand, the study by 
Vataja et al. (2001) revealed new evidence for lesions affecting the prefrontosubcortical circuits to increase the risk of PSD.21 On the other hand, stroke should also be considered as a negative life-event to which patients may respond with depression depending on the interaction between personality factors and the severity of the negative physical, psychological, and social consequences of stroke. ${ }^{161}$ To determine which factors are independent predictors of PSD, further studies are needed in which neuroticism is taken into account as an important potential interactor or confounder.

A first limitation of our study is that we assessed personality 1 month after stroke. Since stroke is known to potentially cause personality changes, our results may not exclusively reflect premorbid personality traits. However, after controlling for 'distress', as measured with the total score on the SCL-90, the trait neuroticism effect of the NEO-FFI remained statistically significant. Moreover, the finding that the neuroticism effect found in our study proved to be linear contradicts the suggestion that the effect is caused by a subset of patients with stroke-related personality changes based on the location of the stroke lesion. In this case, a more logistic distribution ( $\int$-form curve) would probably have appeared. As a consequence, we believe that our findings reflect the effects of premorbid personality traits on the development of PSD.

A second limitation is that a substantial percentage of PSD patients were diagnosed with depression at the time of personality assessment. It has been reported that depression affects self-rated personality scores toward more pathology, ${ }^{162}$ although this has been contradicted by Bagby. 163 The effect of neuroticism in our study, however, remained significant after the exclusion of patients who were already depressed 1 month after stroke.

Thirdly, a large percentage of patients were excluded from this study, mainly because of severe handicap and/or co-morbidity. This limits the generalisability of our findings to stroke patients with a first-ever hemispheric infarct who survive from stroke without such severe physical or cognitive disabilities that cognitive, psychological and psychiatric evaluations are seriously hampered. In general, diagnosing depression in stroke patients is complicated by several different factors including specific consequences of stroke (e.g. aphasia, anosognosia, denial, and emotional lability) and the high incidence of aspecific symptoms that might and might not be caused by depression (e.g. fatigue, motor slowness, and difficulty concentrating). ${ }^{161}$

The findings of the present study emphasize the need to take personality into account as a potential determinant of depression in patients recovering from stroke. Hence, future research should aim at delineating the interplay between neurological and psychological factors in the development of PSD. 


\section{CHAPTER 6}

Focal or generalised vascular brain damage and vulnerability to depression after stroke:

a 1-year prospective follow-up study

This chapter is submitted for publication as.

Aben I, Verhey F, Boreas A, Lousberg R, Honig A, and Lodder J. Focal or generalised vascular brain damage and vulnerability to depression after stroke: a 1year prospective follow-up study (submitted) 


\section{ABSTRACT}

Background: Both the lesion location hypothesis and the vascular depression hypothesis have been proposed to explain the high incidence of depression in stroke patients. However, research studying the independent contribution of focal versus generalised vascular brain damage in a single cohort of stroke patients is, at present, scarce.

Objective: To test the independent effects of lesion location (left hemisphere, anterior region) and of co-occurring generalised vascular damage on the development of depression in the first year after ischemic stroke, while other tisk factors for depression are controlled for.

Methods: Hundred-ninety consecutive patients with a first-ever, supratentorial infarct were followed up for one year. CT was made in the acute phase of stroke, while in 75 patients an additional MRI scan was also available. Stroke lesion location characteristics and generalised vascular damage were scored using these scans. Depression self-rating scales were used to detect patients with depressive symptoms. Major and minor depression were assessed at 1, 3, 6, 9, and 12 months after stroke according to DSM-IV criteria, using the SCID-I. Additional non stroke-specific risk factors were also measured: sex, age, personal or family history of depression, level of handicap, and level of neuroticism.

Results: Separate analyses of the lesion location hypothesis and the vascular depression hypothesis failed to reveal significant support for either of these biological models of post-stroke depression. Similar negative results appeared from one overall, multivariate analysis including variables of both focal and generalised vascular brain damage, as well as other non-cerebral risk factors. A protective effect of stroke lesions that involved the anterior area of the brain came out of this multivariate analysis. In addition, level of handicap and neuroticism were independent predictors of depression in this cohort, as has been reported previously.

Conclusion: This study does neither support the lesion location nor the vascular depression hypothesis of post-stroke depression. A bio psychosocial model including both premorbid (prior to stroke) vulnerability factors, such as neuroticism and (family) history of depression, as well as post-stroke stressors, such as level of handicap, may be more appropriate and deserves further study. 


\section{INTRODUCTION}

During the last two decades an extensive amount of research has tried to identify the etiological role of cerebral damage in the development of depression in stroke patients. ${ }^{161}$ Most attention has been paid to the 'lesion location hypothesis', which suggests that post-stroke depression (PSD) is directly caused by focal damage to brain regions involved in the mood regulatory system. As an alternative, the socalled 'vascular depression hypothesis' emphasizes the role of generalised vascular brain damage in the development of depression in elderly patients.

Robinson was the first to report that left hemisphere lesions and lesions located to the vicinity of the frontal pole were more frequently associated with PSD than lesions elsewhere in the brain..$^{20}$ Lateralised differentiation of the organisation of emotions, the importance of frontal structures in the regulation of emotional behaviour, and the characteristic distribution of noradrenergic axons via the white matter of the frontal lobes were proposed to underlie these preferential lesion locations in PSD. However, two recent systematic reviews on the relationship between the side of stroke and depression failed to confirm the association between left sided strokes and depression. ${ }^{22,}{ }^{23}$ In a reaction to these publications, a meta-analysis was carried out, which suggested that in left hemisphere stroke, PSD is related to the vicinity of the stroke lesion towards the frontal pole, whereas in right hemisphere stroke this is not the case. ${ }^{164}$

The vascular depression hypothesis has been postulated since different vascular diseases (stroke, coronary artery disease, myocardial infarction, diabetes, etcetera) as well as vascular risk factors are associated with depression. ${ }^{16}$ Krishnan was the first to present evidence that depression in patients with white matter lesions on MRI is characterised by older age, later age at onset of depressive disorder, and a different symptom profile (less feelings of guilt, more anhedonia and motor retardation)..$^{25}$ It may be considered an extension of the lesion location hypothesis, emphasizing that not only single lesions but also an accumulation of (smaller) lesions may induce depression. However, in their cohort of 275 stroke patients, Vataja et al. did not find a risk increasing effect of white matter lesions on the incidence of PSD. ${ }^{21}$

While the evidence for specific biological models for PSD is still conflicting, other factors that need not specifically be stroke-related, should be taken into account. This is in line with the observation that the incidence of depression in the course of other non-cerebral diseases is also increased (MI, rheumatoid arthritis, cancer). ${ }^{165}$, 166 In all these conditions, premorbid vulnerability to depression, dysfunctional coping skills and personality traits, reduced quality of life with disabilities and handicaps, and lack of social support may all contribute to the development of depression. Consistent with such a bio psychosocial model of depression, we have recently shown that the personality trait of neuroticism and the extent to which 
TABLE 1. Demographic and outcome-related characteristics of stroke patients included in the analysis $(n=189)$

\begin{tabular}{ll}
\hline & $\mathbf{n}=\mathbf{1 8 9}$ \\
\hline Sex (female) & $47.1 \%$ \\
Age, yrs (mean, sd ; range) & $68.5(11.6 ; 36-89)$ \\
Level of education & \\
$\quad$ primary school & $42.5 \%$ \\
$\quad$ junior secondary vocational education & $25.5 \%$ \\
$\quad$ senior secondary vocational education & $19.0 \%$ \\
$\quad$ (pre-)university education & $13.0 \%$ \\
Living alone & $35.9 \%$ \\
MMSE (median, min-max; \% 523$)$ & $26(16-30 ; 21.5 \%)$ \\
FAST (median, min-max) & $27(9-30)$ \\
Barthel (mean, s.d.; median) & $17.5(4.6 ; 20)$ \\
Rankin (mean, s.d.; median) & $2.4(1.2 ; 2)$ \\
Level of Neuroticism (mean, s.d.) & $30.1(7.3)$ \\
Personal history of depression & $21.8 \%$ \\
Family history of psychiatric disorders & $36.0 \%$ \\
\hline
\end{tabular}

MMSE: Mini Mental State Examination (minimum score: 0, maximum score: 30); FAST: Frenchay Aphasia Screening Test (minimum score: 0, maximum score: 30); Barthel Index (completely dependent in Activities of Daily Living: 20, no disabilities: 0); Rankin (no handicaps: 0 , bedridden: 5)

stroke patients become handicapped are independent risk factors for depression after stroke. ${ }^{167}$

In this study, we studied the cumulative one-year incidence of PSD in 190 consecutive patients with a first-ever supratentorial infarct. Available CT or MR scans were used to evaluate focal lesion characteristics as well as the occurrence of generalised vascular damage. First, we tried to replicate the finding that left frontal strokes increase the risk for PSD. Secondly, we studied the occurrence of generalised vascular damage as a risk factor for PSD. Subsequently, using the same cohort, these two factors were combined into one multivariate analysis including other well-established risk factors of depression, such as female sex, history of depression, neuroticism and level of handicap.

\section{PATIENTS}

Between September 1, 1997 and September 1, 1999, 444 consecutive patients were diagnosed with a first-ever supratentorial brain infarct at the Emergency Department and the Outpatients Clinic of the University Hospital of Maastricht, 
the Netherlands. This University Hospital serves approximately 200,000 inhabitants and is the only hospital in the region.

Stroke was diagnosed by a neurologist according to the WHO criteria. ${ }^{149}$ Patients' data were entered into a prospective stroke registry (Maastricht Stroke Registry MSR), which has been described in detail elsewhere. ${ }^{127}$ The ischemic nature of stroke was verified by computed tomography (CT). Patients with other types of stroke (e.g. recurrent stroke, haemorrhage, or brain stem infarct) were not included in order to increase the homogeneity of the study groups.

Hundred-ninety-three (43.5\%) patients were excluded. Exclusion of patients who were unable to communicate reliably (e.g., because of severe aphasia or cognitive dysfunction) was based on combined clinical judgment and Mini Mental State Examination (MMSE) and Frenchay Aphasia Screening Test (FAST) results (see Methods section). Reasons for exclusion are shown in figure 1.

Sixty-one of the remaining 251 eligible stroke patients refused participation $(24.3 \%)$. Refusers were somewhat older than participants $(72.4 \pm 9.7$ vs. $68.6 \pm 11.7$ years; $\mathrm{t}(2)=2.6, \mathrm{p}=0.01)$. No sex difference was found between these two groups. Moreover, 35 refusers $(57.4 \%)$ who were willing to fill-out two psychiatric selfrating scales (SCL-90 and HADS, see below), did not report significantly more depressive symptoms than participants.

Thus, 190 stroke patients participated in the study. Major characteristics of this cohort are summarised in table 1. All participants gave written informed consent. The study was approved by the Medical Ethics Committee of the University Hospital Maastricht.

\section{METHODS}

\section{CT AND MRI SCANS}

All patients had CT in the acute phase of stroke. Of a subgroup of 75 patients, an additional MRI scan was made as part of a research project on stroke and reactive hypertension. ${ }^{168}$ All scans were judged by a neurologist (JL) who was blind to the clinical details of the stroke. Subsequently, discrepancies between CT or MR scorings and the clinical presentation of the stroke were detected and reconsidered. Data were collected on lesion characteristics and (pre-existing) vascular brain damage (see below) using all available data from the MSR, CT and MR scans. If available, MR data had priority over CT data. If no 'fresh' symptomatic infarct could be detected on any of the images, clinical data were used to determine type and side of the stroke lesion. ${ }^{169}$ This was the case in 51 of 113 patients who only had CT scans (45.1\%) compared to only 4 patients with an MR scan (5.3\%). The relatively high proportion of negative scans in patients with recent stroke is in line with other studies. ${ }^{170}$ In addition, the inter-rater agreement on the vascular imaging 
characteristics has been examined earlier by our group and was found to be excellent (kappa-scores $\geq 0.79)^{15}$

\section{LESION CHARACTERISTICS}

Stroke type, lesion location, and lesion size were determined. Initially, stroke types were divided into cortical, sub cortical, lacunar, centrum semi-oval or striatocapsular type. Because some of these types did not occur often, broad categories of territorial versus lacunar infarcts were made. Sub cortical and centrum semi-oval infarcts were considered territorial if their largest diameter exceeded 15 $\mathrm{mm}$. Infarcts including the cortex and striatocapsular infarcts were considered as territorial infarcts.

Figure 1. Patient selection and recruitment

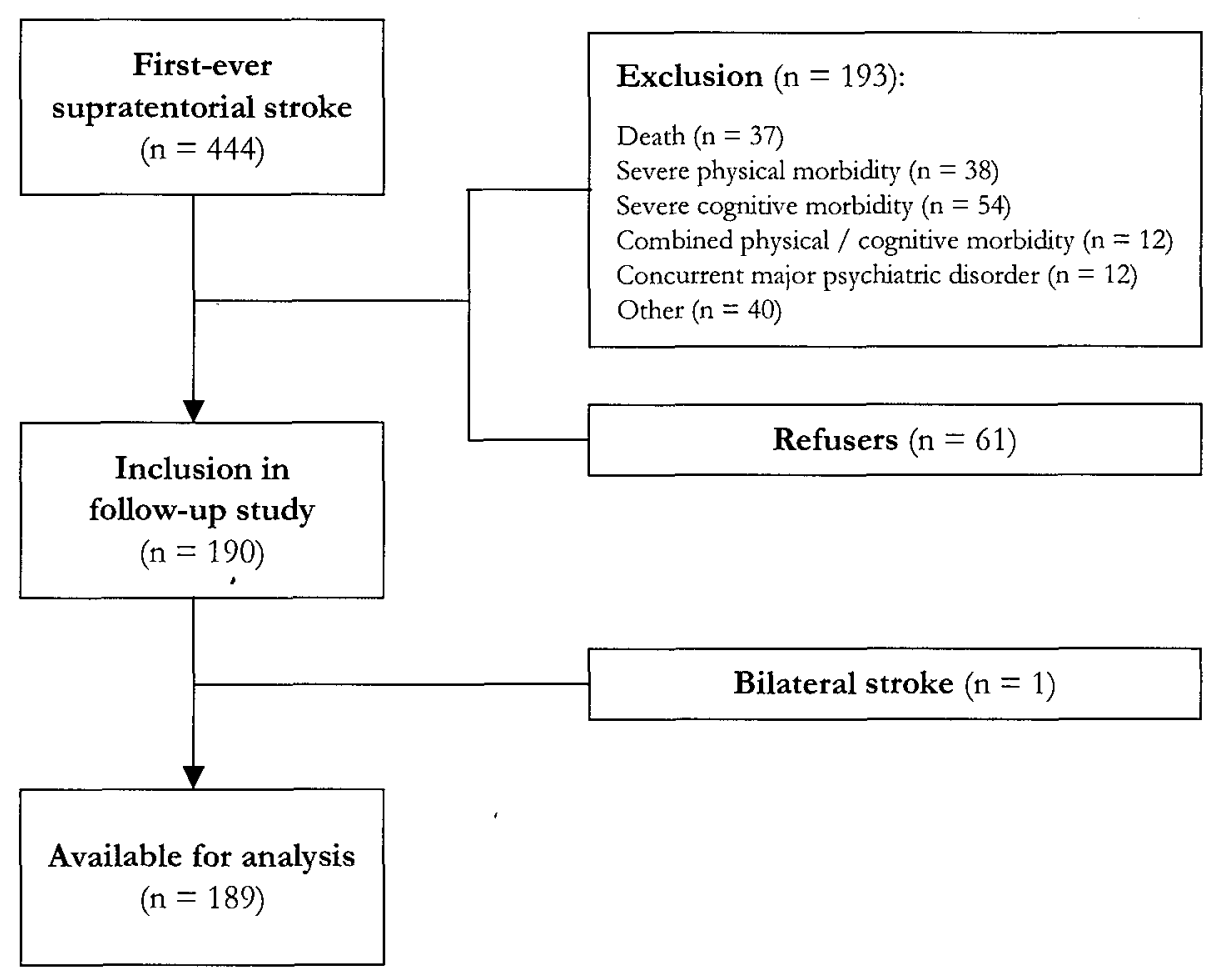


Lesion location was both expressed in terms of hemisphere involvement (left vs. right) and of involvement of the frontal region (further referred to as 'anterior' vs. 'posterior'). In the case of territorial infarcts with a positive scan, the central sulcus was taken as the divider between the anterior and the remainder part of the brain. Striatocapsular infarcts were considered to involve the frontal circuitry. In case of a negative scan, clinical stroke symptoms were used to decide about frontal involvement. Stroke syndromes restricted to visual field deficits, visuo-spatial deficits, agnosia, apraxia, or other type of "higher cortical dysfunction" were listed in the 'posterior' category, whereas those with frontal symptoms, such as Broca or mixed aphasia were considered as 'anterior'. In the case of lacunar infarcts, involvement of the frontal region was considered to be represented by damage to the head of the caudate nucleus, striate nucleus, and anterior leg of the internal capsule. If no infarct could be detected on CT or MRI scan, all regular lacunar syndromes were considered to have a posterior localisation because symptomatic lacunar infarcts are known to be mostly located in this region ${ }^{171}$ and it prevented us from falsely allocating infarcts without frontal involvement to the hypothesized high risk group.

In the case of a territorial infarct, size was rated on a semi-quantitative basis as small, medium or large. For reasons of statistical power, data were subsequently dichotomised into small vs. medium/large.

\section{GENERALISED VASCULAR BRAIN DAMAGE}

Leukoaraiosis can be defined as an abnormal appearance of the sub cortical white matter of the brain on neuro-imaging. On CT, it is characterised by bilateral patchy or diffuse areas of low attenuation, while on T2 MR it presents as hyper intense areas in the white matter. ${ }^{172}$ On MR scans, such vascular white matter lesions were scored using the Fazekas scale. This scale separately rates the different types of hyper intense signal abnormalities surrounding the ventricles and in deep white matter. Per ventricular hyper intensity (PVH) is graded as $0=$ absence, $1=$ 'caps' or pencil thin lining, $2=$ smooth 'halo', $3=$ irregular PVH extending into the deep white matter. Deep white matter hyper intense signals (DWMH) are rated as $0=$ absence, 1 = punctuate foci, 2 = beginning confluence of foci, 3 = large confluent areas. ${ }^{173}$

In case of CT data, leukoaraiosis was considered to be present if at least at one of both sides (periventricular or in deep white matter) patchy or diffuse areas of low attenuation were detected. Since CT scans are less sensitive to the detection of vascular white matter lesions and in order to enable pooling of CT and MR data, we recoded the Fazekas ratings into one overall dichotomous measure of leukoaraiosis (present vs. absent). Only in the case of confluent lesions, leukoaraiosis was rated as 'present'. 
Furthermore, asymptomatic (silent) infarcts were scored according to procedures described in more detail elsewhere. ${ }^{15}$ On MRI and CT, these are characterised by circumscriptive low-density areas (hyper dens in T2 MRI), compatible with infarction but without a history of any clinical signs or symptoms of stroke other than at study entry.

\section{INITIAL ASSESSMENT OF DEPRESSION}

All patients were followed up during the first year after stroke. PSD was defined as an episode of major or minor depression according to DSM-IV criteria ${ }^{47}$ on at least one assessment during the 1-year follow-up period. After one month, all patients were interviewed using both the depression section of the Structured Clinical Interview for DSM-IV (SCID-I-R) ${ }^{158}$ and the Hamilton Depression Rating Scale (HAM-D). ${ }^{96}$ The SCID is a structured psychiatric diagnostic interview allowing for a DSM-IV diagnosis of major or minor depression. The HAM-D is a clinical rating scale that measures the severity of depressive symptoms. All interviews were administered by the same clinician (IA), who was trained to use these instruments. No formal test of inter-rater reliability was performed.

\section{FOLLOW-UP ASSESSMENT OF DEPRESSION}

At 3, 6, 9, and 12 months after stroke, patients were asked to complete three psychiatric self-rating scales to screen for depression. These were the Beck Depression Inventory (BDI), ${ }^{69}$ the Hospital Anxiety and Depression Scale (HADS), ${ }^{129}$ and the 90-item Symptom Check List (SCL-90). ${ }^{136}$ Cut-off levels were $9 / 10$ for the BDI and $7 / 8$ for both the depression and the anxiety subscale of the HADS. In the case of the depression subscale of the SCL-90, the threshold was $22 / 23$ for men and $27 / 28$ for women. The predictive validity of these instruments in this cohort was previously analysed. ${ }^{151}$ The sensitivity of this screening procedure was shown to be $93.8 \%$ at the one-month assessment.

Patients whose scores exceeded the cut-off value for at least one of the self-rating scales were reinterviewed using the SCID and HAM-D in order to diagnose major: or minor depression. In $50 \%$ of these cases, the interview was within two weeks; in $75 \%$ within 3-3.5 weeks.

In 33 patients the follow-up assessment of depression was incomplete, while the event of interest (depression) had not yet occurred. Of these, 16 withdrew their consent, 5 died, 8 had too severe co-morbidity, 1 was lost to follow-up, and 3 did not respond at the final assessment (12 months). 


\section{Potential CONFOUNDERS}

Sex, age, personal of depression, family history of psychiatric disorders, level of handicap, and neuroticism were predefined as potential confounders or effect modifications in the hypothesised relation between focal or generalised vascular brain damage and PSD.

Data concerning demographics, level of education, living situation, and family history of psychiatric disorders were collected on inquiry 1 month after stroke. Level of disability and handicap were rated at the same time using the Barthel Index ${ }^{130}$ and Rankin score, ${ }^{131}$ respectively. Furthermore, personal history of depression was measured using the SCID-I-R, while the MMSE ${ }^{128}$ and FAST153 were administered to measure global cognitive functioning. Data on personal history of depression was missing in 4 cases, on family history of depression in 11 cases, and on Rankin score in 1 case.

Neuroticism was assessed one month after stroke, using the NEO Five Factor Inventory (NEO-FFI), ${ }^{156}$ which has been translated into Dutch. ${ }^{159}$ This self-report questionnaire consists of 60 statements covering the five main dimensions of personality: neuroticism, extraversion, openness to new experiences, agreeableness, and conscientiousness. Neuroticism has been related to depression most frequently $y^{160}$ and is defined as a stable disposition to experience psychological distress across time and situations consisting of negative emotions such as fear, anger, and frustration. ${ }^{156}$ Each statement is rated on a 5-point scale ranging from 'strongly disagree' to 'strongly agree', resulting in total dimension scores between 12 and 60 .

Non-response concerning the assessment of personality occurred in 36 patients $(18.9 \%)$, either because of study withdrawal or difficulty completing the NEO-FFI.

\section{ANALYSIS}

Since depressive outcome was measured prospectively on 5 different time points duting the 1-year follow-up, a survival analysis technique was applied by means of Cox regression. If data on depressive status on one assessment was missing, the patient was considered "not depressed" if the patient was not depressed at the former assessment and if the depressive status at the next assessment was validly measured. In all other situations, the case was excluded from further analysis from the time point of the next assessment onward.

Cox regression was used to analyse the relative hazard (HR) of the different measures of both focal and generalised vascular damage on the incidence of PSD (major and minor depression combined). First, hemisphere involvement (left vs. right) and orientation towards the frontal pole (frontal region included vs. frontal region not included) were analysed in an interaction model. Analogous, both 
measures of generalised vascular damage (leukoaraiosis and asymptomatic infarcts) were analysed in an interaction model.

Secondly, a multivariate model was tested including measures on both lesion location and generalised vascular damage, as well as potential confounders (sex, age, personal history of depression, family history of psychiatric disorders, level of neuroticism, and level of handicap -i.e. Rankin score).

One case had bilateral stroke and was excluded from further analysis. In order to optimise the statistical power, missing data on dichotomous variables were imputed with value 0 (=absent), while missing data on continuous variables were imputed with the mean value of that variable. Post-hoc analyses were carried out to explore the consequences of this technique on the results by using list wise deletion of cases with missing data on any of the variables in the equation. No interaction effects were hypothesised on an 'a priori' basis.

The output of the Cox analyses was checked for instability by influential cases and for violation of both the proportional hazards assumption and the assumption of linearity of effects. Where appropriate, Hazard ratios are given with their $95 \%$ confidence intervals and 2-tailed p-values as: HR $(95 \%-C I)$, p.

For group comparisons of descriptive sample characteristics, Student's t-test was used in the case of continuous normally distributed variables. The Chi-square test was used for all dichotomous variables. Finally, one-way ANOVA was used to compare differences in HAMD scores between major depressed, minor depressed, and non-depressed patients. The level of significance was set at $\mathrm{p}<0.05$ (2-tailed) for all analyses. Where appropriate, results are given as means \pm SD.

\section{RESULTS}

\section{ONE-YEAR CUMULATIVE INCIDENCE OF DEPRESSION}

The 1-year cumulative incidence of depression was 38.7\% (adjusted for cases with incomplete follow-up). Cross-sectionally, the incidence rates were $21.6 \%(41 / 190)$ at 1 month, $5.1 \%(7 / 137.5)$ at 3 months, $6.0 \%(7 / 117)$ at 6 months, $5.6 \%(6 / 107)$ at 9 months, and $7.1 \%(7 / 98)$ at 12 months. Of these, 41 patients $(23.3 \%)$ met DSM-IV criteria for major depressive disorder and $27(15.4 \%)$ met criteria for minor depressive disorder. The mean HAMD score was $19.2 \pm 4.1$ for the patients with major depression, $13.2 \pm 4.3$ for the patients with minor depression, and $7.3 \pm$ 4.1 for the non-depressed patients. These differences were statistically significant $(\mathrm{F}(2)=198.2 ; \mathrm{p}<0.001)$. 
FIguRE 2. Distribution of lesion characteristics (hemisphere and frontal region involvement)

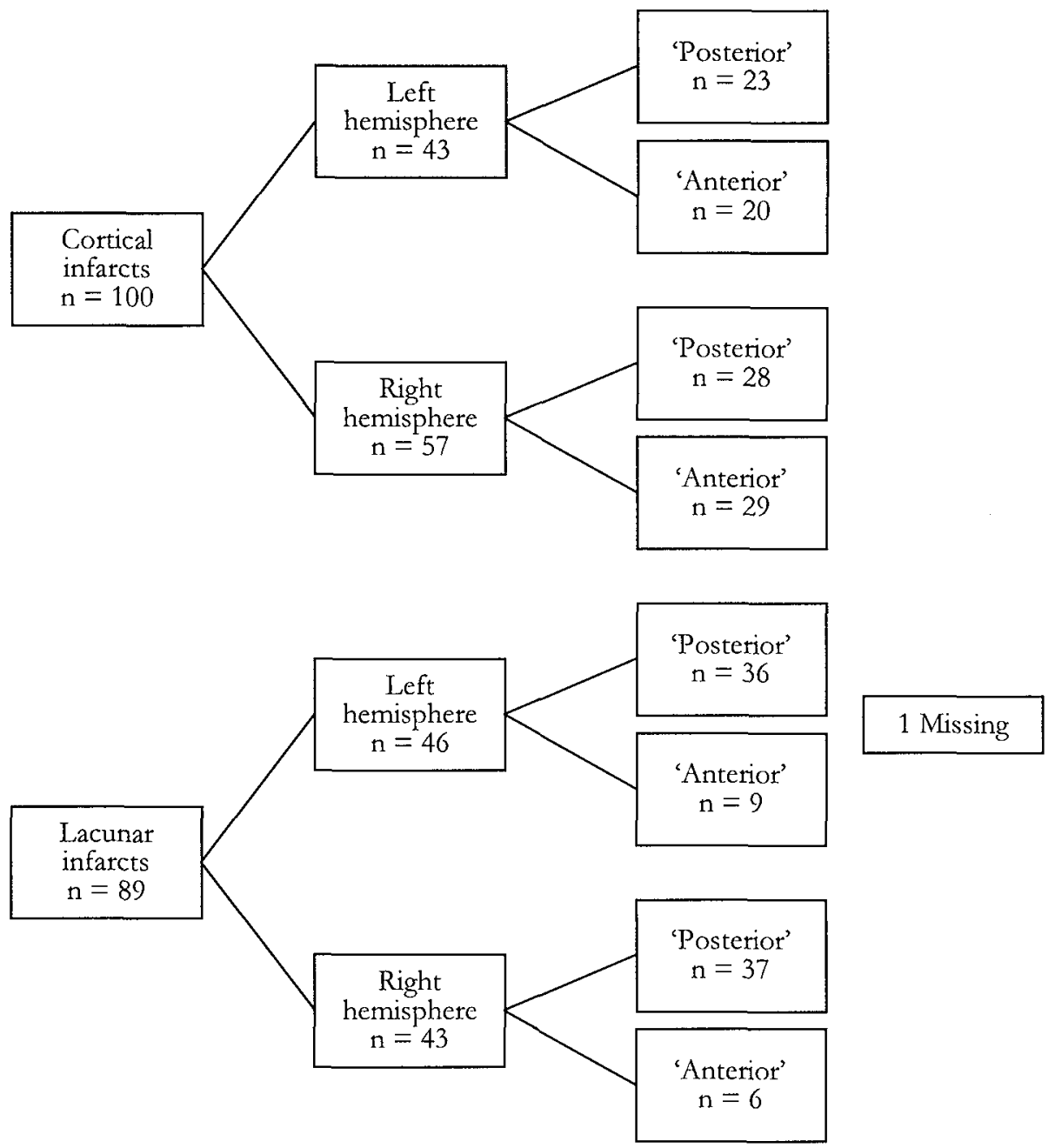

\section{DISTRIBUTION OF MEASURES OF FOCAL AND GENERALISED VASCULAR DAMAGE}

Of the 189 cases that were available for subsequent analysis on CT/MRI findings, $89(47.1 \%)$ had left sided strokes and in 100 patients $(52.9 \%)$ the stroke was due to a territorial infarct. In 64 strokes $(33.9 \%)$ the frontal region was involved. Figure 2 shows how these lesion characteristics were combined. Note the relatively low 'anterior' to 'posterior' ratio (15/73) in lacunar infarcts as compared to territorial 
infarcts (49/51). Size of infarct could only be rated in 75 of 100 cortical infarcts ( 25 werc not visible on scan). Seventeen of these were rated as small, 47 as moderate and 11 as large.

Eighty-six patients (45.5\%) showed one or more silent infarcts (i.e. not relating to clinical symptoms), while 59 patients had leukoaraiosis $(31.2 \%)$. Of these patients, 48 had a positive score on both of these measures of generalised vascular damage, 11 only had leukoaraiosis, and 38 only had one or more silent infarcts. Signs of generalised vascular damage occurred with equal frequency between territorial and lacunar infarcts: $49 / 100$ patients with a territorial infarct $(49.0 \%)$ showed any sign of generalised vascular damage compared to $48 / 89$ patients with a lacunar infarct $(53.9 \%)$.

\section{LESION CHARACTERISTICS AND GENERALISED VASCULAR DAMAGE: RELATION TO DEPRESSION}

In an attempt to replicate the finding that left sided strokes and/or strokes that involve the frontal region of the brain increase the risk of PSD, Cox regression was performed with side of lesion (left vs. right) and frontal region involvement ('anterior' vs. 'posterior') as independent variables. No significant interaction effect

TABLE 2. Multivariate Cox regression models for factors of both focal and gencralised vascular damage as risk factors for PSD after adjustment for potential other risk factors: all factors entered and forward stepwisc procedure $(n=189)$

\begin{tabular}{llll}
\hline Whole model & HR & $\mathbf{9 5 \% - C I}$ & P \\
\hline Territorial infarct (vs. lacunar) & 1.09 & $0.63-1.88$ & 0.77 \\
Left hemispherc & 1.11 & $0.06-1.86$ & 0.70 \\
Irontal region involved & 0.52 & $0.28-0.98$ & 0.04 \\
L.cucoaraiosis & 0.79 & $0.42-1.51$ & 0.48 \\
Asymptomatic infarct(s) & 1.28 & $0.73-2.24$ & 0.39 \\
Neuroticism (continuous scale) & 1.07 & $1.02-1.11$ & 0.003 \\
Handicap (ordinal scale) & 1.47 & $1.13-1.91$ & 0.004 \\
Family history of psychiatric dis. & 1.53 & $0.92-2.53$ & 0.10 \\
Personal history of depression & 1.30 & $0.71-2.35$ & 0.40 \\
Fenale sex & 1.28 & $0.75-2.18$ & 0.36 \\
Age & 0.99 & $0.97-1.02$ & 0.63 \\
\hline Stepwise model & $\mathbf{H R}$ & $95 \%-\mathrm{CI}$ & $\mathbf{P}$ \\
\hline Frontal region involved & 0.56 & $0.32-0.98$ & 0.04 \\
Neuroticism (continuous scale) & 1.08 & $1.04-1.12$ & $<0.001$ \\
Ilandicap (ordinal scale) & 1.43 & $1.14-1.79$ & 0.002 \\
lamily history of psychiatric disorders & 1.70 & $1.05-2.76$ & 0.03 \\
\hline
\end{tabular}

HR: Hazard Ratio. 
of these lesion characteristics was found (left*anterior: HR $0.60 \quad(0.20-1.79)$, $\mathrm{p}=0.36$ ). Nor was there evidence of a risk increasing effect of one of the separate variables in a subsequent confounding model (left: HR 0.92 (0.57-1.49), $\mathrm{p}=0.73$; anterior: HR $0.74(0.43-1.25), p=0.26)$. In addition, the effect of infarct size could only be tested in 75 patients with a territorial infarct. Bivariate Cox regression analysis revealed no such effect (HR $0.73(0.30-1.74), p=0.47)$.

Similarly, the attempt to replicate the finding that generalised vascular damage increases the risk of depression, failed to reveal an interaction effect for leukoaraiosis*asymptomatic infarcts: HR $1.93(0.40-9.38), \mathrm{p}=0.42$. A subsequent confounding model also failed to reveal independent effects of leukoaraiosis (HR $0.91(0.51-1.63), \mathrm{p}=0.75)$ or asymptomatic infarcts ( $\mathrm{HR} 1.30(0.76-2.23), \mathrm{p}=0.34)$ alone. Re-analysis of this model with one overall variable for generalised vascular damage confirmed the absence of a relationship with PSD.

In accordance with our suggestion that PSD should be considered as having a multifactorial path physiology, we subsequently tested one overall model including both the variables of focal and generalised vascular damage as well as non strokespecific risk factors sex, age, personal or family history of depression, neuroticism, and level of handicap.

Unexpectedly, in this multivariate model patients with 'posterior' infarcts had a significant higher risk of depression than patients with strokes that involved the frontal region (see Table 2). As expected from earlier analysis, ${ }^{167}$ from the variables that are not specifically stroke-related, neuroticism and level of handicap showed to be independent risk factors for PSD. In addition, a trend was shown for a positive family history of psychiatric disorders.

Post-hoc, forward stepwise regression analysis was performed to optimise the model resulting in a four-factor model including 'posterior' stroke, neuroticism, handicap, and family history of psychiatric disorders (Table 2).

Further post-hoc analyses were performed to test interaction effects between $\mathrm{CT} / \mathrm{MRI}$ variables and between $\mathrm{CT} / \mathrm{MRI}$ variables and non-stroke specific vulnerability factors (neuroticism, handicap and positive family history). No significant interaction effects came out of these analyses.

\section{DISCUSSION}

This study has attempted to examine the role of both focal and generalised vascular damage on the incidence of post-stroke depression in a single cohort of 190 firstever infarct patients who were followed for one year using standardised psychiatric diagnostic scales. Our findings neither support the lesion location hypothesis, nor the vascular depression hypothesis as explanatory models for PSD. 
As reported previously, ${ }^{167}$ our study does support, however, the proposition that non-stroke specific vulnerability factors contribute to the development of PSD. Both the level of neuroticism and handicap came out as independent risk factors, while a positive family history of psychiatric disorders seemed to further contribute to the risk of PSD. Therefore, in studying the role of biological (stroke-related) factors as independent predictors of PSD these determinants should also be considered. Although in our study we found no evidence of interaction between vulnerability factors and measures of focal or general vascular brain damage, we suggest that more powered studies could reveal such risk potentiating effects. For instance, vascular white matter lesions may contribute more to a person's vulnerability for depression when the patient has already suffered from depression in the past.

Much to our surprise, an unexpected protective effect of depression was found for infarctions with anterior involvement, when analysing the overall model with both stroke specific and non-specific factors. This finding opposes the proposed role of damage to the frontal region of the brain in inducing post-stroke or vascular depression and needs further elaboration. A possible explanation for this finding may be sought in the probable under-representation of left frontal strokes by the exclusion of patients with severe aphasia or other severe cognitive deficits from this study. As a consequence, other neurological deficits that are related to posterior brain dysfunction, such as visuo-spatial disturbance or hemi-neglect, may also trigger the development of PSD. As an alternative and more conservative explanation, the finding may be based on a type I statistical error. It must be noted that the significant effect was only found in multivariate (11 factor model and stepwise regression model) and not in bivariate analysis.

Since a variety of previous studies by others also failed to replicate the lesion location hypothesis, Robinson recently reported to have carried out a meta-analysis (in press) and claimed to have found an interaction between lesion location and time of onset of PSD. ${ }^{174}$ Left hemisphere lesions would increase the risk of PSD especially in the first few months after stroke, whereas in the chronic course psychosocial factors would become more important. We tried to test this modification of his original hypothesis by controlling for time-dependency in the Cox regression model but found no such effect. Interestingly, Gainotti had tested this same assumption already in 1999 and found that both the symptom profiles and anatomical-clinical correlates of major PSD were not different in the acute and more chronic stages of stroke. ${ }^{66}$

Despite of the negative findings that undermine the plausibility of the lesion location hypothesis, it cannot be rejected yet. A more precise delineation of specific brain structures that may be involved in mood regulation and, therefore, in the development of PSD, may result in more consistent evidence in favour of the lesion location hypothesis. Vataja et al. showed that this strategy seems hopeful, 
since in their study of 275 stroke patients, they found that infarcts affecting the prefrontosubcortical circuits, especially the caudate, pallidum, and genu of internal capsule (with left-sided predominance) were associated with a higher prevalence of PSD. 21

Concerning the vascular depression hypothesis, we argue that in a stroke population (pre-stroke) vascular brain damage as measured by leukoaraiosis and silent cerebral infarctions does not significantly contribute to the development of PSD, since the direct consequences of stroke itself (either biological, psychological or social) are so strongly depressogenic that the contribution of pre-existing small vascular lesions are overshadowed. In their study, Vataja et al. (2001) also failed to find an association between white matter lesions and PSD. This explanation is supported by the recent finding by Mast et al. (2004) that in a large cohort of 670 rehabilitation patients, depression was associated with increased burden of cerebrovascular risk factors in patients without stroke but not in stroke patients. ${ }^{175}$, 170 It is additionally noted that our group also failed to find a significant contribution of generalised vascular damage on the development of post-stroke cognitive disorders..$^{15}$

The main shortcoming of the study lies in the limited number of available MRI scans, so that we had to rely on CT data in a majority of cases. CT scans made in the acute phase of stroke failed to detect 'fresh' infarction of brain tissue in 53 of $114(46.5 \%)$ cases and they are also less sensitive in detecting vascular white matter lesions. Therefore, clinical data were used to complete data on lesion characteristics such as hemisphere involvement and type of stroke (territorial vs. lacunar). Additionally, in pooling CT and MR data, variables were dichotomised in order to reduce the chance of systematic measure errors.

Given the rather equal distribution of strokes over both hemispheres, it is not likely that exclusions have led to essential under-representation of right or left hemisphere strokes. Because of the relatively low frequency of infarcts with cortical involvement, of which a minority of $43 \%$ were located in the left hemisphere, it seems, however, that cortical strokes have been excluded relatively frequently, especially in the left hemisphere. One can imagine that patients with severe aphasia or generalised cognitive disabilities are especially vulnerable to depression, so that the cumulative incidence of PSD as reported in this thesis may be underestimated. These limitations may have contributed to the negative results of this study.

In conclusion, we found no support for both the lesion location hypothesis and the vascular depression hypothesis in stroke patients. In order to appreciate the lesion location hypothesis for its true value, future research should aim to overcome the methodological difficulties in PSD research such as the exclusion of patients with severe aphasia or other cognitive deficits in our study. Furthermore, a more detailed determination of neuronal circuits that are involved in mood regulation 
may also prevent the influential hypothesis of Robinson to be rejected on immature research findings. 


\section{CHAPTER 7}

Risk factors for post-stroke depression: a two-step Cox regression analysis

This chapter is submitted for publication as.

Leentjens A, Aben I, Lodder J. Verhey F. Risk factors for post-stroke depression: a two-step Cox regression analysis (submitted) 


\section{ABSTRACT}

Background: Post-stroke depression commonly complicates stroke and is associated with an impaired functional outcome, more severe cognitive deficits, reduced quality of life, and higher mortality.

Objective: To assess whether general risk factors for major depressive disorder are also risk factors for post-stroke depression, and to identify additional, strokerelated, risk factors.

Patients and methods: One hundred-and-ninety consecutively admitted patients were assessed for major depressive disorder one month after stroke, and after 3,6 , 9 and 12 months. A two-step Cox regression analysis was performed. A first model was created of five established risk factors for major depressive disorder in the community (older age, female sex, a positive personal and family history of depression, and somatic comorbidity other than stroke). Next, five potential disease-related risk factors (disability, cognitive deterioration, inter- and intrahemispheric lesion location, and generalized vascular damage on CT scan) were added to this model one by one, to see whether these would improve the significance of the overall model.

Results: The Cox model of five general risk factors for depression in the community showed itself to be a valid model for predicting depression in stroke patients. 'Disability' was the only disease-related variable that improved the model. Though related to stroke, it cannot be considered disease-specific, as disability is a non-specific sequel of many diseases.

Conclusion: Established risk factors in the community are also predictors of depression in stroke patients. Disability is a non-specific disease-related variable that is associated with post-stroke depression. The contribution of specific strokerelated factors may be less than generally expected. 


\section{INTRODUCTION}

Major depression is one of the most common psychiatric disorders, with a reported prevalence of $1.8 \%$ (range 0.4 to $35 \%$ ) in the community. ${ }^{177}$ In patients with stroke, the prevalence of so-called post-stroke depression (PSD) is substantially higher. Reported prevalence rates of post-stroke major depression range from 13\% in community studies to $23 \%$ in neurological in- and outpatient populations. In addition, minor depression is present in $10 \%$ of stroke patients in the community, in $35 \%$ of patients visiting the neurological outpatient department, and in $17 \%$ of neurological inpatients. ${ }^{6}$ In stroke, major depression has been associated with impaired functional outcome, more severe cognitive deficits, reduced quality of life, and a higher mortality. ${ }^{28,65,178,170}$ Prevention of PSD may therefore improve the prognosis of stroke patients, as well as reduce the costs of medical utilization and care. Knowledge of risk factors for depression after stroke may make it possible to enable the identification of patients at risk of depression and, thus, facilitate targeted interventions. These interventions may be both primary prevention of post-stroke depressive disorder, and secondary prevention, i.e. early recognition and treatment.

Several large scale prospective epidemiological studies have identified risk factors for depression in the general population, such as female sex, older age, being single, lower level of education, the presence of physical disease, and a personal history of depression. ${ }^{180-183}$ Other factors are less firmly established as a risk for depression, such as cognitive impairment, a family history of depression, smoking and alcohol consumption. ${ }^{184,} 185$ It may be assumed that these general risk factors for depression in the community also constitute risk factors for depression in stroke patients, although no study has yet specifically addressed this issue. If this is indeed the case, a multivariate statistical approach should control for the influence of these general risk factors when searching for specific stroke-related risk factors for depression. Moreover, this is necessary because it has become increasingly clear that depression is the result of multiple interacting risk factors. In stroke patients, previous depression, female sex, being single, and higher neuroticism scores, may be aspecific factors associated with a risk of depression.77, 10., 167, 186 A number of prospective studies have addressed potential specifically stroke-related risk factors for depression. Some have found that increased functional impairment, more severe cognitive impairment, previous stroke, left-sided lesion location, and involvement of anterior regions of the brain constitute risk factors for PSD, 75, 1013, 164, 186-188 although this has not been confirmed in other studies.22, 23, 10.3, 189 However, only a few studies have followed a multivariate approach and corrected for some of the established general risk factors for depression in the community. The aim of this study is to evaluate whether risk factors for depression in the general population are also risk factors for PSD, and to assess whether there are 
additional, stroke-specific risk factors, after correction for the influence of these general risk-factors for depression.

\section{PATIENTS}

From September 1, 1997 and September 1, 1999, 190 consecutive patients with a first-ever cerebral infarction underwent a standardised assessment. These patients were recruited from both the Accident \& Emergency Department and the Department of Neurology of Maastricht University Hospital. The diagnosis of stroke was made by a neurologist on the basis of the World Health Organisation (WHO) criteria. ${ }^{149}$ The ischemic nature of the stroke was verified by a computed tomography (CT-) scan. In the absence of ischemic changes on the CT scan, differentiation of left-sided and right-sided stroke was made on the basis of the clinical presentation. In order to obtain a homogenous population, patients with other types of stroke, such as haemorrhage, recurrent stroke, or brain stem infarction, were excluded from the study. More details on selection criteria of the study-population are described in an earlier publication. ${ }^{167}$

\section{METHODS}

All patients were followed during the first year after stroke. A first assessment took place one month after stroke. All patients underwent the Structured Clinical Interview for DSM-IV Depression (SCID-D) in order to confirm or reject a diagnosis of 'major depressive disorder' according to the criteria of the fourth edition of the Diagnostic and Statistical Manual (DSM IV) of the American Psychiatric Association (APA). ${ }^{47}, 158$ The severity of depressive symptoms was assessed with the Hamilton Rating Scale for Depression. ${ }^{60}$ The cognitive status of patients was assessed with the Mini-Mental State Examination (MMSE). ${ }^{128}$ Patients were asked to complete three self-rating scales for depression. These included the Beck Depression Inventory (BDI), the Hospital Anxiety and Depression Scale (HADS) and the Symptom Checklist-90 (SCL-90), which includes a 'depression' subscale. ${ }^{69,} 129,136$

Information on potential risk factors was gathered during a clinical interview. We focused on five established risk factors for depressive disorder in the general population and five potential disease-related risk factors. The general risk factors included: age, sex, prior personal history of depression, positive family history of depression in first-degree or second-degree relatives, and the presence of comorbid somatic disorders other than stroke, resulting in actual disability or handicap or need for medical attention. The potential disease-related risk factors for depression 
were the level of handicap, cognitive impairment, the side of stroke, the involvement of frontal brain regions, and the presence of generalized vascular damage on CT scan.

In order to classify a stroke as involving the frontal region versus the remaining parts of the brain, the central sulcus was chosen as the dividing structure. Striatocapsular infarcts were considered to involve the frontal circuitry. In case of a negative scan, clinical symptoms were used to classify the anterior-posterior location. Stroke syndromes restricted to visual field deficits, visuo-spatial deficits, agnosia, apraxia, or other higher cortical dysfunction were listed in the 'posterior' category, whereas those with frontal symptoms, such as expressive or mixed type aphasia were listed in the anterior category. Vascular damage was quantified by a neurologist on the basis of a modified Fazekas score, based on the presence of periventricular white matter hyperintensities, deep white matter hyperintensities, and subcortical grey matter lesions. Disability was rated with the Rankin score. ${ }^{131}$ All interviews were performed by the second author.

Follow-up took place after 3, 6, 9, and 12 months. Patients were screened for depression with the same three self-rating scales. If, on one of these scales, they scored above the cut-off point for 'depression', they were called in and interviewed. Arbitrary cut-off points for depression were set at $9 / 10$ for the BDI (meaning that 9 or lower is not considered indicative for depression, and 10 or higher is suspect for depression), and 7/8 for the HADS. On the depression subscale of the SCL-90, the cut-off was set at $22 / 23$ for men, and $27 / 28$ for women. ${ }^{190}$ Confirmation of the diagnosis of 'major' depression was done with the SCID-D interview.

\section{STATISTICS}

Two consecutive Cox regression analyses were performed with 'major depressive disorder' as outcome variable and the potential tisk factors as independent predictive variables. In the first analysis a multivariate model was constructed with the five established risk factors for depression in the general population. Hazard ratios (HR) with their concomitant $95 \%$ Confidence Interval (95\%-CI) and p-value were calculated for each factor from both bivariate and multivariate analyses. In the second part of the analysis, the five potential disease-specific risk factors were separately added to this model of general risk factors for depression, in order to see if they independently increase the risk for PSD and if they improve the strength of the overall model. The level of significance was arbitrarily set at 0.05 (two tailed). All analyses were performed with the Statistical Package for the Social Sciences (SPSS), version 10.0 (SPSS Inc. Chicago, 1998). 
TABle 1. Patient characteristics of the 167 patients included and the 25 patients excluded from the analysis. The significance $(\mathrm{p})$ of the between-group test statistic ( $t$ or Chi-square) is also tabulated. ( $\mathrm{SD}=$ standard deviation).

\begin{tabular}{llll}
\hline Characteristic & $\begin{array}{l}\text { Included patients } \\
\mathbf{n}=\mathbf{1 6 5}\end{array}$ & $\begin{array}{l}\text { Excluded patients } \\
\mathbf{n = 2 5}\end{array}$ & $\mathbf{p}$ \\
\hline Sex (\% female) & 46 & 52 & 0.58 \\
Average age (SD) & $68.1(11.8)$ & $71.4(10.5)$ & 0.19 \\
Somatic comorbidity (\%) & 86 & 92 & 0.47 \\
Personal history of depression (\%) & 21 & 29 & 0.40 \\
Family history of depression (\%) & 53 & 43 & 0.57 \\
Average Rankin score (SD) & $2.3(1.2)$ & $2.9(0.9)$ & 0.01 \\
Average MMSE score (SD) & $26(3.0)$ & $26(3.0)$ & 0.84 \\
Side of lesion (\% left-sided lesion) & 47 & 46 & 0.9 \\
Involvement of anterior regions & 34 & 33 & 0.95 \\
Vascular damage (\% evidence on CT scan) & 52 & 57 & 0.65 \\
\hline
\end{tabular}

\section{RESULTS}

Because Cox regression does not allow subjects with missing data on any of the independent variables to participate in the analysis, only 165 of the 190 screened patients could be included in the analysis. Twenty-five patients were excluded because of missing data on one or more of the risk factors, mainly on current somatic comorbidity and on family history of depression. The excluded group was no different from the included group with respect to age, sex, and other studied parameters, with the exception of a slightly higher Rankin score. The remaining patients were 89 men and 76 women, with an average age of 68.1 years (SD 11.8). Thirty-four patients (21\%) had a previous history of depression, while $58(35 \%)$ had a positive family history of depression. Somatic comorbidity was present in 140 patients $(86 \%)$. Most patients were moderately disabled, with an average Rankin score of 2.3 (SD 1.2). The average MMSE score was 25.9 (SD 3.0). In 87 patients $(53 \%)$ the lesion was in the right cerebral hemisphere, and in $85(52 \%)$ there was evidence of generalized vascular damage on the C'T-scan. In fifty-six patients $(34 \%)$, stroke involved the anterior regions of the brain. During the follow-up period, 38 patients developed major depression. The one-year cumulative incidence of major depressive disorder was $23 \%$. An overview of patient characteristics is given in table 1 .

In the first stage of the regression analysis, a Cox-model was created that incorporated the five risk factors for depression in the general population. The bivariate significance of these risk factors are shown in table $2 \mathrm{a}$, while the significance of the contribution of these risk factors in a multivariate Cox-model 
TABLE 2 (A and B). Hazard ratios (HR), 95\% confidence intervals (CI), and two-tailed $\mathrm{p}$-values of the five general and five disease-specific risk factors for depression in stroke are tabulated, in a bivariate, as well as in a five-factor Cox regression model. The significance of the multivariate Cox-model was $0.01\left(\chi^{2}=14.9, \mathrm{df}=5\right)$.

A. Bivariate model

\begin{tabular}{llll}
\hline Risk-factor & HR & 95\%-CI & 2-tailed p \\
\hline Female sex & 1.80 & $0.93-3.47$ & 0.08 \\
Age & 1.01 & $0.98-1.04$ & 0.56 \\
Somatic comorbidity & 2.03 & $0.62-6.57$ & 0.24 \\
Personal history of depression & 1.98 & $0.99-3.94$ & 0.05 \\
Family history of depression & 2.51 & $1.31-4.82$ & 0.006 \\
\hline
\end{tabular}

B. Multivariate Cox-model

\begin{tabular}{llll}
\hline Risk-factor & HR & $\mathbf{9 5 \% - C I}$ & 2-Tailed p \\
\hline Female sex & 1.54 & $0.78-3.04$ & 0.21 \\
Age & 1.01 & $0.98-1.04$ & 0.41 \\
Somatic comorbidity & 1.55 & $0.47-5.16$ & 0.48 \\
Personal history of depression & 1.65 & $0.80-3.40$ & 0.17 \\
Family history of depression & 2.20 & $1.11-4.33$ & 0.02 \\
\hline
\end{tabular}

are shown in table $2 \mathrm{~b}$. Although a family history of depression was the only variable that in itself contributed significantly to the multivariate model, the model as a whole was significant $(\chi 2=14.9, \mathrm{df}=5, \mathrm{p}=0.01)$. In the bivariate approach a previous history of depression and a family history of depression were significant predictors of depression ( $p=0.05$ and 0.006 , respectively), while there was a trend for sex as a predictor $(p=0.08)$. These outcomes clearly showed that there were confounding effects between the different variables: in the bivariate analyses all factors revealed, more or less, higher hazard ratios and were more significant than in the multivariate model (with the exception of age), showing that the associations between the different factors and PSD were not independent of each other.

In the second step of the analysis, potential disease-specific risk factors for depression were individually added to the Cox-model, in order to see whether this would lead to improvement of the model. Table 3 shows the odds ratios and significance of the resulting six-factor models after inclusion of each of the potential disease-related risk factors. Although the resulting six-factor models were all significant, only the model that incorporates the Rankin score as a predictor, significantly improved the significance of the model incorporating the five general risk factors for depression $(p=0.003)$. 
TABLE 3. Hazard ratios (HR), 95\% confidence intervals (CI), and two-tailed p-values of five general risk factors for depression and four disease-specific factors as risk factors for depression in stroke patients. The four disease-specific factors were separately added to the general model. The two-sided p-values of the resulting six-factor models are shown as well.

\begin{tabular}{lllll}
\hline Risk factor & HR & 95\%-CI & 2-Tailed p & Six-factor model p \\
\hline Rankin score & 1.53 & $1.09-2.14$ & 0.01 & 0.003 \\
MMSE score & 0.97 & $0.87-1.09$ & 0.64 & 0.02 \\
Left hemisphere lesion & 0.86 & $0.46-1.65$ & 0.64 & 0.02 \\
Frontal involvement & 0.94 & $0.46-1.90$ & 0.85 & 0.02 \\
Vascular damage on CT scan & 1.44 & $0.72-2.85$ & 0.30 & 0.01 \\
\hline
\end{tabular}

\section{DISCUSSION}

In this analysis, we assessed whether risk factors for depression in the general population are also risk factors for post-stroke depression, and whether there are additional, disease-related risk-factors, in stroke patients. Because of the sample size limitations that Cox regression sets, the number of potential risk factors included in the analysis was ten. Thus, we were unable to look at various other potential risk factors for depression, such as neuroticism, marital status, smoking, the use of alcohol, and the level of improvement of disability over time. The percentage of patients that developed major depression during the follow-up period is comparable to prevalence rates mentioned in review articles. ${ }^{6,161}$

The five-factor model of established risk factors for depression in the community (including age, sex, comorbid somatic disorders, a prior history of depression, and a positive family history of depression) was shown to be a valid model to explain a number of depressive outcomes in stroke patients. From the differences in the effect size of several factors between the bivariate and multivariate analyses, it can be inferred that the bivariate effects are confounded. In the multivariate model, a positive family history was the only factor that significantly increased the risk for PSD independently from the other factors.

The literature on age and sex as potential risk factors for PSD is inconsistent, and mostly based on studies that follow a (multiple) bivariate approach. Some studies found female sex as a risk factor for PSD, ${ }^{77,} 103,191$ while others did not. ${ }^{75,103,189}$ In a community study on risk factors for depression (not specifically addressing PSD), Sonnenberg et al. showed that the preponderance of depression in women in the community might be due to a greater exposure of competing risk factors. Adjusting for age, comorbid somatic disease, and a number of other risk factors reduced the 
relative risk of depression for women by more than half. ${ }^{192}$ Concerning age, some researchers have found that older age is associated with an increased risk of PSD, ${ }^{193}$ while others have found that younger age is associated with a higher risk of PSD. ${ }^{50}$, ${ }^{103}$ Some studies found more complex relationships. ${ }^{194}$ However, most studies did not find a relationship at all. 186,195 This is in line with our analysis, in which age is the factor that contributes the least in both bivariate and multivariate analysis.

A previous personal history of depression has been shown to constitute a risk factor for PSD in one study. ${ }^{186}$ Although we confirmed this finding in a bivariate Cox regression model, the effect disappeared after controlling for the other general risk factors. To the authors' knowledge no study has yet looked at a positive family history of depression in stroke patients, while in this analysis it was just this factor that came out as the most significant general risk factor for PSD.

Potential disease-related factors, such as cognitive impairment, lesion location, and leukoaraiosis on CT, could not improve the five-factor model based on general risk factors. As already mentioned, neurocognitive deficits is no well-established risk factor for PSD. Some studies showed a relationship, but these were mostly based on a bivariate approach and cross-sectional design. ${ }^{65,} 75$ Others reported negative findings. ${ }^{103,} 196$ If cognitive impairment in stroke patients is indeed a risk factor for PSD, it can only be addressed in prospective studies, because of the fact that depression itself may cause neurocognitive deficits.

Two recent systematic reviews did not support left-sided lesion location as a riskfactor for PSD. 22,23

In our analysis, the only disease-specific factor that improved the strength of the general model was the Rankin score, as a measure of disability and handicap. Several studies confirm an association between disability and PSD,6, 75, 103 The question is whether disability can be seen as a specific stroke-related factor. Disability is known to be a risk factor for depression in the community, as well as in patients with a variety of diseases such as cardiovascular disease, diabetes, cancer, and neurodegenerative diseases such as Alzheimer's disease or Parkinson's disease. ${ }^{197-199}$

Specific disease-related factors, especially factors relating to the pathophysiology and location of stroke, lack to contribute significantly to a multivariate risk factor model of PSD. To some extent, this is comparable to findings in other neuropsychiatric populations, such as in Parkinson's disease (PD) patients. Using a similar approach, our group has shown that potential disease-specific risk factors for depression in PD did not improve a general risk factor model of depression. In this study, only the side of onset of Parkinson's disease symptoms showed a weak association with depression. ${ }^{200}$ In Alzheimer's' disease, a similar two-step logistic regression approach failed to identify disease-related factors as risk factors for depression [Van Winkel R, Leentjens AFG, Lousberg R, Verhey FRJ, submitted]. 
There may be several explanations for this. Initially we may have been looking at the wrong disease-related factors. However, most clinically relevant factors have been repeatedly looked at in different studies. A second explanation, that appears to be more likely, may be that we should focus less on risk factors that are specific to one particular disease, but more to factors that many diseases have in common. This is not commonly done in research of depression in physical illness. Physical illness, as well as psychological stress, activates stress responses in an attempt to maintain homeostasis. Alterations in physiological mediators, such as adrenalin, glucocorticoids, and cytokines, alterations in the activity of the hypothalamichypopituitary-adrenal axis, and changes in neurotransmitter activities may induce depression, and even, if present for a prolonged time, lead to allostatic load. ${ }^{201}$ Stroke, other physical and neurological diseases, as well as disability, may compromise these adaptive responses, and constitute a non-specific disease-related risk of depression.

\section{CONCLUSION}

A Cox regression model based on five risk factors in the general population (age, sex, comorbid somatic disease, a past history of depression, and a positive family history of depression) showed itself to be a valid model to explain a number of cases of depression in stroke parients. Level of disability was the only diseaserelated factor that further improved this model. Cognitive symptoms associated with stroke, lesion location and evidence of leukoaraiosis did not improve the model. Based on the aspecificity of the effect of disability on PSD, and based on similar findings in other neurological and non-neurological diseases, it is suggested that aspecific factors related to disease in general may offer a more likely explanation for the high prevalence of PSD, than factors that are specifically related to the pathophysiology of stroke. In recognition of the complex nature of depression, future research should follow a multivariate approach that corrects for general risk factors for depression in the community. Moreover, it would be worthwhile to include parameters of physiological stress in such a multivariate model, which may reflect the final common pathway in the pathophysiology of depression in the course of any somatic disease. 


\section{CHAPTER 8}

Recognition and treatment of post-stroke depression in general practise

This chapter was published in Dutch as:

Aben I, Verhey F, Beusmans G, and Lodder J. Depressie na een beroerte: signalering, diagnostiek en behandeling in de huisartsenpraktijk. Huisarts en Wetenschap (2003), 46;487-492 


\section{ABSTRACT}

Goal: to determine how often depression after stroke is recognised and treated by general practitioners.

Methods: as part of a one-year prospective follow-up study of post-stroke depression, in 190 patients with a first-ever supratentorial infarct depression was diagnosed according to the DSM-IV criteria using a standardised diagnostic interview (SCID-I). General practitioners (GPs) of 58 of the patients who where discharged from hospital were interviewed about patient-contacts during the first year after stroke,

Results: depressed patients visited their GPs as often as non-depressed patients, but they reported more mental complaints. Depression as diagnosed with the SCID-I was recognised as such by the GPs in 11 of 29 cases $(37.9 \%)$. After diagnosing, an anti-depressive drug was prescribed in $50 \%$ of the cases whereas in the other $50 \%$ of the cases a follow-up appointment was made. The severity of depression and the nature of the complaints mentioned during history taking (somatic/mental) were significantly associated with adequate recognition of poststroke depression. Patient-related factors such as age, sex, level of education, personality traits, and level of handicap were not found to be of significant influence.

Conclusion: In about 50\% of the cases post-stroke depression is recognised by GPs. Anti-depressive drugs are prescribed in about half of these cases, as is the arrangement of a follow-up appointment. Recognition of post-stroke depression in general practise could be improved. Suggestions are made how to achieve this. 


\section{INTRODUCTION}

The majority of stroke patients is, eventually after a period of clinical rehabilitation, discharged to their homes. Therefore, general practitioners can play an important role recognising psychiatric disorders that occur after stroke. Depression is the most frequent psychiatric disorder after stroke.6, 20, 202 Recognising and treating depression in patients after stroke is essential for the well-being of these patients. This is not only because depression causes personal suffering but it also negatively affects the recovery from stroke. The stay of depressed stroke patients in a hospital is longer; they profit less from rehabilitation programs and have higher risks of dying within one to ten years after stroke. $27,28,31,106$ Treating post-stroke depression by using antidepressants showed to be effective in various studies. ${ }^{203,} 204$ The right guidance and treatment of depressed patients could also have a positive effect on revalidation and resocialisation.

In recent literature there is discussion on how often depression in elderly is recognised and treated by GPs. Several studies showed low percentages of about $50 \%,{ }^{205-210}$ while others reported more favourable results. ${ }^{211}$, 212 Adequate recognition has been shown to depend on several factors. Patient-related factors, such as male sex, higher age, somatic co-morbidity, handicap and coping styles (normalising attributions, denial), depression-related factors such as mild severity, shorter duration and symptom profile (more somatic complaints), and GP-related factors such as limited knowledge and skills, lack of time and a large patient load, all complicate adequate recognition of depression in the elderly.213-217 After stroke several specific problems are added to this list, such as physical handicap, and cognitive and phasic disturbances.

The recognition of post-stroke depression was only specifically studied in a few studies. In the Oxford Community Stroke Project, notes about psychological problems during the first year after stroke were only found in the records of 17 out of 128 patients $(13 \%)$. However, it could not be traced if these patients already suffered from depression at that time. ${ }^{80}$ In a recent Dutch study (Loor et al, 1999), 9 out of 71 stroke patients (13\%) exceeded the threshold of a depression questionnaire (Zung Depression Scale, shortened Dutch version) 2 months after stroke, while their GPs found 14 of 71 patients suffering from depression at any time between 8 and 12 months after stroke using a 4-monthly rated questionnaire concerning co-morbidity and functional status. It was concluded by the authors that the concordance between the Zung score and the judgement of the GPs were satisfactory (Cohen's kappa 0.64). However, some critical remarks can be made here. It cannot be ruled out that GPs were more aware of depressive symptoms in their patients after repeatedly filling out the questionnaire. Moreover, both the Zung scores and the judgement of the GPs cannot be equalled to a depressive disorder according to DSM-IV criteria. 
TABLE 1. Demographic and outcome-related characteristics of stroke patients of whom the GP was $(n=58)$ and was not $(n=71)$ interviewed

\begin{tabular}{|c|c|c|c|}
\hline & \multicolumn{3}{|c|}{ GP interviewed } \\
\hline & $\begin{array}{l}\text { Yes } \\
\mathrm{n}=58\end{array}$ & $\begin{array}{l}\text { No } \\
\mathbf{n}=71\end{array}$ & $\mathrm{P}$ \\
\hline Sex (female) & $44,8 \%$ & $43,7 \%$ & 0,89 \\
\hline Age, years (mean, SD) & $68,2(10,8)$ & $65,9(12,4)$ & 0,28 \\
\hline $\begin{array}{l}\text { Level of education } \\
\text { primary school } \\
\text { junior secondary vocational education } \\
\text { senior secondary vocational education } \\
\text { (pre-)university education }\end{array}$ & $\begin{array}{l}41,4 \% \\
22,4 \% \\
24,2 \% \\
12,1 \%\end{array}$ & $\begin{array}{l}38,0 \% \\
25,4 \% \\
19,7 \% \\
16,9 \%\end{array}$ & 0,21 \\
\hline Living alone & $35,7 \%$ & $27,1 \%$ & 0,30 \\
\hline MMSE (median, min-max) & $26(19-30)$ & $27(21-30)$ & 0,04 \\
\hline FAST (median, min-max) & $27(15-30)$ & $27(15-30)$ & 0,33 \\
\hline Rankin (mean, s.d.) & $2,4(1,1)$ & $1,8(0,9)$ & 0,001 \\
\hline
\end{tabular}

MMSE: Mini Mental State Examination (minimum score 0; maximum score 30); FAST: Frenchay Aphasia Screening Test (minimum score 0; maximum score 30); Rankin (no handicaps 0; bedridden 5).

The goal of the presented study was to gain more insight into the recognition and treatment of depression after stroke in (Dutch) general practises. Therefore, in a prospective study on the incidence and risk factors of post-stroke depression, inventory was made of the extent to which GPs were aware of depressive problems in their patients. A standardised telephone interview was developed for this purpose.

\section{PATIENTS}

As part of a prospective study on the incidence and risk factors of post-stroke depression, 190 patients diagnosed with a first-ever supratentorial infarct at the emergency department of the University Hospital Maastricht were followed up for 1 year. A detailed description of the design and methods of this project and of the patient characteristics has been published elsewhere. ${ }^{167}$

Patients with either co-morbid intracerebral disease or severe psychiatric disorder (including depressive disorder) that already existed prior to stroke, were excluded from participation, as were patients who were unable to be interviewed reliably due to their physical or mental (cognitive) condition. The study was approved by the Medical Ethical Committee of the hospital. All patients gave written informed consent. 
FIGURE 1. Flow chart

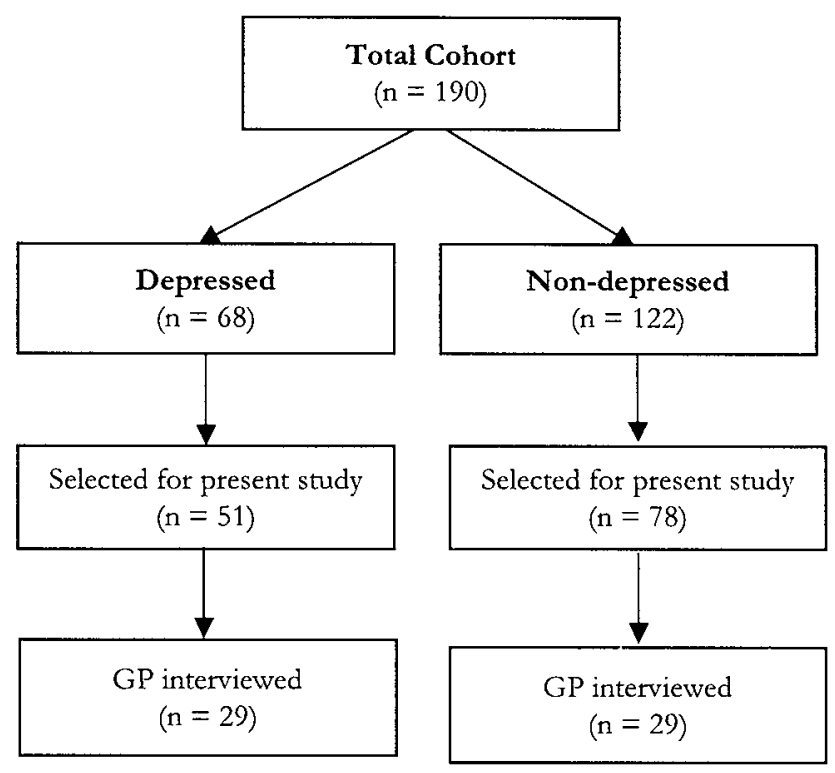

For the purpose of the present substudy a selection was made of patients who were, eventually after a period of clinical rehabilitation, discharged from the hospital to their homes. Patients who were admitted to a rehabilitation clinic or nursery home were excluded. Furthermore, patients who were found to be suffering from post-stroke depression were only selected if the diagnosis was made during the period after discharge from hospital, while patients who were never found to suffer from post-stroke depression were only selected if valid data were available from all assessments during the one-year follow-up period.

In total, 129 patients met these criteria (Figure 1). Of those 129 patients 51 were diagnosed with post-stroke depression at any time during the follow-up period. Of these 51 patients the GPs of 29 were interviewed. GPs of 9 patients refused cooperation, while the GPs of 13 patients were not available for the interview repeatedly (10 different GPs in total; due to death, illness, retirement, being abroad, or not being available to the researcher repeatedly). As a control group the GPs of 29 (out of 78) non-depressed patients were also interviewed. This subgroup was selected at random. There were 58 interviews in total with 39 different GPs.

Patients of whom the GP was interviewed and patients of whom the GP was not interviewed did not differ concerning age, sex, level of education, and civil status. However, patients of whom the GP was interviewed were more handicapped 
TABLE 2. Diagnostic symptom criteria for depression according to DSM-IV

\begin{tabular}{|c|c|}
\hline Core symptoms & $\begin{array}{l}\text { At least one of the following symptoms must be present most of the day, } \\
\text { nearly every day, for at least } 2 \text { weeks: } \\
\text { - Depressed mood } \\
\text { - Anhedonia (loss of interest and the ability to enjoy) }\end{array}$ \\
\hline $\begin{array}{l}\text { Additional } \\
\text { symptoms }\end{array}$ & $\begin{array}{l}\text { Several additional symptoms can be present during the same } 2 \text { week period. } \\
\text { For a diagnosis of major depression, a total of at least } 5 \text { symptoms must be } \\
\text { present (including core symptoms). For a diagnosis of minor depression, } 2 \text { to } \\
4 \text { symptoms must be present. } \\
\text { - Appetite or weight disturbance, either: } \\
\text { - abnormal weight loss or decrease in appetite } \\
\text { - } \quad \text { abnormal weight gain or increase in appetite } \\
\text { - abnormal insomnia } \\
\text { - Abnormal fatigue or loss of energy } \\
\text { Activity disturbance (observable by others), either: } \\
\text { - abnormal agitation } \\
\text { - abnormal slowing } \\
\text { - Abnormal self-reproach or inappropriate guilt } \\
\text { - Abnormal poor concentration or indecisiveness }\end{array}$ \\
\hline C. & $\begin{array}{l}\text { The symptoms cause clinically important distress or impair work, social or } \\
\text { personal functioning }\end{array}$ \\
\hline
\end{tabular}

(Rankin, $\mathrm{p}=0.001$ ) and more cognitively disturbed (MMSE, $\mathrm{p}=0.04$ ) than patients of whom the GP was not interviewed (Table 1).

\section{METHODS}

\section{ASSESSMENT OF DEPRESSION DURING THE 1-YEAR FOLLOW-UP}

All patients were examined for the presence of a depressive episode at $1,3,6,9$, and 12 months after stroke. At the one-month assessment, all patients were intervicwed using the depression section of the Structured Clinical Interview for DSM-IV (SCID-1-R). This is a structured diagnostic psychiatric interview with a high inter-rater reliability, which is widely used. 132, 218 The Hamilton Depression Rating Scale (HAMD) was also scored as a measure for the severity of the 
depressive symptoms. ${ }^{96}$ At the remaining 4 assessments a screening method was used concerning of 3 self-rating scales. The Beck Depression Inventory (BDI), the Hospital Anxiety and Depression Scale (HADS, with 2 subscales for depression and anxiety), and the 90-items Symptom Checklist (SCL-90, with 8 subscales of which 1 for depression). ${ }^{69}, 129,136$ Patients were re-interviewed using the SCID and HAMD if the cut-off point of 1 of these scales was exceeded. The sensitivity of this method has been reported to be $92.2 \% .^{151}$ Depression was defined as major or minor depression according to DSM-IV criteria. ${ }^{47}$ For both diagnoses the presence of at least 1 core symptom of depression is needed (i.e. depressed mood and/or anhedonia), which is present almost permanently for at least 2 weeks. In the case of major depression, a total of 5 symptoms is needed, while in the case of minor depression, only 2 to 4 symptoms are needed (see Table 2). Diagnosis according to these criteria was considered to be the "gold standard".

\section{GP INTERVIEW}

Both patients and GPs were asked for permission before they were interviewed. An appointment for the structured interview by telephone was made with the receptionist. GPs were requested to use the notes of the patients' file involved when answering the questions. First, it was asked how many patient contacts there had been during the first year after the stroke (including home visits and consultations by telephone). Secondly, it was checked whether these consultations concerned mainly somatic, mental, other problems or a combination of these factors. All consultations in which mental or vague somatic problems were presented were discussed further one by one. At first, the interviewer asked about the nature of the complaint (somatic, mental or else) and whether mental complaints were discussed later during the consultation. Next, it was asked which diagnosis the GP had made and to give an estimate of the intensity (mild, moderate, severe), the course (sometimes, often, permanently) and the extent to which the patient was burdened by their complaints in his/her functioning. In order not to influence the GP the interviewer did not explicitly ask whether a diagnosis of depression was or was not made. It was asked which diagnosis the GP made regarding the psychic complaints that came up during the consultation. The mentioning or non-mentioning of depression as diagnosis was seen as the final result of the analysis. Furthermore, the GP was asked which treatment he/she had suggested and if a follow-up appointment had been made (no follow-up appointment, follow-up only if necessary, fixed appointment for follow-up). At last, the GP was asked for his general judgement concerning a change in the patients' mental functioning after stroke.

The mean time between the end of the 1-year follow-up period and the administration of the interview was $21.3 \pm 12.4$ weeks. 


\section{FACTORS THAT INFLUENCE RECOGNITION}

In order to determine which factors influenced adequate recognition of post-stroke depression by GPs, certain patient, consultation, and depression characteristics were studied. Patient characteristics were age, sex, level of education, personality traits, level of handicap, and psychiatric distress. These factors were measured one month after stroke. Personality was assessed using a self-rating scale that has been based on the 'Five Factor Model' of personality (NEO-FFI). ${ }^{159}$ Level of handicap was measured using the Rankin score. ${ }^{131}$ As a measure of psychological distress, the total score of the SCL-90 was used. Furthermore, cognitive functioning was measured globally using the Mini Mental State Examination (MMSE) and phasic disturbances were rated using the Frenchay Aphasia Screening Test (FAST). 128,153 Consultation characteristics concerned the number of consultations and the nature of the complaints that were presented by the patient. Depression characteristics concerned the differentiation between major and minor depression, the depression score on the SCL-90 and the duration of the depression, i.e. the number of times the diagnosis was made during the follow-up period.

\section{ANALYSIS}

For comparisons between patient groups (participants versus non-participants or depressed versus non-depressed patients) the Student's t-test was used in case of normally distributed, continuous variables and the non-parametric Mann Whitney U-Test was used in case of non-normally distributed data. The Chi-square test was used with categorical variables. Factors that possibly influenced adequate recognition of post-stroke depression by the GP were analysed using Logistic regression models. Except where age, sex, level of education, and level of handicap were concerned, the effects of these factors were tested one-sided because the hypothesised effects were clearly in one specific direction (for instance mild depression will be recognised less adequately and not vice versa). Because the follow-up assessments at 1, 3, 6, 9, and 12 months after stroke do not have to correspond with the GPs' patient contacts, the number of consultations and whether or not the GP was consulted when having psychic problems were considered as important determinants for adequate recognition of post-stroke deptession in this analysis.

\section{RESULTS}

The total number of consultations did not differ between depressed and nondepressed patients (median $6(1-50) ; \mathrm{p}=0.48$ ). Mental complaints, however, were 
TABLE 3. Correspondence between GP's and SCID diagnosis

\begin{tabular}{lccc}
\hline SCID diagnosis & \multicolumn{3}{c}{ GP's diagnosis } \\
\hline & Depressed & Not depressed & Total \\
Depressed & 11 & 18 & 29 \\
Not depressed & 3 & 26 & 29 \\
Total & 14 & 44 & 58 \\
\hline
\end{tabular}

found more often in depressed patients: $55.2 \%$ visited their GP at least once with primarily mental complaints, while another $24.1 \%$ discussed psychiatric complaints with their GP later during the consultation. In non-depressed patients this was only found in $27.6 \%$ and $20.7 \%$, respectively $(p=0,01)$.

Data concerning the recognition of depression by GPs are presented in table 3. In $37.9 \%(11 / 29)$ of depressed patients, the diagnosis was adequately recognised by the GP. In $89.7 \%(26 / 29)$ accordance was found concerning non-depressed patients.

Nine out of 29 depressed patients $(31.0 \%)$ were treated with an antidepressant, while two $(6.9 \%)$ were prescribed an anxiolytic drug. Ten patients were given a follow-up appointment, while one patient (3.4\%) was advised to return to the practise in case the complaints would persist or even aggravate (Table 4). Of those 14 patients in whom the GP diagnosed depression (11 true positives and 3 false positives), half were given antidepressants and one an anxiolytic drug. Seven were given a follow-up appointment, 2 only in case of persistence or aggravation of the symptoms. In all other cases, no antidepressant treatment was offered whatsoever (Table 4).

Bivariate analyses of factors that are responsible for the true or false recognition of depression by GPs showed, in the first place, that patients had rarely attended to their GP with primarily mental complaints in those cases in which depression stayed unrecognised. Furthermore, in these cases such complaints were

TABLE 4. Pharmacotherapy and follow-up by GPs

\begin{tabular}{lll}
\hline & SCID-diagnosis (n=29) & GP's diagnosis (n=14) \\
\hline No medication & $18(62,1 \%)$ & $6(42,9 \%)$ \\
Antidepressant* & $9(31,0 \%)$ & $7(50,0 \%)$ \\
Anxiolytic drug & $2(6,9 \%)$ & $1(7,1 \%)$ \\
\hline No folow-up arrangement & $18(62,1 \%)$ & $5(35,7 \%)$ \\
Concrete folow-up arrangement** & $10(34,5 \%)$ & $7(50,0 \%)$ \\
Follow-up only if necessary' ** & $1(3,4 \%)$ & $2(14,3 \%)$ \\
\hline
\end{tabular}

* with or without anxiolytic drug; ** at least once after diagnosis 
TABLE 5. Factors that influence the recognition of depression by the GP

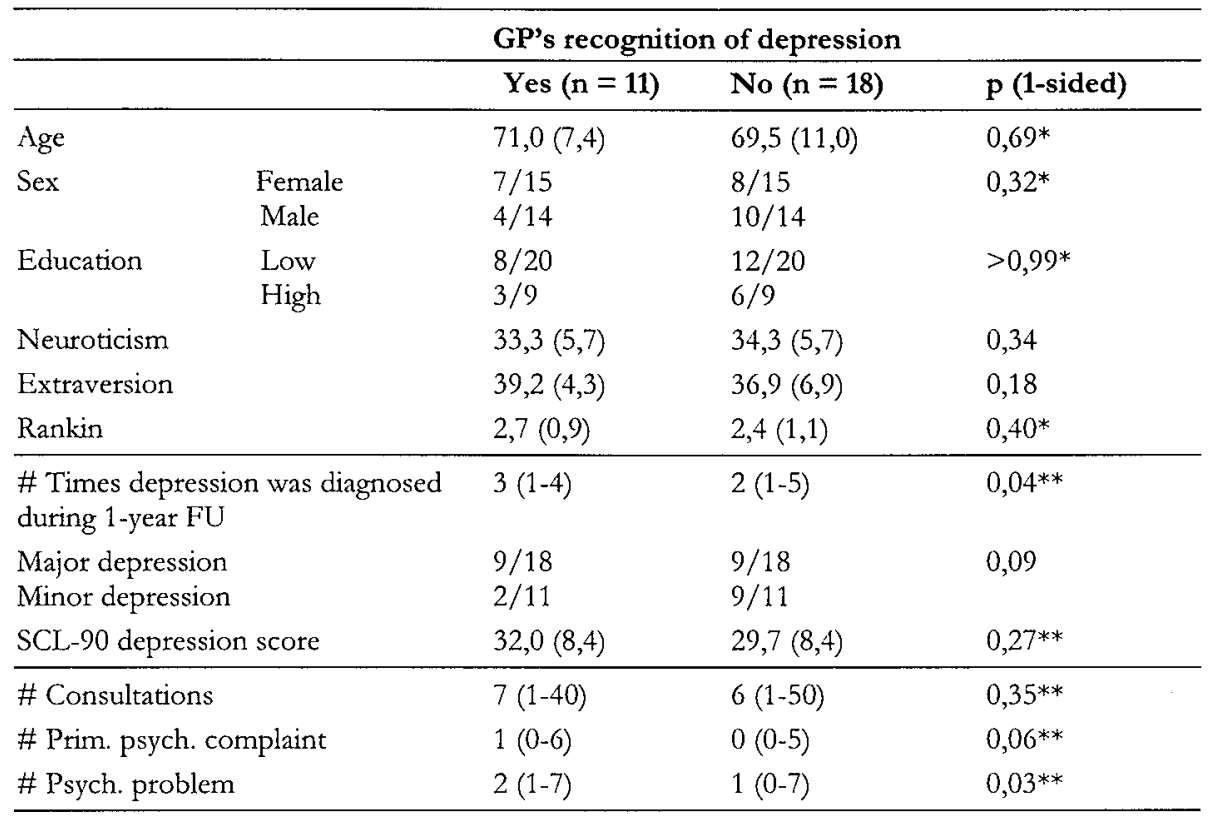

Rankin: handicap scale ( 0 no complaints -5 bedridden). '\#' means 'number of. \# Prim. psych. complaint: number of times that the patient consulted the GP with a primarily psych(iatr)ic complaint. \# Psych. problem: number of times that a psych(iatr)ic problem was discussed at any time during consultation. ${ }^{*}$ two-sided; ${ }^{* *}$ not corrected for ties.

infrequently discussed later during the consultation. In addition, depression that was recognised adequately was generally more severe than unrecognised depression. This was reflected by two variables: the nature and duration of the depression. Major depression was more often recognised than minor depression and depression that was diagnosed on repeated measurements during the 1-year follow-up was better recognised than depression that was detected at only one occasion during the follow-up period. The recognition of depression could not be related to patient characteristics such as age, sex, level of education, personality traits, or level of handicap (Table 5).

\section{DISCUSSION}

The recognition of post-stroke depression in this study was confined to a little less than $40 \%$. However, in the study of Loor et al. (1999) GPs recognised depression in $88.9 \%$ of patients who were also found to suffer from depression on the basis of 
a depression questionnaire as well. 219 This substantial difference may be due to differences in study design and research methods between both studies. First, major and minor depression in our study were diagnosed according to generally accepted diagnostic criteria and measured using a standardised diagnostic interview, while in the study by Loor (1999) it was only measured by using a self-rating questionnaire. Secondly, the recognition of depression by GPs may be underestimated in our study because the data from the GPs' notes were collected retrospectively and may have been incomplete. Even more important may be that GPs in Loors study had been asked more often about the presence of depression in their patients, which will have increased their sensitivity in recognising depression. The positive result of Loor must than be interpreted as evidence that the recognition of post-stroke depression can be substantially increased by reminding GPs of the problem of PSD regularly.

Our findings are more in accordance with studies that examined the recognition of depression in the elderly. Both Garrard (1998) and Crawford (1998) reported that in elderly patients depression was recognised in only $50 \%$ of cases. 205,206 In a Dutch study of Van Marwijk (1997) an even considerably lower percentage of $26 \%$ was found.207 Similar findings have been reported before by others. 209,210

Several factors seem to be responsible for the underrecognition of depression in the elderly. The symptom profile of depression in elderly patients has different characteristics compared to depression in the general population because of differences in the pathophysiology and the frequent occurrence of comorbidity. Depression often stays unnoticed because patients tend not to consult their GP with depressive complaints or mainly present physical complaints to their GP. This may apply to stroke patients even more, which was shown in our study, in which 13 of $29(45 \%)$ of depressed patients did not consult their GP with the essential complaint being of psychi(atri)c nature. The underrecognition of post-stroke depression by GPs must therefore at least partly be explained by the sickness behaviour of the patient.

In a number of cases, the GP did not specifically diagnose depression, while these patients were actually prescribed antidepressants. There may be different explanations for this: the antidepressants may have been prescribed by another physician (e.g. neurologist, rehabilitation doctor) or for another indication (e.g. anxiety disorder, neurogenic pain). Alternatively, the GP may have aimed to treat depressive symptoms without having made a well-defined diagnosis.

It can be discussed to which extent the diagnostic criteria of the DSM-IV can be applied validly in everyday practise. GPs will tend to pay more attention to the patients' problems and complaints than to check the presence or absence of all diagnostic criteria. Nevertheless, the DSM criteria used in this study, imply that depression was clinically relevant and accompanied by significant suffering or disability in everyday functioning. Because of this and because of the negative 
effects of depression on the morbidity and mortality after a stroke, adequate recognition and treatment of PSD seems to be important.

In conclusion, our results show that there is room for improvement where the role of GPs in the care and treatment of post-stroke patients is concerned. Stroke patients with depressive complaints rarely consult their GP. Therefore, the inventory of mental complaints in stroke patients, possibly by use of a short screening instrument, should be performed routinely in general practise. Such an instrument that is specifically designed for stroke patients is being tested by our group. A diagnosis of PSD should be an indication for treatment with antidepressants and/or supportive contacts, or for reference to a more specialised setting. To reach these goals, efforts should be made to develop ways to inform patients about PSD and other mental consequences of stroke, to develop standardised tools to measure mental problems in these patients and to develop continuing education for GPs and other health professionals who are working with stroke patients. 


\title{
CHAPTER 9 \\ General Discussion
}

\author{
Al haar geheimen \\ worden nu ze zijn ontbuld \\ geheimzinniger.
}

Nanneke Huiringa 


\section{GENERAL DISCUSSION}

In our current diagnostic systems (DSM-IV and ICD-10), psychiatric syndromes are classified without taking etiologic considerations into account. However, this does not apply to disorders with a known organic substrate. ${ }^{47,} 220$ The cardinal question of this thesis was, whether the relationship between stroke and depression is rather explained in specific cerebral terms, or can be seen as the result of multiple factors, including psychosocial ones, comparable with major depression in general. In this general discussion, an attempt is made to integrate the main results of our study with what is currently known about PSD and to explore their implications for both future research and clinical practice.

\section{METHODOLOGICAL ISSUES}

As discussed in chapter 2, research into post-stroke depression is complicated by a number of methodological difficulties. These have led to many inconsistent findings on the incidence and pathophysiology of PSD. These methodological issues regard, among others, lack of data prior to the occurrence of stroke, diagnostic instruments for depression that were not validated for use in stroke patients, discussion about proper diagnostic criteria for PSD, large heterogeneity between patients in stroke pathology, and differences in research designs concerning time of depression assessment and/or duration of follow-up. In addition, at the time this $\mathrm{PhD}$ project was initiated, there was a paucity of longitudinal studies with sufficient numbers of patients to enable multivariate analysis.

Although we could not overcome all of these problems, we have put effort into designing a reliable and accurate one-year follow-up study on PSD in 190 stroke patients with a first-ever supratentorial infarct. Along with a standardised diagnostic interview to diagnose PSD, depression self-rating scales were used to detect patients at risk. These self-rating scales were shown to be valid screening instruments in stroke patients. In addition, within the first month after stroke, a number of assessments were carried out such as CT and/or MR scans, personality assessments, rating scales for level of disability and handicap, expressed emotions by the caregiver, and cognitive functioning in order to study potential risk factors of biological, psychological or social nature.

We chose to confine our study to patients with a first-ever cerebral infarct with a supratentorial localisation and to exclude patients who were so severely disabled that they were not capable of participating in all assessments. This frequently concerned patients with severe aphasia or generalised cognitive disabilities. It is clear that this hampers the generalisability of our findings to the virtual population of all present and future stroke patients. It may be speculated that on average our 
cohort consists of patients with mild to mildly severe strokes. In a commentary on our comparative study of the incidence of depression after stroke vs. MI, TurnerStokes (2003) states that at present instruments are being developed to enable better assessment of depression in aphasic patients. ${ }^{221}$

Given the rather equal distribution of strokes over both hemispheres, it is not probable that exclusions have led to essential underrepresentation of right or left hemisphere strokes. Because of the relatively low frequency of infarcts with cortical involvement, of which a minority of $43 \%$ were located in the left hemisphere, it seems, however, that cortical strokes have been excluded relatively frequently, especially in the left hemisphere. One can imagine that patients with severe aphasia or generalised cognitive disabilities are especially vulnerable to depression, so that the cumulative incidence of PSD as reported in this thesis may be underestimated. Furthermore, we chose to combine major and minor depressions according to DSM-IV. This was done in accordance with other authors (see chapter 2), based on the notion that there is no principal reason for a strict distinction between minor and major depression, especially in the elderly population. ${ }^{180}$ Along with major depression, minor depression concerns an almost permanent disturbance of mood and/or hedonic functions lasting at least two weeks and causing personal suffering or dysfunctional behaviour in every day life. On the other hand, one may argue that major and minor depression are two distinct entities with different correlates. ${ }^{222}$ In our opinion and in line with others, we chose the position that there is no principal reason however, to discern the two conditions from each other.

As we followed the criteria of the DSM-IV, we assessed depression by asking patients about the core symptoms of depression (i.e. depressed mood and anhedonia) lasting at least two weeks. Therefore, diagnoses were primarily based on subjective experiences rather than the researchers observations of the patient's overt behaviour. Moreover, depression was diagnosed irrespective of the presence of other behavioural consequences that may occur after stroke, such as catastrophic or indifferent reactions, emotionalism, aprosody, apathy, et cetera. Most of these problems are poorly defined, unequivocal neuropsychiatric entities in themselves and valid and standardised instruments to diagnose these mental disturbances are mostly not present.

Another consequence of our design is that we were unable to assess the patient prior to the occurrence of his/her stroke. Therefore, we could only assess in retrospect if patients had not already been suffering from depression in the period precluding stroke. This also applies to premorbid vulnerability factors for depression, such as neuroticism. Personality assessment was performed at 1 month after stroke because stroke may alter one's personality or how one perceives $\mathrm{him} /$ herself. To enable such pre-stroke measurements, however, would demand inclusion of a large number of patients at risk of stroke like in epidemiologic, community-based studies with sample sizes of about 1000 and over. Of course, this 
would complicate the study seriously by prolonging its duration and raising the costs.

\section{INCIDENCE OF PSD}

Many different incidence and prevalence estimates of PSD have been reported in medical literature. These ranged broadly from $10-60 \%$, probably due to differences in study designs and methods. As a result, the magnitude of PSD as a medical problem stayed unclear. Therefore, in a literature review, we tried to divide studies on the basis of several study characteristics that may have contributed to these large differences in PSD frequency measures (chapter 2). Furthermore, we measured the one-year cumulative incidence of PSD in our cohort study of 190 first-ever stroke patients.

We reported that both major and minor depression occur frequently with incidence rates of $23 \%$ and $15 \%$ respectively (overall $38 \%$, see chapter 4 ). This fits reasonably well with the outcome of our descriptive review in which an overview was given of various incidence / prevalence reports on PSD taking into account patient setting, diagnostic instrument used to diagnose depression and time of assessment (chapter 2).

Most cases of PSD occurred already within 1 month, a finding that was also in line with results from other prospective follow-up studies.61, 94, 223 At 3, 6, 9, and 12 months after stroke the incidence rate stayed roughly the same (about $5 \%$ ) with a small tendency to increase over time. It has been reported that a second peak in incident cases occurs after 2-3 years. ${ }^{61}$ It was suggested that depression with an early-onset (1-3 months after stroke) must be distinguished from depression that develops later in the chronic course of stroke. Biological factors (i.e. lesion location) would be of more importance in early-onset PSD whereas psychosocial factors would be of more importance in late-onset PSD. ${ }^{174}$ However, we could not find any time-dependency in the relationship between PSD and the risk factors under study.

As mentioned in the above paragraph, the real incidence of PSD may even be higher than we have reported, since a substantial number of patients were excluded because of severe comorbidity, such as aphasia or generalised cognitive disabilities.

\section{RISK FACTORS AND PATHOPHYSIOLOGY}

The strong increase of depression incidence after stroke has often been emphasized to support the hypothesis that PSD has a specific pathophysiology, in which neurobiological alterations due to stroke also play a pathogenic role in depression. Indeed, PSD seemed to have a higher cumulative 1-year incidence than that seen in patients who were struck by a myocardial infarction (chapter 4). However, the reported ratio between PSD and post-MI depression was explained by differences 
in age, sex distribution, and level of handicap. Thus, the development of depression after stroke does not seem to be induced primarily by factors that are specifically related to stroke.

In line with this lack of support for the hypothesis that PSD is related to organic brain disease (i.e. stroke), we failed to establish a significant correlation between PSD and lesions located in the left hemisphere and/or in the anterior region of the brain (chapter 6). Several meta-analyses that were published during the last few years also concluded that there is insufficient evidence for the lesion location hypothesis to hold true.22-24 In reaction to these negative reports, Robinson stated in a recent review article that two meta-analyses that included time of onset of PSD in the analysis found significant correlations of left anterior infarcts and PSD in the first 2-6 months after stroke. ${ }^{174}$ In our data, however, we could not replicate such a time dependent effect.

Some recent findings revived the discussion in advantage of the lesion location hypothesis: Vataja et al. did not just look at the involvement of the frontal region but focused on lesions within the prefrontosubcortical circuitry. ${ }^{21}$ By using this strategy, they found an association between PSD and damage to this circuitry, especially in the left hemisphere. So, the lesion location hypothesis may not just be a matter of "anterior vs. posterior" but needs to be adjusted to the deterioration of structures that are involved in mood regulation processes. An explanatory model of neurobiological circuitry, opposed to the localisation tradition in neuroscience that was popular at the beginning of the twentieth century, is more in line with modern contemporary neuropsychiatry.

Furthermore, we failed to establish a significant effect of more generalised vascular brain damage (i.e. leukoaraiosis, silent cerebral infarcts) on the vulnerability to develop PSD (chapter 6). The explanation for this may hide in the powerful aspecific effects of stroke to induce depression, causing the relatively weak effect of white matter hyperintensities to be no longer detectable in a cohort of (only) 190 patients. It must be considered, however, that the negative findings on the lesion location and vascular depression hypothesis in our study may be the result of absence of MR data in the majority of cases. As a consequence, in these cases data on focal and generalised vascular damage had to be collected from CT scans made in the acute phase of stroke. No fresh infarct was visible on $45 \%$ of these scans, which is in line with several recent studies.(e.g. ${ }^{170}$ ) Furthermore, generalised vascular damage to the white matter is less sensitively but more specifically detected by CT than MR scans,. 224

Opposed to neurobiological risk factors, we found evidence for a robust effect of psychological factors. Premorbid neuroticism appeared to be of significant influence on the incidence of PSD. Neuroticism is a dimension of personality reflecting one's tendency to react to stressors with negative affective states such as anger, anxiety, sadness, etc (chapter 5). It has been shown to be a general 
vulnerability factor for depression. After stroke, which can be seen as an acute and powerful stressor, patients with high levels of neuroticism were almost five times more likely to develop PSD than were patients with low neuroticism. Similarly, the extent to which patients became handicapped after stroke independently increased the risk for depression. In addition, general risk factors such as female sex, prior history of depression, and family history of depression were significantly related to PSD in bivariate analysis but these effects were not independent from the handicap and/or neuroticism effect (chapter 7).

Overall, it can be concluded that stroke clearly increases the incidence of both major and minor depression but psychosocial ones outweigh the explanatory power of neurobiological factors. Now that solely biological explanatory models are clearly not unequivocally supported by research findings, more complex models including vulnerability factors, such as neuroticism and psychosocial stress factors, such as level of handicap need to be included. PSD can be considered as a challenge for modern neuropsychiatry and an exercise in applying the biopsychosocial model in its true sense in which different explanatory levels should be integrated.22.5

\section{RECOGNITION AND TREATMENT OF PSD}

Despite the known negative effects of depression on both the quality of life and life expectancy, only relatively few stroke patients were shown to visit their family doctor with mental complaints. This contributed to a low rate of recognition and treatment of post-stroke depression in general practices, a finding that opposes an earlier Dutch report on the recognition of PSD in general practice.219 It has important clinical implications since it has been shown that PSD can be effectively treated with antidepressants 174 and, even that prophylactic treatment of stroke patients with antidepressants significantly decreases the incidence of PSD and may therefore reduce loss of quality of life and early mortality.

A weakness of our study was that a diagnosis of depression by the GP was based on both the GP's notes and his own recollections, while the SCID diagnosis of depression used as the gold standard was administered in a 1-year prospective follow-up study. This might have contributed to the low rate of correspondence between the GP's judgement and the gold standard. Nevertheless, these findings underline the need for a more standardised approach in patients who have become more vulnerable after stroke.

\section{CLINICAL IMPLICATIONS}

The findings presented in this thesis support the notion that depression is a frequent comorbid psychiatric disorder following stroke that needs sufficient and adequate medical attention. Our results concerning the recognition and treatment of PSD in general practice indicate that this may be further improved. We 
recommend that every stroke patient should be assessed for depressive (and other psychiatric) symptomatology about 1-3 months after stroke, since in this period most cases of PSD have already emerged. In addition, patients and their caregivers benefit from early counselling in possible psychological stroke side-effects, which can help them to recognise symptoms in an early stage and make them aware that it is worth visiting their physician with these kinds of problems. We endorse the present development of multidisciplinary and transmural stroke units that have the expertise to diagnose and treat patients with PSD in a more standardised and evidence based manner than can be expected from family doctors.

Our findings further implicate that clinicians need to be wary of seeing PSD patients as only suffering from a pure organic psychosyndrome that results from an irreversible neurobiological defect. It urges the clinician to pay attention to the interplay between biological, psychological and social factors that can either act as premorbid (prior to stroke) vulnerability factors or as post-stroke stress factors. In figure 1 it has been attempted to visualise this multifactorial model, although consequent application of it raises problems as the interactions between the explanatory levels cannot be fully encompassed. Individual cases differ in the constellation of these factors and, therefore, need a treatment approach that is tailored to his/her individual situation. Some patients may need help in coping with their handicaps, while others need support in improving their social circumstances or need help with correcting their tendency to experience negative affects and have self-defeating ideas about their own functioning. Future studies should aim at developing a method to ascertain the value of such individually adjusted treatment approaches as well as the preventive power that goes from it.

FIGURE 1. Hypothesized model of PSD. The cumulative or interactive effects of both (biological, psychological, and social) consequences of stroke and premorbid (prior to stroke) vulnerability factors are suggested to cause depression after stroke.

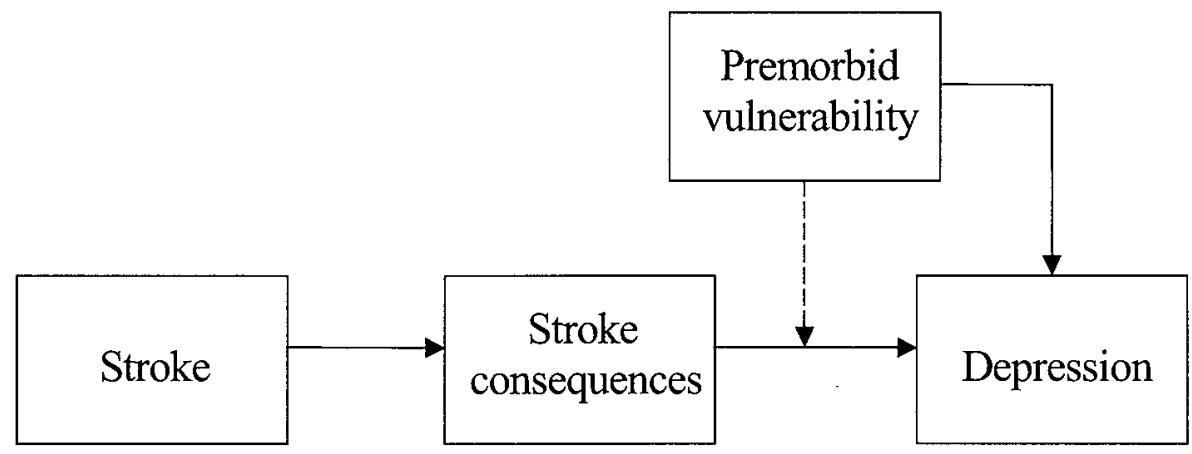




\section{RESEARCH IMPLICATIONS}

Our findings have implications for the pathophysiological concept of PSD. Since the early 1980s, this concept was dominated by findings of the American group of Robinson, who propagated that the location of the stroke lesion is crucial in the development of PSD. This was an appealing concept that led to enthusiastic research in this area. Although some authors have confirmed the role of lesions in the left hemisphere and/or in the anterior region of the brain, others failed to replicate these findings. In this still ongoing attempt to find a definite answer to the lesion location question, the influence of factors that are not specifically related to stroke have been relatively neglected. Our findings on the effect of neuroticism and level of handicap on the incidence of PSD imply that PSD should be considered to have a multifactorial origin including pre-stroke vulnerability and post-stroke stressors on psychosocial functioning. As stated in chapter 3 on the comparison of depression in stroke and MI patients, stroke should be considered an acute, lifethreatening event with consequences on biological (cerebral damage, functional deficits), psychological, and social functioning. The severity of these stressful consequences as well as one's pre-stroke vulnerability for depression can determine if PSD will finally occur (Figure 1). Generalised cerebrovascular damage, which has been shown to be a risk factor for depression in the elderly, does not seem to contribute significantly to the development of depression after stroke. This may be due to the greater impact of other factors that are, at least in part, of nonneurobiological nature, since depression in the course of other somatic diseases (e.g. MI) is also very common.

The primary challenge that PSD research faces is to generate results that can be replicated consistently and lead to unequivocal evidence. As illustrated in chapter 2 of this thesis, methodological problems seem to have caused the many inconsistent findings in PSD research during the last two decades. Therefore, it must be emphasized in the first place that effort should be put into improving the uniformity of study designs and methods. Consensus should be attained to define uniform diagnostic criteria, in which the in- or exclusion of somatic symptoms of depression is determined. More attention should be paid to the differentiation of depression from behavioural problems that can mimic depression such as aprosody and apathy. Minimal requirements about diagnostic instruments (e.g. standardisation, accuracy, validation in stroke population, etc.) also contribute to better comparability of studies. For this purpose, valid standardised diagnostic instruments need to be developed. Similarly, observational depression scales are warranted to allow patients with severe cognitive disabilities (including aphasia) to be included in studies on PSD, as was also emphasized by Turner-Stokes in her commentary to our study in the Lancet (2003).

In order to establish and further develop a differentiated multicausal model of PSD, large longitudinal studies are needed which have enough statistical power to 
allow inclusion of several interaction factors in multivariate analysis techniques. Only these kinds of studies are able to clarify the independent contribution of different vulnerability and stress factors in the pathophysiology of PSD. Furthermore, pathophysiological differences between major and minor depression and between early-onset and late-onset depression can only be detected in this way. With regards to the lesion location hypothesis and vascular depression hypothesis, the role of damage to the prefrontosubcortical circuitry, as shown by Vataja et al., still needs replication and further elaboration. The same goes for the few studies that looked at post-stroke lateralised differences in neurotransmitter receptor presentation. Functional imaging studies using techniques such as SPECT, PET, or functional MRI scans are necessary to relate brain damage in this region to disturbance in mood regulation in a more direct manner. For instance, it would be interesting to see if differences in frontal activity co-occur with differences in psychological modulation of affect (by looking at or listening to affectively loaded material). From this point of view, PSD research should adopt newly gained insights into the organisation of mood from basic neurosciences.

Other potential factors that influence the development of PSD also deserve to be further explored. First, cognitive dysfunction has mainly been studied as a risk factor for PSD by using the short and global MMSE. However, the cognitive apparatus consists of many specialised functions, which may interact with mood regulation to a different extent Retardation in the speed of cognitive processing or executive functioning, for instance is expected to have more impact than memory per se. Studies of this kind may help to bridge the gap between PSD and poststroke dementia, areas that at present seem too far away from each other.

Furthermore, the additional effect of certain psychological and social factors need further study. Different coping strategies, the intensity of expressed emotions by the carer, and the impact of stroke on one's social role are examples of such potential risk factors.

So far, PSD research has only looked at risk factors that can be measured in the first weeks after stroke. However, it is thinkable that characteristics of the course of stroke also influence the pathophysiology of PSD. For instance, the level of recovery of neurological deficits and the occurrence of new stressors, life events or physical morbidity may affect the development of PSD.

Finally, clinical PSD research must contribute to the development of optimal treatment strategies. As a consequence of the proposed multicausal origin of PSD, multidisciplinary treatment approaches should be developed in which interventions are adjusted to the individual constellation of depressogenic factors. Decision trees could serve to determine a patient's need for care in different domains. As a result, one patient could be offered ergotherapy and behavioural therapy, whilst in another strenghtening of the social system could be more favourable. Methods should be 
developed on how to evaluate such individually adjusted therapy programs. In addition, further research is needed to study the effectiveness and safety of antidepressant drugs. 


\section{References}

1. Konings-Dalstra J, Reitsma J. Hart- en vaatziekten in Nederland 1999, cijfers over ziekte en sterfte. Den Haag: Nederlandse Hartstichting.; 1999. Report No.: 90-75131-16-X.

2. Evers S, Struijs J, Ament A, et al. The disease impact, health care management, and costs of stroke in the Netherlands. Bilthoven (NL): RIVM; 2002. Report No.: 282701001.

3. Gupta A, Pansari K, Shetty H. Post-stroke depression. Int J Clin Pract 2002;56(7):531-537.

4. Mesulam M, editor. Principles of Behavioral and Cognitive Neurology. 2nd ed. Oxford: Oxford University Press, Inc.; 2000.

5. Gainotti G. Réactions "catastrophiques" et maniféstations d'indifférence au cours des atteintes cérébrales. Neuropsychologia 1969;7:195-204.

6. Chemerinski E, Robinson RG. The neuropsychiatry of stroke. Psychosomatics 2000;41(1):5-14.

7. Gainotti G, Caltagirone C, editors. Emotions and the dual brain. Berlin: Springer-Verlag; 1989.

8. Gainotti G. Emotional behavior and hemispheric side of the lesion. Cortex 1972;8(1):41-55.

9. Robinson RG, Price TR. Post-stroke depressive disorders: a follow-up study of 103 patients. Stroke 1982;13(5):635-641.

10. House A. Depression associated with stroke. J Neuropsychiatry Clin Neurosci 1996;8(4):453-457.

11. Goetz C, Pappert E, editors. Textbook of Clinical Neurology. 1st ed. Philadelphia (PA): W.B. Saunders Company; 1999.

12. Hochstenbach J. The cognitive, emotional and behavioural consequences of stroke $[\mathrm{PhD}$ thesis]. Nijmegen: Katholieke Universiteit Nijmegen; 1999.

13. Roman GC. Vascular dementia: distinguishing characteristics, treatment, and prevention. J Am Geriatr Soc 2003;51(5 Suppl Dementia):S296-304.

14. Erkinjuntti T, Rockwood K. Vascular dementia. Semin Clin Neuropsychiatry 2003;8(1):3745.

15. Rasquin S, Verhey FRJ, Van Oostenbrugge RJ, et al. Demographic and CT-scan features related to cognitive impairment in the first year after stroke. J Neurol Neurosurg Psychiatry 2004; (in press).

16. Castillo CS, Starkstein SE, Fedoroff JP, et al. Generalized anxiety disorder after stroke. J Nerv Ment Dis 1993;181(2):100-106.

17. Astrom M. Generalized anxiety disorder in stroke patients. A 3-year longitudinal study. Stroke 1996;27(2):270-275.

18. Robinson RG, Boston JD, Starkstein SE, et al. Comparison of mania and depression after brain injury: causal factors. Am J Psychiatry 1988;145(2):172-178.

19. Mayberg HS, Robinson RG, Wong DF, et al. PET imaging of cortical S2 serotonin receptors after stroke: lateralized changes and relationship to depression. Am J Psychiatry 1988;145(8):937-943.

20. Robinson RG. The clinical neuropsychiatry of stroke. Cognitive, behavioral and emotional disorders following vascular brain injury. 1st. ed. Cambridge: Cambridge University Press; 1998 
21. Vataja R, Pohjasvaara $T$, Leppavuori $A$, et al. Magnetic resonance imaging correlates of depression after ischemic stroke. Arch. Gen. Psychiatry 2001;58(10):925-931.

22. Singh $A$, Herrmann N, Black SE. The importance of lesion location in poststroke depression: a critical review. Can J Psychiatry 1998;43(9):921-927.

23. Carson AJ, MacHale S, Allen $K$, et al. Depression after stroke and lesion location: a systematic review. Lancet 2000;356:122-126.

24. Gainotti G, Marra C. Determinants and consequences of post-stroke depression. Curr Opin Neurol 2002;15(1):85-89.

25. Krishnan KR, Hays JC, Blazer DG. MRI-defined vascular depression. Am J Psychiatry 1997;154(4):497-501.

26. De Groot JC, De Leeuw FE, Oudkerk M, et al. Cerebral white matter lesions and depressive symptoms in elderly adults. Arch Gen Psychiatry 2000;57(11):1071-1076.

27. Morris PL, Robinson RG, Samuels J. Depression, introversion and mortality following stroke. Aust N Z J Psychiatry 1993;27(3):443-449.

28. Morris PL, Robinson RG, Andrzejewski P, et al. Association of depression with 10-year poststroke mortality. Am J Psychiatry 1993;150(1):124-129.

29. Robinson RG, Kubos KL, Starr LB, et al. Mood disorders in stroke patients. Importance of location of lesion. Brain 1984;107:81-93.

30. Mayou RA. Depression and type of physical disorder and treatment. In: Robertson M, Katona CLE, editors. Depression and physical illness. Chichester, West Sussex (UK): John Wiley \& sons Ltd; 1997. p. 21-38.

31. Agrell B, Dehlin O. Comparison of six depression rating scales in geriatric stroke patients. Stroke 1989;20(9):1190-1194.

32. Starkstein SE, Robinson RG. Affective disorders and cerebral vascular disease. $\mathrm{Br}$ J Psychiatry 1989;154:170-182.

33. House A. Mood disorders after stroke: $d$ review of the evidence. Int J Geriatr Psychiatry 1987;2:211-221.

34. Primeau F. Post-stroke depression: a critical review of the literature. Can J Psychiatry 1988;33(8):757-765.

35. Johnson GA. Research into psychiatric disorder after stroke: The need for further studies. Aust N Z J Psychiatry 1991;25:358-370.

36. Hosking SG, Marsh NV, Friedman PJ. Poststroke depression: prevalence, course, and associated factors. Neuropsychol Rev 1996;6(3):107-133.

37. Frühwald S, Löffler $H$, Baumhackl U. Depression nach zerebrovaskulären Ereignissen. Uberblick und Abgrenzung von anderen psychiatrischen Komplikationen. Fortschritte der Neurologie, Psychiatrie 1999;67(4):155-162.

38. Spencer KA, Tompkins CA, Schulz R. Assessment of depression in patients with brain pathology: the case of stroke. Psychol Bull 1997;122(2):132-152.

39. Rusin MJ. Stroke rehabilitation: a geropsychological perspective. Arch Phys Med Rehabil 1990;71(11):914-922.

40. Warlow CP, Dennis MS, Van Gijn J, et al. Stroke: a practical guide to management. 1st ed. Oxford: Blackwell Science Ltd; 1996.

41. Ricci S. Between-country variations in the use of medical treatments for acute stroke. In: Proceedings of 4th European Stroke Conference: Ccrebrovascular Disease; Bordeaux, France; 1995. p. 272.

42. Stroke Units Trialists' Collaboration. A systematic review of specialist multidisciplinary team (stroke unit) cate for stroke in-patients. In: Warlow C, van Gijn J, Sandercock P, 
editors. Stroke module of the Cochrane Database of systematic teviews. London: BMJ Publishing Group; 1996.

43. Chalmers GL. Post-stroke depression. Int Clin Psychopharmacol 1990;5(Suppl 3):21-31.

44. Paradiso S, Robinson RG. Minor depression after stroke: an initial validation of the DSMIV construct. Am J Geriatr Psychiatry 1999;7(3):244-251.

45. American Psychiatric Association. Diagnostic and Statistical Manual of Mental Disorders. 3rd ed. Washington (DC): American Psychiatric Press; 1980.

46. American Psychiatric Association. Diagnostic and Statistical Manual of Mental Disorders. 3rd, revised ed. Washington (DC): American Psychiatric Press; 1987.

47. American Psychiatric Association. Diagnostic and Statistical Manual of Mental Disorders. 4th. ed. Washington (DC): American Psychiatric Press; 1994.

48. Spitzer R, Endicott J, Robins E. Research Diagnostic Criteria (RDC) for a group of functional disorders. New York: Biometrics Research Division, New York Psychiatric Institute; 1975.

49. Robinson RG, Starkstein SE. Current research in affective disorders following stroke. J Neuropsychiatry Clin Neurosci 1990;2(1):1-14.

50. Fedoroff JP, Starkstein SE, Parikh RM, et al. Are depressive symptoms nonspecific in patients with acute stroke? Am J Psychiatry 1991;148(9):1172-1176.

51. Stein PN, Sliwinski MJ, Gordon WA, et al. Discriminative properties of somatic and nonsomatic symptoms for post stroke depression. The Clinical Neuropsychologist 1996;10(2):141-148.

52. Paradiso $S$, Ohkubo T, Robinson RG. Vegetative and psychological symptoms associated with depressed mood over the first two years after stroke. Int J Psychiatry Med 1997;27(2):137-157.

53. Endicott J. Measurement of depression in patients with cancer. Cancer 1984;53(10 Suppl):2243-2249.

54. Spitzer RL, Williams JB, Gibbon M, et al. The Structured Clinical Interview for DSM-III-R (SCID). I: History, rationale, and description. Arch Gen Psychiatry 1992;49(8):624-629.

55. Robins LN, Helzer JE, Croughan J, et al. National Institute of Mental Health Diagnostic Interview Schedule. Its history, characteristics, and validity. Arch Gen Psychiatry $1981 ; 38(4): 381-389$.

56. Tatemichi TK, Desmond DW, Stern Y, et al. Cognitive impairment after stroke: frequency, patterns, and relationship to functional abilities. J Neurol Neurosurg Psychiatry 1994;57(2):202-207.

57. Verhey FRJ, Visser PJ. The phenomenology of depression in dementia. Int Psychogeriatr 2000;12:129-134.

58. Gordon WA, Hibbard MR, Egelko S, et al. Issues in the diagnosis of post-stroke depression. Rehabil Psychol 1991;36(2):71-87.

59. Black KJ. Diagnosing depression after stroke. South Med J 1995;88(7):699-708.

60. Dam H, Pedersen HE, Ahlgren P. Depression among patients with stroke. Acta Psychiatr Scand 1989;80(2):118-124.

61. Astrom M, Adolfsson R, Asplund K. Major depression in stroke patients. A 3-year longitudinal study. Stroke 1993;24(7):976-982.

62. Schwartz JA, Speed NM, Brunberg JA, et al. Depression in stroke rehabilitation. Biol. Psychiatry 1993;33(10):694-699.

63. Angeleri F, Angeleri VA, Foschi N, et al. Depression after stroke: An investigation through catamnesis. J Clin Psychiatry 1997;58(6):261-265. 
64. Hermann $M$, Bartels C, Schumacher $M$, et al. Poststroke depression. Is there a pathoanatomic correlate for depression in the postacute stage of stroke? Stroke 1995;26(5):850-856.

65. Kauhanen M, Korpelainen JT, Hiltunen P, et al. Poststroke depression correlates with cognitive impairment and neurological deficits. Stroke 1999;30(9):1875-1880.

60. Gainoti G, Azzoni A, Marra C. Frcquency, phenomenology and anatomical-clinical correlates of major post-stroke depression. Br J Psychiatry 1999;175:163-167.

67. Zung WW. A sclf-rating depression scale. Arch. Gen. Psychiatry 1965;12(1):63-70.

68. Radloff I.S. The CES-D Scale: $\Lambda$ self-report depression scale for research in the general population. Applied Psychological Measurement 1977;1 (3):385-401.

69. Beck $\mathrm{AT}, \mathrm{W}$ ard $\mathrm{CH}$, Mendelson $\mathrm{M}$, et al. An Inventory for measuring depression. Arch Gen Psychiatry 1961;4:561-571.

70. Sinyor D, Amato P, Kaloupck DG, et al. Post-stroke depression: relationships to functional impairment, coping stratcgics, and rehabilitation outcome. Stroke 1986;17(6):1102-1107.

71. Ebrahim S, Barer D, Nouri F. Affecoive illness after stroke. Br J Psychiatry 1987;151:52-56.

72. Wade DT, Legh-Smith J, Hewer R $\lambda$. Depressed mood after stroke. $A$ community study of its frequency. Br. J. Psychiatry 1987;151:200-205.

73. Morris PL, Robinson RG, De Carvalho ML, et al. Lesion characteristics and depressed mood in the stroke data bank study. J Neuropsychiatry Clin Neurosci 1996;8(2):153-159.

74. Fuh JL, Liu HC, Wang SJ, et al. Poststroke depression among the Chincsc elderly in a rural community. Stroke 1997;28(6):1126-1129.

75. Beekman AT, Penninx BW, Decg DJ, et al. Depression in survivors of stroke: a community-based study of prevalence, risk factors and consequences. Soc Psychiatry Psychiatr Epidemiol 1998;33(10):463-470.

76. Herrmann N, Black SE, Lawrence J, et al. The Sunnybrook Stroke Study: a prospective study of depressive symptoms and functional outcomc. Stroke 1998;29(3):618-624.

77. Kotila $\mathrm{M}$, Numminen $\mathrm{H}$, Waltimo $\mathrm{O}$, et al. Depression after stroke: results of the FINNSTROKE Study. Stroke 1998;29(2):368-372.

78. Ramasubbu R, Robinson RG, Flint AJ, et al. Functional impairment associated with acute poststroke depression: the Stroke Data Bank Study. J Neuropsychiatry Clin Neurosci 1998;10(1):26-33.

79. Shinar D, Gross CR, Price TR, et al. Screening for depression in stroke patients: the reliability and validity of the Center for Epidemiologic Studies Depression Scale. Strokc 1986;17(2):241-245.

80. House $A$, Dennis $M$, Hawton $K$, et al. Methods of identifying mood disorders in stroke patients: experience in the Oxfordshire Community Stroke Project. Age Ageing $1989 ; 18(6): 371-379$.

81. Johnson G, Burvill PW, Anderson C.S, et al. Screening instruments for depression and anxiety following stroke: experience in the Perth community stroke study. Acta Psychiatr. Scand. 1995;91(4):252-257.

82. Rybarczyk B, Winemiller DR, Lazarus I.W, et al. Validation of a depression screening measure for stroke inpatients. Am J Geriatr Psychiatry 1996;4:131-139.

83. O'Rourke S, MacHalc S, Signorini D, et al. Detecting psychiatric morbidity after stroke: comparison of the GHQ and the HAD Scale. Stroke 1998;29(5):980-985.

84. Sliwinski M, Gordon WA, Bogdany J. The Beck Depression Inventory: is it a suitable measure of depression for individuals with traumatic brain injury? J Head Trauma Rehabil 1998;13(4):40-46. 
85. Eaton WW, Neufeld K, Chen LS, et al. A comparison of self-report and clinical diagnostic interviews for depression: Diagnostic Interview Schedule and schedules for clinical assessment in neuropsychiatry in the Baltimore Epidemiologic Catchment Area follow-up. Arch Gen Psychiatry 2000;57(3):217-222.

86. Hibbard MR, Gordon WA, Stein PN, et al. A multimodal approach to the diagnosis of poststroke depression. In: Gordon WA, editor. Advances in stroke rehabilitation. Boston: Andover Medical; 1993. p. 185-214.

87. Sutcliffe LM, Lincoln NB. The assessment of depression in aphasic stroke patients: the development of the Stroke Aphasic Depression Questionnaire. Clin Rehabil 1998;12(6):506-513.

88. Gainotti G, Azzoni A, Razzano C, et al. The Post-Stroke Depression Rating Scale: a test specifically devised to investigate affective disorders of stroke patients. J Clin Exp Neuropsychol. 1997;19(3):340-356.

89. Nelson LD, Satz $P$, Mitrushina $M$, et al. Development and validation of the Neuropsychology Behavior and Affect Profile. Psychological Assessment: a journal of consulting and clinical psychology 1989;1:266-272.

90. Robinson RG, Starr LB, Lipsey JR, et al. A two-year longitudinal study of post-stroke mood disorders: dynamic changes in associated variables over the first six months of follow-up. Stroke 1984;15(3):510-517.

91. Robinson RG, Start LB, Price TR. A two year longitudinal study of mood disorders following stroke. Prevalence and duration at six months follow-up. $\mathrm{Br} J$ Psychiatry 1984;144:256-262.

92. Robinson RG, Lipsey JR, Rao K, et al. Two-year longitudinal study of post-stroke mood disorders: comparison of acute-onset with delayed-onset depression. Am J Psychiatry 1986;143(10):1238-1244.

93. Lipsey JR, Spencer WC, Rabins PV, et al. Phenomenological comparison of poststroke depression and functional depression. Am J Psychiatry 1986;143(4):527-529.

94. Andersen G, Vestergaard $\mathrm{K}$, Riis J, et al. Incidence of post-stroke depression during the first year in a large unselected stroke population determined using a valid standardized rating scale. Acta Psychiatr. Scand. 1994;90(3):190-195.

95. Fedoroff JP, Lipsey JR, Starkstein SE, et al. Phenomenological comparisons of major depression following stroke, myocardial infarction or spinal cord lesions. J Affect Disord. 1991;22(1-2):83-89.

96. Hamilton M. A rating scale for depression. J Neurol Neurosurg Psychiatry 1960;23:56-62.

97. Katon W, Schulberg H. Epidemiology of depression in primary care. Gen Hosp Psychiatry 1992;14(4):237-247.

98. Enst C, Angst J. The Zurich Study. XII. Sex differences in depression. Evidence from longitudinal epidemiological data. Eur Arch Psychiatry Clin Neurosci 1992;241(4):222-230.

99. Myers JK, Weissman MM, Tischler GL, et al. Six-month prevalence of psychiatric disorders in three communities 1980 to 1982. Arch Gen Psychiatry 1984;41(10):959-967.

100. House A., Dennis M, Warlow C, et al. Mood disorders after stroke and their relation to lesion location. A CT scan study. Brain 1990;113:1113-1129.

101. House A, Dennis M, Mogridge L, et al. Mood disorders in the year after first stroke. Br J Psychiatry 1991;158:83-92.

102. Burvill PW, Johnson GA, Jamrozik KD, et al. Prevalence of depression after stroke: the Perth Community Stroke Study. Br J Psychiatry 1995;166(3):320-327.

103. Burvill P, Johnson G, Jamrozik $K$, et al. Risk factors for post-stroke depression. Int J Geriatr Psychiatry 1997;12(2):219-226. 
104. Robinson RG, Starr LB, Kubos KL, et al. A two-year longirudinal study of post-stroke mood disorders: findings during the initial evaluation. Stroke 1983;14(5):736-741.

105. Downhill JE, Jr., Robinson RG. Longitudinal assessment of depression and cognitive impairment following stroke. J Nerr Ment Dis 1994;182(8):425-431.

106. Starkstein SE, Robinson RG, Honig MA, et al. Mood changes after right-hemisphere lesions. Br J Psychiatry 1989;155:79-85.

107. Pohjasvaara T, Leppavuori A, Siira I, et al. Frequency and clinical determinants of poststroke depression. Stroke 1998;29(11):2311-2317.

108. Morris PL, Robinson RG, Raphael B. Prevalence and course of depressive disorders in hospitalized stroke patients. Int J Psychiatry Med 1990;20(4):349-364.

109. Eastwood MR, Rifat SL, Nobbs H, et al. Mood disorder following cerebrovascular accident. Br J Psychiatry 1989;154:195-200.

110. Morris PL, Robinson RG, Raphael B, et al. The relationship between risk factors for affective disorder and poststroke depression in hospitalised stroke patients. Aust $\mathrm{N} \mathrm{Z} \mathrm{J}$ Psychiatry 1992;26(2):208-217.

111. Spencer KA, Tompkins CA, Schulz R, th al. A longitudinal study of depression risk. Clinic Aphasiol 1995;23:9-23.

112. Robinson RG, Bolduc PL, Price TR. Two-year longitudinal study of poststroke mood disorders: diagnosis and outcome at one and two years. Stroke 1987;18(5):837-843.

113. Gordon WA, Hibbard MR. Poststroke depression: an examination of the literature. Arch Phys Med Rehabil 1997;78(6):658-663.

114. Robinson RG, Kubos KL, Starr LB, et al. Mood changes in stroke patients: relationship to lesion location. Compr Psychiatry 1983;24(6):555-566.

115. Starkstein SE, Robinson RG, Price TR. Comparison of cortical and subcortical lesions in the production of poststroke mood disorders. Brain 1987;110:1045-1059.

116. Alexopoulos GS, Meyers BS, Young RC, et al. 'Vascular depression' hypothesis. Arch Gen Psychiatry 1997;54(10):915-922.

117. Carney RM, Rich MW, Tevelde A, et al. Major depressive disorder in coronary artery disease. Am J Cardiol 1987;60(16):1273-1275.

118. Rabkin JG, Charles E, Kass F. Hypertension and DSM-III depression in psychiatric outpatients. Am J Psychiatry 1983;140(8):1072-1074.

119. Fujikawa $\mathrm{T}$, Yamawaki $\mathrm{S}$, Touhouda $\mathrm{Y}$. Incidence of silent cerebral infarction in patients with major depression. Stroke 1993;24(11):1631-1634.

120. Bryan RN, Manolio TA, Schertz LD, et al. A method for using MR to evaluate the effects of cardiovascular disease on the brain: the Cardiovascular Health Study. Amcrican Journal of Neuroradiology 1994;15(9):1625-1633.

121. Braffman BH, Zimmerman RA, Trojanowski JQ, et al. Brain MR: pathologic correlation with gtoss and histopathology. 2. Hyperintense white-matter foci in the elderly. Am J Roentgenol 1988;151(3):559-566.

122. Krishnan KR, Goli V, Ellinwood EH, et al. Leukoencephalopathy in patients diagnosed as major depressive. Biol Psychiatry 1988;23(5):519-522.

123. Baldwin RC, Tomenson B. Depression in later life. A comparison of symptoms and risk factors in early and late onset cases. Br J Psychiatry 1995;167(5):649-652.

124. Fujikawa T, Yamawaki S, Touhouda Y. Background factors and clinical symptoms of major depression with silent cerebral infarction. Stroke 1994;25(4):798-801.

125. House A. Mood disorders in the first year after stroke. Nurs Times 1991;87(15):53-54. 
126. Kotila $\mathrm{M}$, Numminen $\mathrm{H}$, Waltimo $\mathrm{O}$, et al. Post-stroke depression and functional recovery in a population-based stroke register: The Finnstroke study. Eur J Neurol 1999;6(3):309312.

127. Boon A, Lodder J, Heuts-van Raak $L$, et al. Silent brain infarcts in 755 consecutive patients with a first-ever supratentorial ischemic stroke. Relationship with index-stroke subtype, vascular risk factors, and mortality. Stroke 1994;25(12):2384-2390.

128. Folstein MF, Folstein SE, McHugh PR. 'Mini-Mental State': a practical method for grading the cognitive state of parients for the clinician. J Psychiatr Res 1975;12(3):189-198.

129. Zigmond AS, Snaith RP. The Hospital Anxiety and Depression Scale. Acta Psychiatr Scand 1983;67(6):361-370.

130. Mahoney FI, Barthel DW. Functional evaluation: the Barthel Index. State Medical Journal $1965 ; 14: 61$.

131. Rankin J. Cerebral vascular accidents in patients over the age of 60. 2. Prognosis. Scott Med J 1957;2:200-215.

132. First MB, Spitzer RL, Gibbon M, et al. Structured Clinical Interview for DSM-IV $A$ xis I Disorders - Patient Edition (SCID-I/P, Version 2.0). New York (NY): Biometrics Research Department, New York State Psychiatric Institute; 1995.

133. Richter $P$, Werner J, Heerlein $A$, et al. On the validity of the Beck Depression Inventory, $A$ review. Psychopathology 1998;31(3):160-168.

134. Herrmann C. International experiences with the Hospital Anxiety and Depression Scale -a review of validation data and clinical results. J Psychosom Res 1997;42(1):17-41.

135. Derogatis LR, Lipman RS, Covi L. SCL-90: an outpatient psychiatric rating scalepreliminary report. Psychopharmacol Bull 1973;9:13-27.

136. Arrindell WA, Ettema JHM. Dimensionele structuur, betrouwbaatheid en validiteit van de Nederlandse bewerking van de Symptom Checklist (SCL-90). Ned Tijdschr Psychologie. 1981;43:381-387.

137. Woessner R, Caplan B. Emotional distress following stroke: Interpretive limitations of the SCL-90-R. Assessment 1996;3(3):291-305.

138. Honig A, Lousberg $R$, Wojchiechowski $F$, et al. Depressie na een eerste hartinfarct: overeenkomsten en verschillen met een gewone depressie. Ned. Tijdschr. Geneeskunde 1997;141:196-199.

139. Murphy JM, Berwick DM, Weinstein MC, et al. Performance of screening and diagnostic tests. Application of Receiver Operating Characteristic analysis. Arch Gen Psychiatry 1987;44(6):550-555.

140. Mirowsky J, Ross CE. Sex differences in distress: real or artifact? Am Sociol Rev 1995;60:449-468.

141. Aho K, Harmsen P, Hatano S, et al. Cerebrovascular disease in the community: results of a WHO collaborative study. Bulletin of the World Health Organization 1980;58(1):113-130.

142. Bamford J, Sandercock P, Dennis M, et al. A prospective study of acute cerebrovascular disease in the community: the Oxfordshire Community Stroke Project 1981-86. 1. Methodology, demography and incident cases of first-ever stroke. J Ncurol Neurosurg Psychiatry 1988;51(11):1373-1380.

143. Leentjens AF, Verhey FR, Lousberg $\mathrm{R}$, et al. The validity of the Hamilton and Montgomery- $A$ sberg depression rating scales as screening and diagnostic tools for depression in Parkinson's disease. Int J Geriatr Psychiatry 2000;15(7):644-649.

144. Morris PL, Robinson RG. Personality neuroticism and depression after stroke. Int J Psychiatry Med 1995;25(1):93-102. 
145. Morris PL, Robinson RG, Raphael B, et al. The relationship between the perception of social support and post-stroke depression in hospitalized patients. Psychiatry 1991;54(3):306-316.

146. Robinson RG, Murata $Y$, Shimoda K. Dimensions of social impairment and their effect on depression and recovery following stroke. Int Psychogeriatr 1999;11(4):375-384.

147. Rao R, Jackson S, Howard R. Depression in older people with mild stroke, carotid stenosis and peripheral vascular disease: A comparison with healthy controls. Int J Geriatr Psychiatry 2001;16(2):175-183.

148. Murphy MT. The epidemiology of depression in acute stroke and cardiac in-patients [PhD thesis]. Pennsylvania: The Pennsylvania State University; 1996.

149. National Institute of Neurological Disorders and Stroke. Special report from the National Institute of Neurological Disorders and Stroke. Classification of cerebrovascular diseases III. Stroke 1990;21(4):637-676.

150. Pasternak RC, Braunwald E, Sobel RE. Acute myocardial infarction. In: Braunwald E, editor. Heart disease: a textbook of cardiovascular medicine. 4th. ed. Philadelphia: W. B. Saunders Compagny; 1992. p. 1200-1291.

151. Aben I, Verhey F, Lousberg R, et al. Validity of the Beck Depression Inventory, Hospital Anxiety and Depression Scale, SCL-90, and Hamilton Depression Rating Scale as screening instruments for depression in stroke patients. Psychosomatics 2002;43(5):386-393.

152. Strik J, Honig A, Lousberg $R$, et al. Sensitivity and specificity of observer and self-report questionnaires in major and minor depression following myocardial infarction. Psychosomatics 2001;42(5):423-428.

153. Enderby PM, Wood VA, Wade DT, et al. The Frenchay Aphasia Screening Test: a short, simple test for aphasia appropriate for non-specialists. Int Rehabil Med 1987;8(4):166-170.

154. May M, McCarron P, Stansfeld S, et al. Does psychological distress predict the risk of ischemic stroke and transient ischemic attack? The Caerphilly Study. Stroke 2002;33(1):712.

155. Carney RM, Freedland KE. Psychological distress as a risk factor for stroke-related mortality. Stroke 2002;33(1):5-6.

156. Costa PT, Jr., McCrae RR. The NEO Personality Inventory Manual. Odessa, Flotida: Psychological Assessment Resources Inc.; 1985.

157. Watson D, Clark LA, Carey G. Positive and negative affectivity and their relation to anxiety and depressive disorders. J Abnorm Psychol 1988;97(3):346-353.

158. First MB, Gibbon M, Spitzer RL, et al. Users Guide for the Structured Clinical Interview for DSM-IV Axis I Disorders - Research Version - (SCID-I, Version 2.0). New York (NY): New York State Psychiatric Institute; 1996.

159. Hoekstra HA, Ormel J, De Fruyt F. NEO Personality Questionnaires NEO-PI-R, NEOFFI, Manual. Lisse: Swets \& Zeitlinger B.V.; 1996.

160. Enns MW, Cox BJ. Personality dimensions and depression: Review and commentary. Can J Psychiatry 1997;42(3):274-284.

161. Aben I, Verhey F, Honig A, et al. Research into the specificity of depression after stroke: a review on an untesolved issue. Progr Neuropsychopharm Biol Psychiatry 2001;25(4):671689.

162. Hirschfeld RMA, Shea MT, Holzer III CE. Personality dysfunction and depression. In: Honig A, Van Praag HM, editors. Depression. Neurobiological, psychopathological and therapeutic advances. Chichester, West Sussex (UK): John Wiley \& Sons Ltd; 1997. p. 658.

163. Bagby RM, Rector NA, Bindseil K, et al. Self-report ratings and informants' ratings of personalities of depressed outpatients. Am J Psychiatry 1998;155(3):437-438. 
164. Narushima K, Kosier JT, Robinson RG. A reappraisal of poststroke depression, intra- and inter-hemispheric lesion location using meta-analysis. J Ncuropsychiatry Clin Neurosci 2003;15(4):422-430.

165. Honig A, Van Praag HM, editors. Depression. Neurobiological, psychopathological and therapeutic advances. Chichester, West Sussex (UK): John Wiley \& Sons; 1997.

166. Robertson MM, Katona CLE, editors. Depression and physical illness. Chichester, West Sussex (UK): Wiley; 1997.

167. Aben I, Denollet J, Lousberg R, et al. Personality and vulnerability to depression in stroke patients: a 1-year prospective follow-up study. Stroke 2002;33(10):2391-2395.

168. Boreas AM, Lodder J, Kessels F, et al. Prognostic value of blood pressure in acute stroke. J Hum Hypertens 2002;16(2):111-116.

169. De Jong G, Van Raak L, Kessels F, et al. Stroke subtype and mortality. a follow-up study in 998 patients with a first cerebral infarct. J Clin Epidemiol 2003;56(3):262-268.

170. Wardlaw JM, West TM, Sandercock PA, et al. Visible infarction on computed tomography is an independent predictor of poor functional outcome after stroke, and not of haemorrhagic transformation. J Neurol Neurosurg Psychiatry 2003;74(4):452-458.

171. Hupperts RM, Lodder J, Heuts-van Raak EP, et al. Infarcts in the anterior choroidal artery territory. Anatomical distribution, clinical syndromes, presumed pathogenesis and early outcome. Brain 1994;117 (Pt 4):825-834.

172. Inzitari D. Leukoaraiosis: an independent risk factor for stroke? Stroke 2003;34(8):20672071.

173. Fazekas F, Chawluk JB, Alavi A, et al. MR signal abnormalities at $1.5 \mathrm{~T}$ in Alzheimer's dementia and normal aging. Am J Roentgenol 1987;149(2):351-356.

174. Robinson RG. Poststroke depression: prevalence, diagnosis, treatment, and disease progression. Biol Psychiatry 2003;54(3):376-387.

175. Mast BT, MacNeill SE, Lichtenberg PA. Post-stroke and clinically-defined vascular depression in geriatric rehabilitation patients. Am J Geriatr Psychiatry 2004;12(1):84-92.

176. Mast BT, Neufeld S, MacNeill SE, et al. Longitudinal support for the relationship between vascular risk factors and late-life depressive symptoms. Am J Geriatr Psychiatry 2004;12(1):93-101.

177. Beekman AT, Copeland JR, Prince MJ. Review of community prevalence of depression in later life. Br J Psychiatry 1999;174:307-311.

178. King RB. Quality of life after stroke. Stroke 1996;27(9):1467-1472.

179. Kim P, Warren S, Madill H, et al. Quality of life of stroke survivors. Quality of Life Research: An International Journal of Quality of Life Aspects of Treatment, Care and Rehabilitation 1999;8(4):293-301.

180. Beekman ATF, Deeg DJH, Van Tilburg T, et al. Major and minor depression in later life: a study of prevalence and associated factors. J Affect Disord 1995;36:65-75.

181. Schoevers RA, Beekman AT, Deeg DJ, et al. Risk factors for depression in later life; results of a prospective community based study (AMSTEL). J Affect Disord 2000;59(2):127-137.

182. Beekman AT, Deeg DJ, Geerlings SW, et al. Emergence and persistence of late life depression: a 3-year follow-up of the Longitudinal Aging Study Amsterdam. J Affect Disord 2001;65(2):131-138.

183. Stordal E, Bjartveit Kruger M, Dahl NH, et al. Depression in relation to age and gender in the general population: the Nord-Trondelag Health Study (HUNT). Acta Psychiatr Scand 2001;104(3):210-216. 
184. Lindeman S, Hamalainen J, Isometsa E, et al. The 12-month prevalence and risk factors for major depressive episode in Finland: representative sample of 5993 adults. Acta Psychiatr Scand 2000;102(3):178-184.

185. Forsell Y, Winblad B. Major depression in a population of demented and nondemented older people: prevalence and correlates. J Am Geriatr Soc 1998;46(1):27-30.

186. Andersen $G$, Vestergaard $K$, Ingemann-Nielsen $M$, et al. Risk factors for post-stroke depression. Acta Psychiatr Scand 1995;92(3):193-198.

187. Kim JS, Choi Kwon S. Poststroke depression and emotional incontinence: Correlation with lesion location. Neurology 2000;54(9):1805-1810.

188. Shimoda K, Robinson RG. The relationship between poststroke depression and lesion location in long-term follow-up. Biol Psychiatry 1999;45(2):187-192.

189. Stewart R, Prince M, Richards M, et al. Stroke, vascular risk factors and depression: Crosssectional study in a UK Caribbean-born population. Br J Psychiatry 2001;178(1):23-28.

190. Aben I, Verhey F, Strik J, et al. A comparative study into the one year cumulative incidence of depression after stroke and myocardial infarction. J Neurol Neurosurg Psychiatry 2003;74(5):581-585.

191. Paradiso S, Robinson RG. Gender differences in poststroke deptession. J Neuropsychiatry Clin Neurosci 1998;10(1):41-47.

192. Sonnenberg CM, Beekman AT, Deeg DJ, et al. Sex differences in late-life depression. Acta Psychiatr Scand 2000;101(4):286-292.

193. Morris PLP, Robinson RG, Raphael B, et al. The relationship between risk factors for affective depression in hospitalised stroke patients. Aust N Z J Psychiatry 1992;26(2):208217.

194. Sharpe M, Hawton K, Seagroatt V, et al. Depressive disorders in long-term survivors of stroke. Associations with demographic and social factors, functional status, and brain lesion volume. Br J Psychiatry 1994;164(3):380-386.

195. Morris PL, Raphael B, Robinson RG. Clinical depression is associated with impaired recovery from stroke. Med J Aust 1992;157(4):239-242.

196. Shima S, Kitagawa Y, Kitamura T, et al. Poststroke depression. Gen Hosp Psychiatry 1994;16(4):286-289.

197. Cole MG, Dendukuri N. Risk factors for depression among elderly community subjects: a systematic review and meta-analysis. Am J Psychiatry 2003;160(6):1147-1156.

198. Katon WJ. Clinical and health services relationships between major depression, depressive symptoms, and general medical illness. Biol Psychiatry 2003;54(3):216-226.

199. Cole SA, Woodard JL, Juncos JL, et al. Depression and disability in Parkinson's disease. J Neuropsychiatry Clin Neurosci 1996;8(1):20-25.

200. Leentjens AF, Lousberg R, Verhey FR. Markers for depression in Parkinson's disease. Acta Psychiatr Scand 2002;106(3):196-201.

201. McEwen BS. Mood disorders and allostatic load. Biol Psychiatry 2003;54(3):200-207.

202. Starkstein SE, Robinson RG. Neuropsychiatric aspects of cerebral vascular disorders. In: Yudofsky SC, Hales RE, editors. The American Psychiatric Press Textbook of Neuropsychiatry. 2nd ed. Washington (DC): Ametican Psychiatric Press; 1992. p. 449-472.

203. Wiart L, Petit H, Joseph PA, et al. Fluoxetine in early poststroke depression: a double-blind placebo-controlled study. Stroke 2000;31(8):1829-1832.

204. Robinson RG, Schultz SK, Castillo C, et al. Nortriptyline versus fluoxetine in the treatment of depression and in short-term recovery after stroke: a placebo-controlled, double-blind study. Am J Psychiatry 2000;157(3):351-359. 
205. Garrard J, Rolnick SJ, Nitz NM, et al. Clinical detection of depression among communitybased elderly people with self-reported symptoms of depression. J Gerontol $A$ Biol Sci Med Sci 1998;53(2):M92-101.

206. Crawford MJ, Prince M, Menezes $P$, et al. The recognition and treatment of depression in older people in primary care. Int J Geriatr Psychiatry 1998;13(3):172-176.

207. Van Marwijk HWJ. Depressie bij ouderen in de huisartspraktijk. Tijdschr Gerontol Geriatr 1997;28:69-75.

208. Van Marwijk H, De Bock GH, De Jong JM, et al. Management of depression in elderly general practice patients. Scand J Prim Health Care 1994;12(3):162-168.

209. Bowers J, Jorm AF, Henderson S, et al. General practitioners' detection of depression and dementia in elderly patients. Med J Aust 1990;153(4):192-196.

210. Pond CD, Mant A, Bridges-Webb C, et al. Recognition of depression in the elderly: a comparison of general practitioner opinions and the Geriatric Depression Scale. Fam Pract 1990;7(3):190-194.

211. MacDonald AJ. Do general practitioners "miss" depression in elderly patients? Brit Med J (Clin Res Ed) 1986;292(6532):1365-1367.

212. Helmes E, Duggan GM. Assessment of depression in older adult males by general practitioners. Ageism, physical problems and treatment. Aust Fam Physician 2001;30(3):291-294 .

213. Wittchen HU, Hofler M, Meister W. Prevalence and recognition of depressive syndromes in German primary care settings: poorly recognized and treated? Int Clin Psychopharmacol 2001;16(3):121-135.

214. Hickie IB, Davenport TA, Scott EM, et al. Unmet need for recognition of common mental disorders in Australian general practice. Med J Aust 2001;175 Suppl:S18-24.

215. Kessler D, Lloyd $K$, Lewis $G$, et al. Cross sectional study of symptom attribution and recognition of depression and anxiety in primary care. Brit Med J 1999;318(7181):436-439.

216. Tylee A. Depression in the community: physician and patient perspective. J Clin Psychiatry 1999;60(Suppl 7):12-16; discussion 17-18.

217. Goldman LS, Nielsen NH, Champion HC. Awareness, diagnosis, and treatment of depression. J Gen Intern Med 1999;14(9):569-580.

218. Ventura J, Liberman RP, Green MF, et al. Training and quality assurance with the Structured Clinical Interview for DSM-IV (SCID-I/P). Psychiatry Res 1998;79(2):163-173.

219. Loor HI, Groenier KH, Schuling J, et al. Depressiviteit na een cerebrovasculair accident: het oordeel van de huisarts. Huisarts Wet 1999;42(7):299-302.

220. World Health Organization. International Statistical Classification of Diseases and Related Health Problems. 1989 Revision. Geneva: WHO; 1992.

221. Turner-Stokes L. Poststroke depression: getting the full picture. Lancet 2003;361(9371):1757-1758.

222. Morris PL, Shields RB, Hopwood MJ, et al. Are there two depressive syndromes after stroke? J Nerv Ment Dis 1994;182(4):230-234.

223. Betg $\mathrm{A}$, Palomaki $\mathrm{H}$, Lehtihalmes $\mathrm{M}$, et al. Poststroke depression: an 18-month follow-up. Stroke 2003;34(1):138-143.

224. Fazekas F, Barkhof F, Wahlund LO, et al. CT and MRI rating of white matter lesions. Cerebrovasc Dis 2002;13 (Suppl 2):31-36.

225. Goodman A. Organic unity theory: the mind-body problem revisited. Am J Psychiatry 1991;148(5):553-563. 
Cerebrovascular accidents (CVA or stroke) are a major health issue in the West. In the Netherlands, about 30,000 people suffer from stroke each year. At present, about 120,000 stroke survivors live with the consequences of stroke and are confronted with more or less severe disabilities and handicaps. Apart from the personal suffering that it causes, stroke costs society about 2 billion euro per year.

Depression is the most common psychiatric disorder after stroke. Post-stroke depression (PSD) is associated with longer duration of hospital admissions, less effect of rehabilitation programmes and a higher risk of dying within a few years after stroke. This makes PSD a clinically relevant problem. From a scientific perspective, PSD offers an opportunity to study the effect of focal brain damage on the regulation of mood.

During the last twenty years, a lot of research has focused on the hypothesis that stroke located in the left hemisphere is more strongly associated with depression than right-hemisphere stroke. The further towards the frontal pole the lesion is located the stronger this would be. As a consequence, PSD has been postulated as an organic psychosyndrome and the role of psychological and social factors in its pathogenesis have been relatively neglected.

This thesis is the result of cooperation between the Departments of Neurology and Psychiatry \& Neuropsychology of the University of Maastricht. It aims to contribute to the correction of this tendency by studying the role of both strokerelated and other factors on the development of PSD during the first year after stroke within one cohort of stroke patients. For this purpose, 190 consecutive stroke patients who were admitted to the University hospital of Maastricht were assessed for several potential risk factors for PSD. Depression was assessed on five different occasions during the first year after stroke, using self-assessment questionnaires and a structured diagnostic psychiatric interview. These risk factors consist of stroke-specific factors (lesion location, type of stroke, generalised vascular damage, etcetera) and non-stroke- specific factors (family history of depression, personal history of depression, personality characteristics, level of handicap, etcetera).

The choice for this design was supported by the outcome of literature reviews of the incidence of PSD and meta-analyses that re-analysed all available results on the effect of lesion location. It was concluded from these (systematic) reviews that many research findings on PSD are inconsistent and that there is no evidence to support the lesion location hypothesis as the only explanation for PSD. 
In chapter 2 of this thesis, several factors that may be responsible for the inconsistent findings from the different studies on PSD, are critically reviewed. In doing so, difficulties in diagnosing depression in stroke patients are discussed, as are differences between studies in research design, methods, and setting. Moreover, it is attempted to give an overview of published prevalence rates by regarding the different results against the background of some major differences in study characteristics. This approach reveals that higher prevalence estimations depend on a shorter period of time between stroke and the measurement of depression, on more specialised study settings (hospital, rehabilitation clinic), and on the use of self-report scales for depression rather than structured diagnostic instruments.

Finally, the two main biological hypotheses between vascular cerebral damage and depression are discussed.

In chapter 3 , four different, widely used rating scales for depression are studied for their validity as screening instruments for PSD. These are the Hospital Anxiety and Depression Scale (HADS), the Beck Depression Inventory (BDI), the 90-item Symptom Checklist (SCL-90), and the Hamilton Depression Rating Scale (HAMD). The predictive validity is determined by relating the scale scores on the different instruments to the outcome of a structured diagnostic psychiatric interview that allows for a diagnosis of major or minor depression according to DSM-IV (gold standard). Using so-called ROC-curves it is visualised which score on each rating scale is the optimum cut-off point to predict a depressive outcome with the highest possible accuracy. It is concluded that all four rating scales have acceptable screening abilities with only minor differences between the different instruments. At its optimum cut-off level, the HAMD was less sensitive in detecting post-stroke depression. However, there were fewer false positives in non-depressed patients. Moreover, the instruments were more accurate in men than women.

In chapter 4, it is described how often depression (defined as either 'major' or 'minor' depression according to DSM-IV) occurs in the first year after stroke, and how this relates to patients that suffered from a myocardial infarction (MI). It is interesting to compare these two patient groups because both have been subjected to an acute, life-threatening, vascular disease, which can result in functional disabilities and social handicaps. However, both groups differ in the fact that in the case of stroke but not MI, the brain is damaged itself.

For this study, data was used from the PhD study of Dr. J. Strik (dissertation: Mind the Heart, 2003), in which a cohort of about 200 MI patients was followed using the same study design and methods.

From the comparison between both groups, it showed that post-stroke depression occurred in almost $40 \%$ of patients as compared to almost $30 \%$ of MI patients. However, this difference was shown to rely on differences in sex, age, and level of 
handicap between both patient groups. Thus, although at first sight depression is more common in stroke than MI patients, this does not support the hypothesis that post-stroke depression is caused by the direct damage of brain areas that are involved in mood regulation.

In chapter 5, the five dimensions of the so-called Five Factor Model (FFM or Big Five) of personality are studied as vulnerability factors for PSD. The FFM distinguishes the dimensions Neuroticism, Extraversion, Openness (to new experiences), Agreeableness, and Conscientiousness. A high level of neuroticism, defined as the tendency to react to distressing circumstances by the experience of negative affects, and a low level of extraversion (also referred to as introversion), that describes the tendency of an individual to be turned towards one's inner world, have frequently been linked to a susceptibility to depression. These factors have hardly been studied in PSD research. In the study described in this dissertation, patients were asked to fill out the NEO-FFI, a self-rating questionnaire based on the FFM, within the first month after stroke. Multivariate Cox regression analysis revealed that neuroticism is the only independent predictor of PSD. The group with the highest neuroticism scores had a four to five times higher chance of developing PSD during the first year after stroke than did the group with the lowest scores. This effect was also significant if potential confounding factors, such as sex, age, level of handicap, and the level of psychological distress (measured by the SCL-90 total score), were taken into account. The level of handicap was the only other independent predictor of depression.

In chapter 6, the lesion location hypothesis and vascular depression hypothesis are tested in the same patient cohort, using data from clinical neurological assessments and imaging diagnostic techniques (CT and MR scans). The clinical data could be extracted from the so-called Maastricht Stroke Register, a large databank, in which data from all patients who were diagnosed with stroke at the University Hospital Maastricht are stored.

The lesion location hypothesis suggests that left-hemisphere stroke causes depression more frequently than right hemisphere stroke, especially when the lesion is located more to the proximity of the frontal pole. The vascular depression hypothesis supposes that generalised vascular damage in the brain, as apparent from white matter lesions and/or 'silent' (asymptomatic) infarcts on CT/MR scans, increase the vulnerability for depression.

Both separate and combined analyses did not reveal support for each of these hypotheses. However, neuroticism and level of handicap, which were added to the multivariate analysis as potential confounders, remained as significant independent risk factors. 
In chapter 7 , general and stroke-related risk factors were analysed according to a two-step procedure. This analysis strategy has been applied before in research into depression in Parkinson's disease patients and is restricted to the study of major depression (dissertation: Parkinson's disease, depression and serotonin, 2002 by Dr. A.F.G. Leentjens).

Firstly, a model of generally accepted risk factors of major depression in the community is tested for its explanatory strength in stroke patients. This model consists of the factors age, sex, personal history of depression, family history of depression, and somatic comorbidity (apart from stroke). Subsequently, strokerelated factors were added one by one and tested for their independent additive effect. These factors were level of handicap, cognitive functioning (MMSE score), hemisphere lesion location, frontal region involvement, and generalised vascular damage.

Using Cox regression analysis, the general risk factor model was shown to be significant. Within this model, personal history of depression, family history of depression, and (if 1-tailed level of significance was used) female sex were independent risk factors. From the stroke-related risk factors only the level of handicap turned out to add significantly to the model. Apart from the cerebrovascular factors (lesion location, generalised vascular damage), global cognitive functioning was also not found to be an independent risk factor for PSD.

In chapter 8 , a substudy is presented about the recognition and treatment of poststroke depression in general practice. This study is a reaction to a publication of Loor et al., who presented a good correlation between the outcome on depression self-rating scales and the recognition of PSD by GPs. This finding contrasts with several reports about underdiagnosis and undertreatment of depression in the elderly population as a whole.

In this PhD study, GPs of 58 patients from the cohort study (29 depressed and 29 non-depressed patients) were asked to participate in a telephone interview after the 1-year follow-up period of the cohort study was finished. An inventory was made of the number of patient contacts, the reasons for these contacts, attention to complaints and problems of a potentially psychiatric nature, the diagnosis that was made, as well as the suggested treatment.

The results show that depression, as defined according to the DSM-IV criteria of major or minor depression, is diagnosed by GPs in a little less than $40 \%$ of the cases. One third of depressed patients were treated with an antidepressant drug, while a follow-up appointment was made in a similar number of cases. Adequate recognition of depression by GPs was complicated to a large extent, because patients tended not to visit their GP with depressive complaints. In addition, minor depression and depression which was shown to be of shorter duration during the 1-year follow up, was recognised less often by the GP. The results indicate that 
there is room for improvement in the role of GPs in the management of poststroke patients.

In chapter 9, there follows a general discussion about the results presented in this dissertation. It is concluded that stroke increases the incidence of both major and minor depression but that this cannot be satisfactorily explained from a purely neurobiological perspective.

Supported by the findings summarised above, a biopsychosocial approach is needed to better understand the interplay between causal factors. In this perspective, stroke should be considered as an acute, life-threatening event with consequences at biological, psychological and social level. The severity of these distressing consequences determine, in combination with one's susceptibility to depression, if PSD will or will not occur.

To gain more consistent results from future studies, the comparability of research designs and methods should be optimised. Moreover, consensus should be reached about strict diagnostic criteria for PSD. On the one hand, large, longitudinal studies are needed to test multifactorial models for the independent effects of individual factors and possible interaction effects. On the other hand, newly gained insights about the cerebral organisation of mood from basic neurosciences must be adopted to innovate research into the relationship between stroke and mood (dys)regulation.

Finally, stroke patients would be helped by a better recognition and diagnostics of PSD in everyday clinical practice. For this purpose, stroke patients, their carers, and their professional caregivers should be better educated about behavioural disorders after stroke. Treatment should aim at the specific set of risk factors that are of importance in the individual patient. Thus, in one patient, pharmacological treatment may be more appropriate, while in others improvement of the social support system may deserve specific attention. 


\section{Samenvatting}

Cerebrovasculaire accidenten (CVA of beroerte) vormen een belangrijk gezondheidsprobleem in de Westerse landen. In Nederland worden jaarlijks circa 30.000 mensen door een beroerte getroffen en momenteel leven zo'n 120.000 mensen met de gevolgen ervan, hetgeen in meerdere of mindere mate gepaard gaat met beperkingen en handicaps. Dit veroorzaakt niet alleen persoonlijk lijden maar kost de maatschappij als geheel ongeveer 2 miljard euro per jaar.

Depressie is de meest voorkomende psychiatrische stoornis na een CVA. Depressie bij CVA patiënten gaat niet alleen gepaard met persoonlijk lijden maar wordt ook in verband gebracht met langer durende ziekenhuis opnames, minder effect van revalidatie programma's en een grotere kans op vroegtijdig overlijden in de jaren na de beroette. Dit maakt post-CVA depressie een klinisch relevant probleem. Vanuit wetenschappelijk perspectief biedt het bovendien de mogelijkheid het effect van focale hersenschade op de stemmingsregulatie te onderzoeken.

In de afgelopen 20 jaar is veel wetenschappelijke belangstelling uitgegaan naar de door onderzoeksresultaten gesteunde veronderstelling dat CVA's in de linkerhemisfeer vaker gepaard gaan met depressiviteit dan die in de rechterhemisfeer, vooral naarmate het CVA meer in de buurt van de voorhoofdskwab gelokaliseerd is. Hierdoor is post-CVA depressie gepostuleerd als een vorm van depressie met een organische oorzaak en is de rol van psychologische en sociale factoren lange tijd onderbelicht gebleven.

Dit proefschrift, dat een samenwerkingsproject is tussen de Capaciteitsgroepen Neurologie en Psychiatrie \& Neuropsychologie van de Universiteit Maastricht, beoogt bij te dragen aan een correctie van deze tendens door binnen een en dezelfde groep van CVA patiënten niet alleen CVA-gerelateerde maar ook andere factoren te bestuderen op hun bijdrage aan het ontstaan van depressie in het eerste jaar na een CVA. Hiervoor werden bij 190 opeenvolgende patiënten die met een CVA in het Academisch Ziekenhuis Maastricht werden onderzocht, diverse potentiële risicofactoren in kaart gebracht en werd vervolgens gedurende 1 jaar op vijf verschillende meetmomenten depressie gemeten met behulp van vragenlijsten en een gestructureerd diagnostisch psychiatrisch interview. Deze risicofactoren omvatten CVA-specifieke factoren (lokatic van het CVA, type CVA, gegeneraliseerde vaatschade, etcetera) en niet-CVA-specifieke factoren (familiair voorkomen, depressie in de voorgeschiedenis, persoonlijkheidskenmerken, mate van handicap, etcetera).

De keuze voor deze opzet werd ondersteund door recente overzichtsartikelen die aantoonden dat de verschillende onderzoeken naar het voorkomen van post-CVA depressie en naar de rol van laesielokatie onvoldoende consistente resultaten 
hadden opgeleverd om de veronderstelde relatie als enige verklaring te blijven verdedigen.

In hoofdstuk 2 van dit proefschrift wordt een kritische beschouwing gegeven van de diverse factoren die ten grondslag (kunnen) liggen aan de inconsistente bevindingen die de verschillende onderzoeken naar post-CVA depressie hebben opgeleverd. Hierbij wordt ingegaan op moeilijkheden bij de diagnostiek van depressie bij CVA patiënten en op verschillen tussen studies in onderzoeksdesign, onderzoeksmethoden en onderzoekssetting. Tevens wordt een overzicht gegeven van gepubliceerde prevalentie cijfers, waarbij aan de hand van onderverdeling naar een aantal studiekarakteristieken gepoogd wordt ordening aan te brengen in de uiteenlopende prevalentie getallen. Hieruit blijkt dat hogere prevalentieschattingen gerelateerd zijn aan een kortere tijdsperiode tussen het CVA en het meten van depressie, aan meer gespecialiseerde onderzoekssettingen (onderzoek in ziekenhuizen en/of revalidatiecentra versus bevolkingsonderzoek) en aan het gebruik van zelfrapportage schalen voor depressie in plaats van gestructureerde diagnostische instrumenten.

Ten slotte worden de twee voornaamste biologische hypotheses over de relatie tussen vasculaire cerebrale schade en depressie besproken.

In hoofdstuk 3 worden 4 verschillende, veelgebruikte meetinstrumenten voor depressie bestudeerd op hun bruikbaarheid als screeningsinstrumenten voor postCVA depressie. Dit betreft de Hospital Anxiety and Depression Scale (HADS), de Beck Depression Inventory (BDI), de Symptom Checklist-90 (SCL-90), en de Hamilton Depression Rating Scale (HAMD). De predictieve validiteit wordt bepaald door scores op de verschillende meetschalen te relateren aan de uitkomst van een gestructureerd diagnostisch psychiatrisch onderzoek dat resulteert in een diagnose van ('major' of 'minor') depressie volgens DSM-IV (gouden standaard). Aan de hand van zogenaamde ROC-curves wordt zichtbaar gemaakt welke score op elke meetschaal het beste afkappunt vormt om het bestaan van post-CVA depressie het beste te voorspellen en met welke accuratesse. Uit de analyses bleken alle vier de instrumenten acceptabele screeningskwaliteiten te hebben en er slechts kleine verschillen tussen de verschillende meetschalen te bestaan. De HAMD was bij zijn optimale afkappunt minder sensitief in het detecteren van depressie maar scoorde daarentegen minder vaak ten onrechte positief bij niet-depressieve patiënten, wijzend op een hogere specificiteit. De instrumenten presteerden overigens duidelijk beter bij mannen dan bij vrouwen.

In hoofdstuk 4 wordt beschreven hoe vaak depressie (gedefinieerd als of 'major' of 'minor' depressie volgens DSM-IV) in het eerste jaar na een beroerte optreedt en hoe zich dit verhoudt tot het voorkomen van depressie bij patiënten die een 
hartinfarct hebben doorgemaakt. De vergelijking tussen deze twee patiëntengroepen is interessant omdat beide getroffen zijn door een acute, levensbedreigende vasculaire aandoening die kan resulteren in functionele beperkingen en sociaal-maatschappelijke handicaps. Beide groepen verschillen echter in het feit dat in geval van een beroerte maar niet van een hartinfarct de hersenen zelf worden beschadigd.

Voor dit onderzoek werd gebruik gemaakt van gegevens uit het promotieonderzoek van Dr. J. Strik (dissertatie Mind the Heart, 2003), waarin een cohort van circa 200 hartinfarct patiënten volgens dezelfde onderzoeksopzet en methoden werden gevolgd.

Uit de vergelijking tussen beide groepen bleek depressie na een CVA in bijna $40 \%$ voor te komen en na een hartinfarct in bijna 30\%. Aan dit verschil bleken echter verschillen in leeftijd, geslacht en de mate van handicap tussen de CVA en hartinfarct patiënten ten grondslag te liggen. Dus, hoewel op het eerste gezicht depressie inderdaad vaker lijkt voor te komen na een herseninfarct dan na een hartinfarct, vormt dit geen ondersteuning voor de hypothese dat post-CVA depressie veroorzaakt wordt door de directe beschadiging van hersengebieden die bij de regulatie van de stemming zijn betrokken.

In hoofdstuk 5 worden de vijf dimensies uit het zogenaamde Five Factor Model (FFM of Vijf Factoren Model) van persoonlijkheid bestudeerd als kwetsbaarheidsfactoren voor post-CVA depressie. De FFM onderscheidt de dimensies Neuroticisme, Extraversie, Openheid (voor nieuwe ervaringen), Altruisme en Consciëntieusheid. Hoge mate van neuroticisme, dat wordt gedefinieerd als de neiging op stresserende omstandigheden te reageren met het ervaren van negatieve affecten, en lage mate van extraversie (ook wel introversie genoemd), dat de neiging van het individu beschrijft om gericht te zijn op zijn/haar binnenwereld, zijn veelvuldig in verband gebracht met kwetsbaarheid voor depressie. In het onderzoek naar post-CVA depressie zijn persoonlijkheidsfactoren vrijwel niet bestudeerd. Patiënten in het hier beschreven promotieonderzoek werden gevraagd om in de eerste maand na het CVA de NEO-FFI, een zelfbeoordelingsvragenlijst naar de vijf persoonlijkheidsdimensies uit het FFM, in te vullen. Uit multivariate Cox regressie analyse bleek neuroticisme de enige onafhankelijke voorspeller te zijn van post-CVA depressie, waarbij de groep met de hoogste scores een 4 tot 5 maal zo grote kans hadden om depressie te ontwikkelen in het eerste jaar na de beroerte dan de groep met de laagste scores. Dit effect bleef bestaan ook als werd gecontroleerd voor mogelijke verstorende factoren, zoals leeftijd, geslacht en mate van handicap en voor de mate van psychologische stress die patiënten in de week van invullen van de vragenlijst ervoeren (gemeten met de SCL-90). 
De enige andere onafhankelijke voorspeller van depressie was de mate van handicap, waarmee patiënten zich na het CVA geconfronteerd zagen.

De resultaten vormen een belangrijke ondersteuning voor de hypothese dat postCVA depressie niet (uitsluitend) berust op neurobiologische schade maar dat de wijze waarop een patiënt vanuit zijn persoonlijkheid reageert op de beroerte (mate van neuroticisme) en de mate waatin hij door de beroerte wordt getroffen in zijn/haar sociaal-maatschappelijke functioneren (mate van handicap) tevens bepalend zijn.

In hoofdstuk 6 worden in dezelfde cohort studie de laesie locatie hypothese en vasculaire depressie hypothese getoetst aan de hand van data uit klinisch neurologisch onderzoek en beeldvormende diagnostische technieken (CT en MRI scans). De klinische gegevens konden worden geëxtraheerd uit het zogenaamde 'Maastricht Stroke Register', een omvangrijke databank, waarin gegevens over alle CVA patiënten die in het Academisch Ziekenhuis Maastricht worden gezien, zijn opgeslagen.

De laesie locatie hypothese stelt dat linkszijdige CVA's vaker gepaard gaan met depressie dan rechtszijdige CVA's, vooral naarmate de laesie meer in de buurt van de voorhoofdskwab gelokaliseerd is. De vasculaire depressie hypothese veronderstelt dat gegeneraliseerde vaatschade in de hersenen, zoals blijkt uit witte stof afwijkingen en/of 'stille' (symptoomloze) infarcten op CT/MRI scans, de kwetsbaarheid voor depressie vergroot.

Zowel in separate als gecombineerde analyses is voor beide hypotheses in het hier beschreven onderzoek geen ondersteuning gevonden. Wel blijven neuroticisme en handicap, die als mogeliik verstorende variabelen aan de analyse werden toegevoegd, als onafhankelijke risicofactoren overeind.

In hoofdstuk 7 worden algemene en CVA-gerelateerde risicofactoren (waaronder diverse factoren die hierboven reeds werden beschreven) geanalyseerd volgens een 2 stappen procedure. Deze analysestrategie werd eerder toegepast in onderzoek naar depressie bij Parkinson patiënten en beperkt zich tot het bestuderen van 'major' depressie (proefschrift Parkinson's disease, depression and serotonin, Dr. A. Leentjens).

Eerst wordt een model van algemeen erkende risicofactoren van 'major' depressie in de algemene bevolking getoetst op verklarende kracht bij CVA patiënten. Dit model bestaat uit de factoren leeftijd, geslacht, voorgeschiedenis van depressie, familie anamnese voor depressie en somatische co-morbiditeit (naast het bekende CVA). Daarna werden CVA-gerelateerde factoren een voor een aan dit model toegevoegd en getoetst op hun onafhankelijke, additionele effect. Deze factoren betroffen mate van handicap, cognitief functioneren (MMSE score), hemisfeer laesie locatie, voorhoofdskwab beschadiging en gegeneraliseerde vasculaire schade. 
Het algemene risicofactor model dat werd getoetst met behulp van Cox regressie, was als geheel significant, waarbij een positieve voorgeschiedenis van depressie, een positieve familie-anamnese en (indien eenzijdig getoetst) vrouwelijk geslacht onafhankelijke voorspellers waren. Van de CVA-gerelateerde factoren bleek alleen mate van handicap (opnieuw) als onafhankelijke, additionele risicofactor aan verbetering van het model bij te dragen. Naast de cerebrovasculaire factoren (laesie locatie, gegeneraliseerde vaatschade) bleek ook cognitief disfunctioneren in dit onderzoek geen risicofactor voor post-CVA depressie.

In hoofdstuk 8 wordt een deelonderzoek beschreven naar de herkenning en behandeling van post-CVA depressie in de huisartsenpraktijk. Dit onderzoek is een reactie op een publicatie van Loor en collega's die goede overeenstemming rapporteerden tussen de uitkomst van depressie zelfscorelijsten en het signaleren van post-CVA depressie door de huisartsen. Deze bevinding contrasteert met diverse publicaties over onderdiagnostiek en -behandeling van depressie bij ouderen in het algemeen.

In dit promotieonderzoek werd bij 58 patiënten uit de cohortstudie (29 depressieve en 29 niet-depressieve patiënten) de huisarts na afronding van de 1-jaars follow-up periode benaderd voor een telefonisch interview. Het aantal contacten met de patiënt gedurende het jaar na het CVA, de reden van de consulten, het bespreken van klachten / problemen van mogelijk psychi(atri)sche aard, de gestelde diagnose en het voorgestelde beleid werden geïnventariseerd.

De resultaten tonen dat depressie, zoals gedefinieerd aan de hand van de DSM-IV criteria voor major of minor depressie, door huisartsen slechts in iets minder dan $40 \%$ van de gevallen was geconstateerd. Van de depressieve patiënten werd een derde deel behandeld met een antidepressivum, terwijl met een even groot deel een controleafspraak gemaakt werd. Goede herkenning van depressie door de huisarts werd voor een belangrijk deel bemoeilijkt doordat patiënten neigden om niet met hun depressieve klachten naar de huisarts te gaan. Daarnaast werden minor depressies en depressies die gedurende de 1-jaars follow-up een kortere duur hadden vertoond minder vaak door de huisarts gedetecteerd. Het onderzoek toont aan dat er ruimte is voor verbetering van de signalexings- en zorgfunctie van huisartsen voor post-CVA patiënten.

In hoofdstuk 9 volgt tenslotte een algemene beschouwing over de in dit proefschrift gepresenteerde onderzoeksbevindingen. Concluderend wordt gesteld dat CVA de incidentie van zowel major als minor depressie doet stijgen maar dat dit onvoldoende verklaard kan worden vanuit puur neurobiologisch perspectief.

Gesteund door de hierboven samengevatte bevindingen is een biopsychosociale benadering nodig om het samenspel van oorzakelijke factoren beter te begrijpen. Een CVA moet in dit kader worden beschouwd als een acute, levensbedreigende 
gebeurtenis met gevolgen op biologisch, psychologisch en sociaal terrein. De ernst van deze stresserende gevolgen bepalen in combinatie met iemands kwetsbaarheid voor depressie of post-CVA depressie al dan niet ontstaat.

Om uit toekomstig onderzoek meer reproduceerbare resultaten te verkrijgen zouden de vergelijkbaarheid van onderzoeksdesigns en -methoden moeten worden verbeterd en zou consensus moeten worden bereikt over strikte diagnostische criteria voor post-CVA depressie. Enerzijds zijn grote longitudinale studies noodzakelijk om multifactoriële verklaringsmodellen te onderzoeken op de onafhankelijke bijdrage van afzonderlijke factoren en op de mogelijke interacties tussen hen. Anderzijds zouden nieuw verworven inzichten over de cerebrale organisatie van stemming uit de basale neurowetenschappen overgenomen moeten worden om de relatie tussen CVA en stemmings(dys)regulatie nieuwe impulsen te geven.

Tenslotte is de CVA patiënt gebaat bij een betere signalering en diagnostiek van post-CVA depressie in de dagelijkse klinische praktijk. Hiertoe zouden CVA patiënten, hun verzorgers en hun professionele hulpverleners beter geïnformeerd (respectievelijk bijgeschoold) moeten worden over gedragsstoornissen na een beroerte. De behandeling dient te worden afgestemd op de specifieke constellatie van risicofactoren die bij een individuele patiënt van betekenis zijn. Zo kan bij de ene patiënt medicamenteuze behandeling meer voor de hand liggen terwijl bij de ander versterking van het sociaal steunsysteem meer nadruk verdient. 


\section{Dankwoord}

Om na 7 jaar eindelijk te promoveren is een opluchting! Een succesvolle afsluiting van een proces dat niet zonder slag of stoot verlopen is. Het AIO-schap is niet alleen een voortzetting van mijn opleiding geworden maar in zekere zin ook een soort voortzetting van mijn opvoeding. Aan het begin van mijn 'wetenschappelijke carrière' bruiste ik van de jeugdige ambities en grote verwachtingen met een ongebreideld vertrouwen in mijn eigen kwaliteit. Ik had bovengemiddelde pretenties. $\mathrm{Nu}$ aan het einde van dit begin, ligt een bescheiden proefschrift voor mij als tastbare uitkomst van een proces van groei. Van groei naar het gemiddelde.

Hooggeleerde Prof. dr. Verhey, beste Frans, dank voor je dagelijkse begeleiding bij dit proces, dat meer tijd en inspanning heeft gevergd dan je had gehoopt. Ik geloof dat ik je vaker verbaasd heb achtergelaten na weer een uur overleg over de voortgang van het project. Maar ook omgekeerd heb jij mij verward met je combinatie van empathisch geduld en snelle dadendrang en met je betrokkenheid bij het wetenschappelijk proces in combinatie met een zeker zakelijk opportunisme. Je bent in mijn beleving uit verschillende soorten hout gesneden: dat van de psychiater, de wetenschapper, de zakenman en de diplomaat. Lang kon ik de ene beter waarderen dan de andere maar inmiddels zie ik dat ze alle even belangrijk waren om mij naar de eindstreep te loodsen. Daarvoor ben ik je nu erkentelijk. Tevens dank ik je voor je interesse en betrokkenheid bij de grote gebeurtenissen in mijn bestaan. Want de jaren van het AIO-schap waren tegelijk de jaren van mijn trouwen, van drie maal verhuizen, van driemaal vader worden van (in totaal) drie dochters maar ook van ziekte- en sterfgevallen in mijn (schoon)familie. Je herhaaldelijke vragen naar thuis tijdens die roerige fasen waren voor mij de beste momenten van ons wekelijkse uur werkbegeleiding.

Weledelzeergeleerde Dr. Honig, beste Adriaan, ook jou dank ik voor je volhardende inzet bij de begeleiding van mijn promotieproject, waarbij je de parallelle schakeling tussen het CVA en MI project als sterkste bewaakte. Ik geloof dat hoofdstuk 4 van dit proefschrift, dat eerder werd gepubliceerd in een vooraanstaand wetenschappelijk tijdschrift, daarvan een waardevol resultaat is. Onze samenwerking was echter een moeilijke. Ik denk door de wezenlijke verschillen in onze karakters maar waarschijnlijk ook wel door een aantal gelijkenissen. Voor mij soms een beproeving; waarschijnlijk ook voor jou. Maar inmiddels proef ik de verdienstelijke smaak van dit, bij eerste beet, bedenkelijke fruit. Zoals een grapefruit, die je als kind niet lust maar later eet in het betere restaurant. Want langzaam ontdek ik de bronnen van mijn eigen weerstand en 
verzet die ons bij het samenwerken parten speelden. Dat voelt als een verrijking, waarin jij een ondankbare maar wezenlijke rol hebt gespeeld. Hiervoor betuig ik je hierbij mijn erkentelijkheid.

Weledelzeergeleerde Dr. Lodder, beste Jan, dank voor het 'copromoten' gedurende bijna tweemaal een AIO termijn. Om je dan toch niet officieel als copromotor aan dit proefschrift verbonden te weten, voelt als een gemis. Je was voor mij, behalve neurologische vraagbaak, een baken bij mijn dwalen! Relativerend, de rust bewarend en niet te vergeten vol filosofisch voer uit het verre Oosten. De parabel over de leerling samurai is sterker op mij van toepassing gebleken dan ik bij eerste lezing gewenst had. En mooi om te zien dat de meester zelf het hout sprokkelen niet uit de weg ging (lees: stapels CT's en MRI's beoordelen)! Dank je wel, Jan. Als ook het laatste artikel nog wordt gepubliceerd, lijkt me dat ons reeds lang geplande etentje een gepaste afsluiting van onze samenwerking zou zijn. In jouw tuin of de mijne?

Het kan raar lopen: begonnen met drie copromotoren, eindig ik met 1 promotor en 1 copromotor. Onze gezamenlijke weg van 3 geleerden en hun leerling was meer te vergelijken met een afwisselende klim- en klautertocht in de Ardennen dan met een bezinnende wandeling door de rustieke tuinen van een oud College te Cambridge. Soms over smalle, rotsige paden door nat en mistig weer maar vaker ook over groene heuvels met weidse vergezichten bij helder zonnig weer.

Zonnig was het ononderbroken in de samenwerking met de collega's 'in het veld'.

Lieve Jacqueline, we delen inmiddels een hele geschiedenis. Samen studeren, samen promoveren en nu samen in opleiding tot psychiater. We zijn in de AIO tijd steeds dichter naar elkaar gegroeid en collegialiteit werd vriendschappelijkheid. We hebben gezwoegd, we hebben gelachen en bovenal hebben we heel wat gebeurtenissen in ons leven gedeeld. Jouw attente betrokkenheid bij die van mij heb ik altijd als steun ervaren. Sinds we de UM verruilden voor Vijverdal, zijn we elkaar een stuk uit het oog verloren. $\mathrm{Nu}$ het leven op vele fronten weer rustiger en overzichtelijker lijkt te worden, hoop ik dat we de opgebouwde band weer kunnen aantrekken.

Beste Richel, het was in vele opzichten een voorrecht om jou als kamergenoot in de buurt te hebben. Niet alleen dank ik een heel wezenlijk deel van wat ik aan wetenschappelijke kennis en kunde heb vergaard aan jou grote onderwijzende kwaliteiten maar vooral ook heb je mijn plezier in het doen van onderzoek weten vast te houden. Daarnaast hebben we heel wat beschouwende gesprekken gevoerd over andere dan wetenschappelijke thema's. Of en hoe die hebben bijgedragen aan 
het tot stand komen van dit proefschrift, durf ik niet goed te zeggen maar ik koester ze als richtingwijzers in mijn leven. Dank je wel, Richel!

Lieve Sascha, Pauline, Marjolein, Susan, Suzanne en Petra, voor mij vormden jullie samen met Jacqueline een bijzonder fijne club collega-AIO's 'van het eerste uur'. Als enige man in het gezelschap, voelde ik me vaak verwend. Thuis had ik het dan ook wel eens glimlachend over "mijn vrouwen." Samen met enkele anderen hebben we geprobeerd de gezamenlijkheid van het wetenschapsbedrijf 'Psychiatrie \& Neuropsychologie' op de werkvloer te bevorderen door in een informeel overleg elkaars kennis en ervaring te delen. Maar vooral ook door de gezellige avondjes en etentjes! Marjolein, jou ben ik speciale dank verschuldigd. Portofino 2000, heeft goede, rijpe wijn geleverd! Ik hoop dat we elkaar blijven ontmoeten.

Lieve vrienden van buiten de universiteit, Bas, Christian, Frank, Fred, Rob en Vincent, dat ik jullie nog vrienden durf te noemen, zegt vooral iets over jullie trouw naar mij. Want in de afgelopen jaren hebben we bar weinig vriendschap 'geconsumeerd'. Ik doe geen beloftes meer maar zonder goede voornemens is nog nooit iemand van het roken afgekomen... Bas, mijn beste vriend, al ben ik 15 jaar geleden van de ene op de andere dag naar Maastricht vertrokken, het gevoel van je nabijheid is altijd gebleven. Vanzelfsprekend wens ik jou achter mij bij het verdedigen van het proefschrift. Christian, we hebben heel wat met elkaar gemeen: een boel karaktertrekken, een gezin met kleine kinderen, onze studententijd en nu ook een promotie! Ik ben trots ook jou als paranimf achter me te weten!

Alle mensen die betrokken zijn geweest bij de uitvoering van mijn promotiestudie bedank ik voor hun inspanningen. In de eerste plaats de ondetzoeksassistentes Inge Crolla, Kirsten Venrooij, Patricia Hameleers, Manon Hanssen, Miriam Dietvorst en Marlies van Duinen. Patiënten-interviews plannen, NPO's afnemen, vragenlijsten verzenden, data invoeren, huisartsen interviewen, enzovoorts. Jullie hebben vele uren consciëntieus werk geleverd en altijd met een goed humeur. Het spijt mij dat veel van jullie werk nog niet eens in het uiteindelijke proefschrift is beland.

Anita Boreas, die als zogenaamde AGIKO tegelijk promoveerde en zich specialiseerde in de Neurologie. Je hebt me veel werk bespaard door dagelijks te registreren welke patiënten er met een CVA in het AZM waren opgenomen en door het bijhouden van het Maastrichtse Stroke Register. De stagiaires Ernst Brand, Lahcen Hahni, Susanne van Tuijl en Ralf Vrösch die zich hebben ingezet voor verschillende onderdelen van het promotieproject en met hun enthousiasme en leergierigheid mij weer enthousiaster maakten. De co-auteurs, Johan Denollet, George Beusmans, Albert Leentjens en Prof. Maes dank ik voor hun waardevolle bijdragen aan de artikelen. Johan, het was een verrijking om samen met je te schrijven aan het artikel over persoonlijkheid. Niet alleen kun je jouw rijke kennis 
op dat terrein heel helder overdragen maar ook werkt je enthousiaste en steunende houding heel bekrachtigend. Publicatie in Stroke was er zonder jou niet van gekomen. Albert, je hebt in korte tijd mijn proefschrift met een extra hoofdstuk meer inhoud gegeven. Ik hoop onder jouw supervisie op de polikliniek Eetstoornissen me verder te verbeteren in effectiviteit en efficiëntie. George, door je positivisme is uit een op het eerste oog problematisch conflict van belangen tot een gemeenschappelijk artikel geworden! Daarnaast heb ik je oprechte interesse in mijn persoonlijke omstandigheden steeds als zeer steunend ervaren. Professor Maes, onze samenwerking was slechts kort maar ik heb onze gedachtewisseling over de mogelijke relatie tussen uw onderzoeksterrein en het thema van dit proefschrift als heel inspirerend ervaren.

Het secretariaat van de staf Psychiatrie, met name Kitty Verwoerdt, en het secretariaat van de polikliniek dank ik voor het geduld met mijn eeuwige lastminute (and far beyond...) werk. Kitty, bij jou vond ik steeds een veilige haven als ik even voor de 'bazen' wilde schuilen.

Jane Sykes, Sophie Duijn en Linda Nijsters dank ik voor het corrigeren van mijn Engels. Lieve Sophie het was fijn om op jouw 'native knowledge of the English language' te mogen terugvallen toen ik weer eens in tijdnood zat. Laten we snel weer eens vieren dat we (in jullie referentiekader) bijna buren zijn.

De afdeling Neurologie van het AZM (C5), afdeling 5 van Revalidatiecentrum Hoensbroek en de verpleegklinieken Klevarie en De Zevenbronnen waar ik vele onderzoekspatiënten tijdens het jaar van follow-up bezocht, dank ik voor hun gastvrijheid en behulpzaamheid. Daarbij denk ik ook aan de klinische en ambulante zorgcoördinatoren van het Transmuraal Zorg Project, Francien Onstenk en Herbert Habets.

Mijn werkbegeleiders Jean-Paul Glaser, Marcel Hilwig en Carsten Leue van de Psychiatrie opleiding, dank voor jullie begrip voor mijn dubbele agenda. Jullie flexibiliteit om mij te helpen tijd te vinden om het proefschrift uiteindelijk toch eens af te ronden, heb ik zeer nodig gehad. Mijn supervisor van de Psychiatrie opleiding, Rob Havermans, bedankt Rob, voor je begripvolle, luisterende oor en voor je goede raad bij het omgaan met mijn promotieperikelen.

Lieve mama, bedankt voor de onvoorwaardelijke steun die u en papa mij altijd gegeven hebben bij het maken van keuzes voor mijn toekomst. Ook al moest u aan mijn keuze voor de Psychiatrie wel even wennen. Weet dat ik dankbaar ben voor de stevige fundamenten waarop mijn huidige bestaan is gebouwd. Ik weet dat papa trots zou zijn. Ik zal hem missen op de dag van mijn promotie.

Lieve Sigrid, lieve Merel, Fleur en Marjolein, jullie dank ik dat dit proefschrift ertoe doet, dat ik ertoe doe. Wat het meeste nabij is, voelt al snel vanzelfsprekend. Heel wat privé uren die voor jullie waren bedoeld en die jullie nodig hadden, zijn 
geofferd aan dit proefschrift. Ik weet wat dat gekost heeft. Dank jullie wel. En nu konijntjes kopen!

Lieve, Audrey en Deborah, mijn grote nichten, jullie horen bij ons gezin en waren fantastisch als hulp voor Sigrid en als speelkameraadjes voor de kinderen. Komend jaar zijn jullie zelf aan de beurt om een diploma te halen. Zet hem op!

Als laatste maar niet als minste dank ik alle onderzoekspatiënten, die zoveel moeite hebben gedaan om na de ingrijpende tegenslag van een CVA zich in te zetten voor wat De Wetenschap heet. We hebben veel van u gevraagd. Dit boekje is dan ook voor $u$ en voor degenen die hetzelfde lot trof of zal treffen. 


\section{Curriculum vitae}

Ivo Aben werd geboren op 8 April 1971 in Hoorn. In 1989 behaalde hij zijn VWO diploma aan de 'S.G. Werenfridus' in Hoorn en startte met de studie Geneeskunde aan de Universiteit Maastricht. Tijdens zijn studie werkte hij in aansluiting op een wetenschapsstage als student-assistent aan de vakgroep Geestelijke Gezondheidskunde (GGK). Na zijn doctoraal examen volgde hij gedurende ruim een jaar een drietal stages in het buitenland. Drie maanden klinische neurologie in het Klinikum te Aken, gevolgd door vier maanden psychiatrie aan de Universiteit van Cambridge en negen maanden neuropathologie aan de Universiteit van Wenen. In 1997 behaalde hij zijn artsendiploma en startte als AIO aan de Vakgroep Psychiatrie en Neuropsychologie van de Universiteit Maastricht. Sinds april 2001, is hij in opleiding tot psychiater verbonden aan PMS Vijverdal te Maastricht. In het kader hiervan werkte hij gedurende een jaar op de gesloten opname afdeling van PMS Vijverdal en een jaar voor het transmurale zorgproject Psycope. Thans werkt hij op de MedPsychUnit van het Academisch Ziekenhuis te Maastricht. In oktober 2005 hoopt hij zijn opleiding af te ronden. 